SCR-663

Sindia Corporation

\title{
MASTER
}

•...... MONOGRAPH

\section{TRANSIENTS IN WIDE-ANGLE CONICAL ANTENNAS}

by

C W Harrison, Jr and

C S Williams, Jr

JUNE 1963 


\section{DISCLAIMER}

This report was prepared as an account of work sponsored by an agency of the United States Government. Neither the United States Government nor any agency Thereof, nor any of their employees, makes any warranty, express or implied, or assumes any legal liability or responsibility for the accuracy, completeness, or usefulness of any information, apparatus, product, or process disclosed, or represents that its use would not infringe privately owned rights. Reference herein to any specific commercial product, process, or service by trade name, trademark, manufacturer, or otherwise does not necessarily constitute or imply its endorsement, recommendation, or favoring by the United States Government or any agency thereof. The views and opinions of authors expressed herein do not necessarily state or reflect those of the United States Government or any agency thereof. 


\section{DISCLAIMER}

Portions of this document may be illegible in electronic image products. Images are produced from the best available original document. 
Published by

Sandia Corporation,

a prime contractor to the

United States Atomic Energy Commission

LEGAL NOTICE

This report was prepared as an account of covernment sponsored work,
Neither the United States, nor the Commission, nor any person actios on behal of the Commission:

A. Makes any warranty or representation, expressed or implied, with respect to the ars disclosed in this report may not infringe privately owned rights; or

B. Assumes any liablitites with respect to the use of, or for damages resulting trom the use of any

As used in the above, "person setting on behatf of the Commission" inctudes
employee or contractor of the Commission, or employee of such contractor to the extent that contretor of the Conmission, or employee of such contractor, such sonsratior prepares, disseminates, or provides acosess to, any information pursuant to his employment or contract with the commission, or his employment 
SANDIA CORPORATION MONOGRAPH

TRANSIENTS IN WIDE-ANGLE CONICAL ANTENNAS

by

C. W. Harrison, Jr.

and

C. S. Williams, Jr.

June 1963

R 


\section{ACKNOWLEDGMENT}

The writers thank Prof. R. W. P. King and

Dr. Hans J. Schmitt for reading the paper. Margaret

Houston prepared the graphs.

The response of an antenna to DC pulse excitation, both in reception and in transmission, can be determined by use of Fourier transforms provided the impedance and effective height of the antenna are known over the frequency range where the excitation has significant components. We have computed the time history of the response of a wide-angle conical antenna to DC pulse excitation and show the results herein for a matched source in transmission and a matched load in reception for several values of cone angle and antenna length. Under matched conditions, the faithful transmission of a pulse shape requires a long antenna while faithful reception is not possible. Faithful reception can be achieved by a short antenna with a resistive load of high resistance or with a capacitive load, or by a long antenna with a series RC load. Since this work required impedance and effective height of conical antennas over a wide frequency range, we have also presented here graphs of these quantities for a number of cone angles. 
2. Précis . . . . . . . . . . . . . . . . . . . . . . . . . . . 6

2.1 Transmission . . . . . . . . . . . . . . . . . . . . . . . . . . . . . . . . . . 6

2.2 Reception . . . . . . . . . . . . . . . . . . . . . . . . . . . . . . 6

3. Analysis . . . . . . . . . . . . . . . . . . . . . . . . . . . . . . . . 7

3.1 Driving-Point Impedance . . . . . . . . . . . . . . . . . . . . . . . . . . . . . 7

3.2 Effective Length . . . . . . . . . . . . . . . . . . . . . . . . . . . . . . 9

3.3 Impedance and Effective T ength of a Shnrt, Wide-Angle Conioal Antcnna . . . . . . . 11

3. 4 Impedance and Effective Length of a Long, Wide-Angle Conical Antenna . . . . . . . 12

3.5 Numerical Results for Impedance and Effective Length . . . . . . . . . . . . . . . 13

3.6 Transfer Function of Antenna and Source in Transmission . . . . . . . . . . . . . 13

3.7 Time History of the Radiated Field . . . . . . . . . . . . . . . . . . . . . . . . . 14

3. 8 Transfer Function of Antenna and Load in Reception . . . . . . . . . . . . . . . . . 14

3.9 Time History of Load Voltage . . . . . . . . . . . . . . . . . . . . . . . . . . . . 15

3.10 Transmission Transfer Function for Short Antenna With Matched Source . . . . . . . 15

3.11 Transmission Transfer Tunction for Long Antenna With Matched Source . . . . . . . 16

3.12 Reception Transfer Function for Short Antenna With Matched Load . . . . . . . . . 16

3.13 Reception Transfer Function for Long Antenna With Matched Load . . . . . . . . . 17

3.14 Reception Characteristic of Long Antenna With Differentiating-Circuit Load . . . . . 17

3.15 Reflected Voltage on the Line . . . . . . . . . . . . . . . . . . . . . . . . . 18

3.16 Remarks. . . . . . . . . . . . . . . . . . . . . . . . . . 18

4. Appendix . . . . . . . . . . . . . . . . . . . . . . . . . . . . . . 20

4.1 Frequency-Domain Representation of a Time Function . . . . . . . . . . . . . . . 20

4.2 Transfer Function of a Linear System . . . . . . . . . . . . . . . . . . . . . . . 21

4. 3 Time Differentiation and Integration . . . . . . . . . . . . . . . . . . . . . . . 22

4.4 Reduction of the Number of Parameters . . . . . . . . . . . . . . . . . . . . . . . . 23

4.5 Excitation Pulses . . . . . . . . . . . . . . . . . . . . . . . . . . . . . 25

4. 6 Limiting Value of $\beta / \alpha$. . . . . . . . . . . . . . . . . . . . . . . . . . . . . 26

4. 7 Effective Length of Long Antenna . . . . . . . . . . . . . . . . . . . . . . . . . 28

LIST OF REFERENCES . . . . . . . . . . . . . . . . . . . . . . . . . . . . . . . 64 


\section{LIST OF ILLUSTRATIONS}

Figure

1

2

3

$4,5,6$, 7 , and 8

9,10 and 11

$12-21$

$22-31$

$32 a, b$, $33 a, b$, and $34 a$, b,

$35 a, b$, $36 a, b$, and $37 a, b$,
Page

Spherically-Capped, Conical Antenna Fed by Coaxial Feed Line . . . . . . . . . . 30

Equivalent Circuits for Transmission and Reception . . . . . . . . . . . . . 31

The Two Excitation-Pulse Shapes . . . . . . . . . . . . . . . . . 32

The Time History of the Radiated Field in the Equatorial Plane at 1000 Meters From The Antenna, Translated $r / c=10^{-5} / 3 \mathrm{sec}$ to Left to Remove Delay due to 1000 Meters of Travel . . . . . . . . . . . . . . . . .

The Time History of Voltage at Receiving Antenna Terminals. (Ordinate is Negative of Load Voltage Divided by Pulse Width. ) . . . . . . . . . . . . . . . . . 35, 36

Input Impedance $Z_{0}=R_{0}+j x_{0}$ of Wide-Angle Conical Antennas . . . . . . . . . 37-46

Radian Effective Length $\mathrm{kh}_{\mathbf{e}}=\mathrm{k}\left[\ddot{h}_{\mathbf{e}}+\mathrm{jh} \dot{\mathbf{e}}\right]$ of a Wide-Angle Conical Antenna . . . 47-56

Transmission Transfer Function of Conical Antenna With Matched Source.

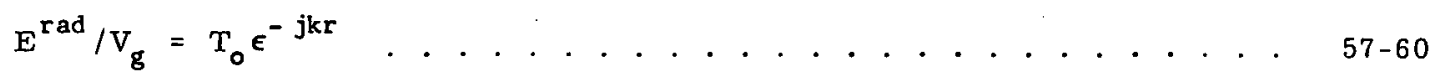

Reception Transfer Function per Unit of Antenna Length of Conical Antenna With Matched Load. $\mathrm{V}_{\mathrm{L}} / \mathrm{E}^{\mathrm{inc}}=\mathrm{S}$. . . . . . . . . . . . . . . . . . 61-63 
Figure 1 shows the conical antenna and its zero-resistivity ground plane with a coaxial line of characteristic impedance $Z_{k}$ ohm connected to its terminals. The cone half-angle is designated $\theta_{0}$ and the radius of the sphere that truncates the cone (hereinafter called the antenua length) is designated a meters. The input impedance of the antenna at its terminals is designated $Z_{0}(f) \mathrm{ohm}$.

In transmission, the coaxial line and its driving generator, whose impedance is matched to the line, can be represented by an equivalent source of open-circuit voltage $v_{g}(t)$ vo.t. and internal impedance $Z_{k}$ ohm connected directly to the antenna terminals. (See Figure 2.) We consider only the case where the equivalent source (i. e., the line) is matched to the antenna in the sense that

$$
\begin{aligned}
Z_{k} & =z_{c} \\
& \equiv \lim _{a f \longrightarrow \infty} Z_{0}(f) .
\end{aligned}
$$

(The limiting value $Z_{c}$ of antenna impedance is called the "characteristic" impedance* of the antenna, For conical antennas, $Z_{c}$ is a real and positive constant which depends on $\theta_{0}$.) We will show the time history $e^{r a d}(t)$ volt/meter of the radiation field in the equatorial plane (where $\theta=\pi / 2--$ see Figure 1 ) at the distance $\mathbf{r}=1000$ meters from the antenna for several values of length a and of cone half-angle $\theta_{0}$ when the equivalent-source voltage $v_{g}(t)$ volt is a pulse.

In reception, the line and its terminating impedance can be replaced at the antenna terminals by an equivalent load $Z_{L}(f)$ ohm. (See Figure 2.) We will show the time history of the voltage $v_{L}(t)$ volt across the load $Z_{L}(f)$ at the antenna terminals when the antenna is excited by a vertically-polarized plane wave $e^{i n c}(t)$ volt/meter of pulse form when, as in transmission, $Z_{L}(f)=Z_{c}$ (i.e., $Z_{k}=Z_{c}$ and impedance load on line is equal to $Z_{k}$ ), and, in addition, for a special case when $Z_{L}(f) \neq Z_{c}$.

Two excitation-pulse shapes are used for $v_{g}(t)$ volt in transmission; only one is used for $e^{\text {inc }}(t)$ volt/meter in reception. That used for both is a Gaussian pulse of unit amplitude and of width $t_{w}$ sec at $1 / 2$ unit. The other, used only in transmission, is designated "almost rectangular of 1-volt amplitude" since it results from truncating the spectrum of a 1 -volt rectangular pulse of width $t_{w}$ at the frequency $20 / \pi \mathrm{t}_{\mathrm{w}} \mathrm{Hz} .^{\dagger}$ Both are plotted in Figure 3 against the variable $t / t_{w}$ which is time divided by the pulse width.

* It is similar to the characteristic impedance of a transmission line in that it is the impedance as the length a increases without limit. See Sec. 3.4a.

${ }^{\dagger}$ We use hertz, abbreviated $\mathrm{Hz}$, as the unit of cyclic frequency $\mathrm{f}$; it has dimension $\mathrm{sec}^{-1}$. Thus, 1 hertz $\equiv 1$ cycle per second.

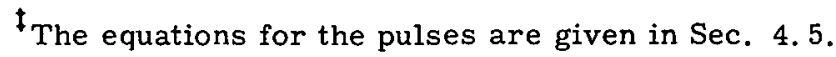




\section{2: Précis}

We here give a complıte preview of numerical results before proceeding to the analysis.

\subsection{Transmission}

The pulse $v_{g}(t)$ volt of the equivalent source connected to the antenna terminals is centered at $t=0$. Figures 4 through 8 show the resultant radiation field $e^{\text {rad }}(t)$ volt/meter in the equatorial plane where $\theta=\pi / 2$ at 1000 meters from the antenna (translated $\mathrm{r} / \mathrm{c}=10^{-5} / 3 \mathrm{sec}$, where $\mathrm{c}=3 \times 10^{8} \mathrm{~m} / \mathrm{sec}$, to remove the delay due to the 1000 meters of travel). Several values of cone half-angle $\theta_{0}$ are considered as well as both excitation-pulse shapes. The parameter $a / t_{w}$ is the antenna length divided by the pulse width and is given in meters per nanosecond. We note that, as $\theta_{0}$ increases, a/t $t_{w}$ must also increase to provide good reproduction of the excitation pulse.

\subsection{Reception}

The center of the pulse $e^{\text {inc }}(t)$ volt/metcr of the vertically-polarized incident field arrives at the antenna axis at $t=0$. Figures 9,10 , and 11 give the resultant voltage $v_{I_{1}}(t)$ voll acrusis lie eyuivaleilload impedance $Z_{L}(f)$ at the antenna terminals for the matched case where $Z_{L}$ (f) $=Z_{c}$. (The figures actually show $v_{L}(t)$ divided by excitation-pulse width $t_{w}$ sec.) Only Gaussian-pulse excitation is considered. We note that the incident-pulse shape is not reproduced across the load in any of the cases shown.

If we have a resistive load $R_{L}$ at the antenna terminals, and if all significant frequencies $f$ present in $e^{\text {inc }}(t)$ volt/meter satisfy the two inequalities

$$
1 / 2 \pi \mathrm{R}_{\mathrm{L}} \mathrm{C}_{\mathrm{a}} \ll \mathrm{f} \ll \mathrm{c} / 2 \pi \mathrm{a} \mathrm{Hz}
$$

where $C_{a}$ farad is the antenna capacitance* given by Equation (24) herein, then the voltage $v_{L}(t)$ across $R_{L}$ will be a faithful reproduction of the incident pulse and is given by

$$
v_{L}(t)=-h_{e}(0) e^{i n c}(t) \text { volt, }
$$

where $h_{e}(0)$ is the limiting value of the effective height $h_{e}(f)$ meter of the antenna as frequency $f$ approaches zero, and is given by Equation (27). The two inequalities mean that, at all significant frequencies in $e^{i n c}(t)$, the load resistance $R_{L}$ at the antenna terminals must be quite large compared to the antenna impedance $Z_{0}=1 / j 2 \pi \mathrm{fC}_{a}$ and that the antenna length a meter must be quite small compared to the wavelengths of those frequencies.

If the load on the antenna is a capacitance $C_{L}$, and if all significant frequencies in $e^{\text {inc }}(t)$ satisfy the single inequality

$$
f \ll c / 2 \pi a,
$$

\footnotetext{
*Under the condition $\mathrm{ka} \ll 1$, where $k \equiv 2 \pi / \lambda$, the antenna impedance is $Z_{0}=1 / j 2 \pi f C_{a}$.
} See Sec. 3. 3 . 
which is to say that $a$ is small compared to the wavelengths of all significait frequencies, then antenna and load together form a capacitive voltage divider so that $v_{L}(t)$ is a faithful reproduction of the incidentpulse shape and is given by

$$
v_{L}(t)=-\frac{C_{a}}{C_{a}+C_{L}} h_{e}(0) e^{i n c}(t) \text { volt. }
$$

If the antenna is long, i. e., if $\mathrm{ka} \rightarrow \infty$, if the load is

$$
Z_{L}(f)=R_{1}+1 / j 2 \pi f C_{1}
$$

and if

$$
2 \pi f\left(R_{1}+z_{c}\right) C_{1} \ll 1
$$

for all frequencies $f$ where $e^{i n c}(t)$ has significant components, then the voltage across $R_{1}$ is a faithful reproduction of the incident pulse and is given by

$$
v_{L}(t)=c R_{1} C_{1} e^{i n c}(t) \text { volt. }
$$

\section{Analysis}

Computation of the response of transmitting and receiving antennas to pulse excitation can readily be done through use of Fourier transforms provided both the steady-state driving-point, or input, impedance $Z_{0}(f)$ and the steady-state effective length $h_{e}(f)$ are knownitor the range of frequencies over which the excitation has significant amplitudes. Thus, the computation of the far-zone field of an antenna driven by a pulsed voltage source or the computation of the voltage across the terminals of an impedanceloaded antenna driven by a pulsed electromagnetic field requires knowledge of both $Z_{o}(f)$ and $h_{e}(f)$ over the frequency range of the pulse. Of course, the open-circuit voltage of a receiving antenna can be determined from $h_{e}(f)$ alone by its very definition. Also, the reflected voltage on a transmission line with an antenna load can be completely determined from $Z_{0}(f)$ and the characteristic impedance $Z_{k}$ of the line.

Happily, the theory of some classes of wave launchers and collectors, ${ }^{1-6}$ notably cylindrical and wide-angle conical antennas, has been developed sufficiently to permit calculation of the transfer functions relating source voltage to radiated field and incident field to load voltage over the range of frequencies required in the present study of transients. Presumably, for other antenna types, experiments can be devised which will permit the measurement of $Z_{0}(f)$ and $h_{e}(f)$ over an adequate range of frequencies.

The first step of this study consists of extending the range of frequencies over which numerical values of $Z_{0}(f)$ and $h_{e}(f)$ are currently available in published form for the wide-angle conical antenna.

\section{1 Driving-Point Impedance}

The driving-point impedance $Z_{0}(f)$ of the antenna pictured in Figure 1 is the ratio of the frequencydomain representation $V_{0}(f) v / H z$ of the voltage applied at the antenna terminals to the frequency-domain 
representation $I_{0}(f) a m p / F[z$ of the resultant current flowing into the antenna terminals. * The applied voltage $V_{o}(f)$ is approximately the line integral of the $\theta$-component $E_{\theta}(f)$ of the electric-field vector from the cone to the plane along an arc $r=\rho$, where $\rho$ is the inner radius of the outer conductor of the coaxial line, provided $\mathrm{k} \rho \ll 1$. The total current on the cone crossing the circle $\theta=\theta_{0}, \mathbf{r}=\rho$, which is approximately equal to $I_{o}(f)$ if $k \rho \ll 1$, is obtained by integrating the $\phi$-component $H_{\phi}(f)$ of the magnetic-field vector around the vertex of the cone. Thus,

$$
V_{0}(f) \doteq \int_{\theta_{0}}^{\pi / 2} E_{\theta}(f) \rho d \theta \text { volt } / H z
$$

and

$$
I_{0}(f) \doteq \int_{0}^{2 \pi} H_{\phi}(f) \rho \sin \theta_{0} d \phi a m p / H z
$$

Papas and King ${ }^{2}$ have derived expressions for $E_{\theta}(f)$ and $H_{\phi}(f)$ in the antenna region $\rho \leq r \leq a$, $\theta_{0} \leq \theta \leq \pi / 2$. They are

$$
\mathrm{H}_{\phi}(f)=\frac{\alpha E^{-j k r}+\rho e^{j k r}}{r \sin \theta}(\mathrm{amp} / \mathrm{m}) / \mathrm{Hz}
$$

and

$$
E_{\theta}(f)=\zeta_{0}\left(\frac{\alpha \epsilon^{-j k r}-\beta \epsilon^{j k r}}{r \sin \theta}\right)(\operatorname{volt} / m) / \mathrm{Hz},
$$

where $\alpha$ and $\beta$ are complex functions of frequency $f$, and $\zeta_{0}=120 \pi$ ohms is the characteristic resistance of space. If we assume that $k \rho \ll 1$, it follows that

$$
\begin{aligned}
V_{0}(f) & =\zeta_{0} \alpha(1-\beta / \alpha) \int_{\theta_{0}}^{1 / 2} \frac{\mathrm{d} \theta}{\sin \theta} \\
& =2 \pi Z_{c} \alpha(1-\beta / \alpha),
\end{aligned}
$$

and

$$
I_{0}(f)=2 \pi \alpha(1+\beta / \alpha)
$$

where

$$
z_{c} \equiv\left(\zeta_{0} / 2 \pi\right) \ln \cot \left(\theta_{0} / 2\right) \text { ohm. }
$$

*Werever we indicate a dependence on $f$ for a physical quantity which evidently has time variation, we are using its frequency-domain representation. See Secs 4. 1 and 4.2. 
The driving-point impedance is, therefore,

$$
\begin{aligned}
Z_{o}(f) \equiv V_{o}(f) / I_{o}(f) \\
=Z_{c} \frac{1-\beta / \alpha}{1+\beta / \alpha} \text { ohm. }
\end{aligned}
$$

The ratio $\beta / \alpha$ appearing in Equations $(5),(6)$, and $(8)$ is frequency-dependent and is given by ${ }^{2}$

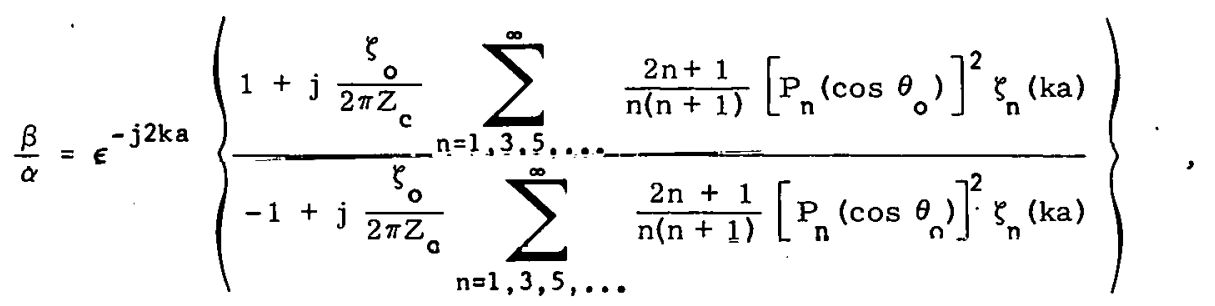

provided $30^{\circ} \leq \theta_{0}<90^{\circ}$, where

$$
\zeta_{n}(k a)=\frac{h_{n}^{(2)}(k a)}{h_{n-1}^{(2)}(k a)-\frac{n}{k a} h_{n}^{(2)}(k a)}
$$

$P_{n}(\cos \theta)$ is the Legendre polynomial of order $n$, and $h_{n}^{(2)}(x)$ is the spherical Hankel function of the second kind.

\section{2 Effective Length}

In an additional paper, Papas and King ${ }^{2}$ have derived an expression for the far-zone electric field of a wide-angle conical antenna; only the $\theta$-component is nonzero, of course. At the locus of points $(r, \theta)$ it is

$$
E^{r a d}(f ; r, \theta)=K \frac{\epsilon^{-j k r}}{k r} \sum_{n=1,3,5, \ldots}^{\infty} P_{n}\left(\cos \theta_{o}\right) P_{n}^{1}(\cos \theta)\left(\frac{2 n+1}{n^{2}+n}\right)\left\{\frac{\cdots}{h_{n-1}^{(2)}(k a)-\frac{n}{k a} h_{n}^{(2)}(k a)}\right\}
$$

where

$$
K=\frac{\zeta_{0} \alpha}{a}\left(\epsilon^{-j k a}-\frac{\beta}{\alpha} \epsilon^{j k a}\right),
$$

where $P_{n}^{l}(z)$ is the modified Legendre polynomial of order $n$ and which satisfies,

$$
P_{n}^{1}(\cos \theta)=-\frac{d}{d \theta} P_{n}(\cos \theta)
$$

and where $r$ is the distance from the point of field calculation in the radiation zone to the vertex of the cone. From this point on, we will be concerned only with the radiation field in the equatorial plane where $\theta=\pi / 2$; we designate it $\mathrm{E}^{\text {rad }}(f)$. 
The radiation-field pattern of a two-terminal antenna whose radiation field is geometrically dependent only on $\theta$ and $r$ (and not on $\phi$--see Figure 1 ) is defined for the frequency $f$ by

$$
F(f ; \theta) \equiv \frac{E^{r a d}(f ; r, \theta)}{\left(j \zeta_{0} / 2 \pi\right)\left(\epsilon^{-j k r} / r\right) I_{0}(f)}
$$

Further, it is a consequence of the Rayleigh-Carson reciprocity theorem that, for any two-terminal antenna, ${ }^{7}$

$$
\mathrm{kh}_{\mathrm{e}}(f ; \theta)=F(f ; \theta)
$$

where $h_{e}(f ; \theta)$ meter is the complex effective lenglh of the antenna at the frequency $f$. We wish to find the effective length for vertically-polarized incident radiation, 1 . e., for $0 \equiv . \pi / 2$, and we deislynule il $h_{e}(f)$.

We use (6) to find the constant $\alpha$ in terms of the input current $I_{0}(f)$ and the ratio $\beta / \alpha$ :

$$
\alpha=\frac{I_{0}(f)}{2 \pi(1+\beta / \alpha)}
$$

We now substitute (16) into (12) and the result into (11) in which we also take $\theta=\pi / 2$. This leads to an expression for the radiation field at distance $r$ from the antenna for $\theta=\pi / 2$. which we write as $E^{r a d}(f)$ :

$$
\begin{aligned}
& E^{r a d}(f)= \frac{\zeta_{0} I_{0}(f)}{2 \pi k a\left(1+\frac{\beta}{\alpha}\right)}\left(\epsilon^{-j k a}-\frac{\beta}{\alpha} \epsilon^{j k a}\right) \frac{\epsilon^{-j k r}}{r} \\
& \sum_{n=1,3,5, \ldots}^{\infty} P_{n}\left(\cos \theta_{0}\right) P_{n}^{1}(0)\left(\frac{2 n+1}{n^{2}+n}\right) \cdot \frac{j^{n}}{h_{n-1}^{(2)}(k a)-\frac{n}{k a} h_{n}^{(2)}(k a)}
\end{aligned}
$$

The ratio $\beta / \alpha$ is given by (9). Putting (17) into (14) allu using (13), with $\theta=n / 3$, gives

$$
\begin{aligned}
k_{e}= & \frac{1}{k a\left(1+\frac{\beta}{\alpha}\right)}\left(\frac{\beta}{\alpha} \epsilon^{j k a}-\epsilon^{-j k a}\right) \\
& \sum_{n=1,3,5}^{\infty} P_{n}\left(\cos \theta_{0}\right) P_{n}^{1}(0)\left(\frac{2 n+1}{n^{2}+n}\right) \frac{j^{n+1}}{h_{n-1}^{(2)}(k a)-\frac{n}{k a} h_{n}^{(2)}(k a)} .
\end{aligned}
$$

This is the final expression for the radian effective length of the wide-angle conical antenna pictured in Figure 1 for the reception of vertically-polarized plane waves, i. e., for the case where the incident electric-field vector is directed vertically. 


\section{3 Impedance and Effective Length of a Short, Wide-Angle Conical Antenna}

We now develop formulas for the impedance and effective length of a wide-angle conical antenna for which ka $\ll 1$.

3. 3a Impedance--Since

$$
h_{n}^{(2)}(x)=\sqrt{\frac{\pi}{2 x}} H_{n+1 / 2}^{(2)}(x),
$$

and

$$
H_{n}^{(2)}(x)=J_{n}(x)-j Y_{n}(x) \text {, }
$$

$\zeta_{1}(\mathrm{ka})$, as defined by $(10)$, becomes

$$
\zeta_{1}(k a)=\frac{h_{1}^{(2)}(k a)}{h_{0}^{(2)}(k a)-\frac{1}{k a} h_{1}^{(2)}(k a)} \doteq-\frac{k a}{1+j k a} .
$$

Noting that $\left[P_{1}\left(\cos ^{2} \theta_{0}\right)\right]^{2}=\cos ^{2} \theta_{0}$ in (9), (8) becomes

$$
z_{0} \doteq-j \frac{4 \pi z_{c}^{2}}{k a\left(4 \pi z_{c}+3 \zeta_{o} \cos ^{2} \theta_{o}\right)} .
$$

This is the final formula for the impedance of an electrically-short, wide-angle conical antenna. It is a pure reactance. The impedance of an electrically-short, cylindrical monopole is ${ }^{8}$

$$
z_{0}=-j \frac{\zeta_{0}}{4 \pi \mathrm{kh}}[\Omega-2-2 \& \mathrm{n} 2]
$$

where $\Omega=2 \ln \left(2 \mathrm{~h} / \mathrm{a}_{0}\right), \mathrm{h}$ is the height of the monopole, and $\mathrm{a}_{0}$ is its radius. It is to be observed that (22) and (23) are comparable in all respects, i. e., in both cases the impedance is that of a capacitor whose capacitance is proportional to length.

It is clear by Equation (22), that, when ka $\ll 1$, the wide-angle conical antenna is equivalent to a lumped capacitor of value

$$
c_{a}=\frac{a\left(4 \pi z_{c}+3 \zeta_{o} \cos ^{2} \theta_{o}\right)}{4 \pi c z_{c}^{2}} \text { farad. }
$$

If $\theta_{0}=46.982^{\circ}$, then $Z_{c}=50 \mathrm{ohms}$. Let $\mathrm{ka}=0.1$. The impedance, as given by (22), is $z_{0}=0-j 272.2 \mathrm{ohms}$. The impedance given in the appropriate table for this case is $z_{0}=0.12124-j 263.96$ ohms. (The latter result is based on taking 29 terms in the series; i. e., the series was truncated following $n=57$.) The error in the real part of $z_{0}$ given by (22) relative to the absolute value $\left|z_{0}\right|$ is 0.05 percent, and the relative error in the imaginary part is 3 percent. 
3. 3b Effective Length--The leading term in (18) for $\mathrm{ka} \ll 1$ is

$$
\mathrm{kh}_{e}(\mathrm{f})=-\frac{\mathrm{j} 3}{2 \mathrm{ka}}\left(\frac{1-\frac{\beta}{\alpha}}{1+\frac{\beta}{\alpha}}\right) \cos \theta_{0} \mathrm{P}_{1}^{1}(0)\left[\frac{1}{\mathrm{~h}_{0}^{(2)}(\mathrm{ka})-\frac{1}{\mathrm{ka}} \mathrm{h}_{1}^{(2)}(\mathrm{ka})}\right]
$$

The factor $(1-\beta / \alpha) /(1+\beta / \alpha)$ is available from $(8)$ and $(22)$; reference to (13) shows that $P_{1}^{1}(0)=1$ when $\theta=\pi / 2$. Also,

$$
h_{0}^{(2)}(k a)-\frac{1}{k a} h_{1}^{(2)}(k a) \doteq \frac{1}{(k a)^{2}}+j\left[\frac{1}{k a}-\frac{1}{(k a)^{3}}\right] \text {, }
$$

provided ka $<<1$. With the above, (25) becomes

$$
\mathrm{kh}_{e}(f)=\frac{6 \pi \mathrm{kaz} c \cos \theta_{0}}{4 \pi z_{c}+3 \xi_{0} \cos ^{2} \dot{\theta}_{0}} .
$$

This result is formally similar to that obtained for the short cylindrical monopole. F'or this case, King ${ }^{9}$ obtains

$$
\mathrm{kh}_{\mathrm{eo}}(\mathrm{f})=\frac{\mathrm{kh}(\Omega-1)}{2(\Omega-2+\ell \mathrm{n} 4)} \text {. }
$$

where $h_{e o}(f)$ is the effective length of the vertical monopole for a vertically-polarized incident field. Note that for $\mathrm{ka} \ll 1, \mathrm{kh}_{\mathrm{e}}$ and $\mathrm{kh}_{\mathrm{eo}}$ are real.

As before, if $\theta_{0}=46.982^{\circ}$, then $z_{c}=50$ ohms. If $\mathrm{ka}=0.1$, (27) gives $\mathrm{kh}_{e}=0.0557$. The machine-computed value for this case, based on 29 terms (to $11=57$ ) in the series, ic

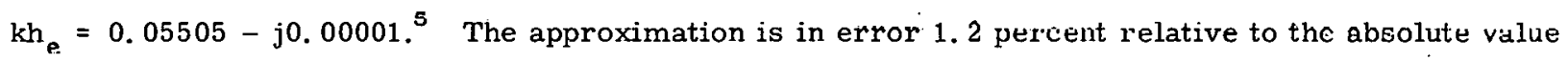
$\left|\mathrm{kh}_{\mathrm{e}}\right|$ for the real part of $\mathrm{kh}_{\mathrm{e}}$, and is in relative error of 0.02 percent for the imagtilary part.

\section{4 Impedance and Effective Length of a Long, Wide-Angle Conical Antenna}

We now consider formulas for the impedance and effective length of a wide-angle contcal anlentid for which $\mathrm{ka}$ increases without limit.

3. 4a Impedance--Since the ratio $\beta / \alpha$ given by Equation (9) approaches zero* as ka increases without limit, we have by Equation (8)

$$
z_{c}=\lim _{k a \rightarrow \infty} Z_{o}(f)
$$

Thus, $Z_{c}$ is the limiting value of $Z_{o}(f)$ as either frequency $f=c k / 2 \pi$ or antenna length a approaches infinity. Since the characteristic impedance of a transmission line is the input impedance as its length increases. without limit, we define $Z_{c}$ as the characteristic impedance of the conical antenna. (In circuit theory, characteristic impedance is usually defined only for symmetrical two-port networks. ) It is clear from Equation (7) that $Z_{c}$ is positive and real since $0 \leq \theta_{0} \leq \pi / 2$ and is not dependent on frequency $f$ or length a.

\footnotetext{
* See Sec. 4. 6.
} 
3. $4 \mathrm{~b}$ Effective Length--It can be shown* that

$$
\lim _{k a \rightarrow \infty} \operatorname{kh}_{e}(f)=-j
$$

which is to say that $h_{e}(f) \rightarrow-c / j 2 \pi f$ as either frequency $f$ or length a increases without limit. Since $h_{e}(f)$ is a transfer function that relates the antenna open-circuit voltage to the incident field, the time history of the open-circuit voltage is proportional to the integral of the time history of the incident field for the case of a long antenna. ${ }^{\dagger}$

\section{5 Numerical Results for Impedance and Effective Length}

Figures 12 through 21 provide $Z_{0}(f)=R_{0}(f)+j X_{0}(f)$ over the range $0 \leq k a \leq 20$, with $\theta_{0}$ as a parameter, for the antenna shown in Figure 1. Figures 22 through 31 show the real and imaginary parts of $\mathrm{kh}_{\mathrm{e}}(\mathrm{f})$, the radian length, over the same range. It is clear that impedance matching of this antenna over a wide frequency band is simple. However, since the transfer functions of the antenna for both transmission and reception depend on $h_{e}(f)$ as well as on $z_{0}(f)$, it cannot in general faithfully transmit or receive wide-band signals.

It should be pointed out that 29 terms in the several series were taken in evaluating $Z_{0}(f)$ and $\mathrm{kh}_{\mathrm{e}}(\mathrm{f})$; that is, truncation followed $\mathrm{n}=57$. It is assumed that the numerica: results so obtained, even for large $\mathrm{ka}$, are accurate.

\subsection{Transfer Function of Antenna and Source in Transmission}

From Figure 2, the relation between source voltage $V_{g}(f)$. and antenna current $I_{0}(f)$ is

$$
I_{0}(f)=\frac{V_{g}(f)}{Z_{0}(f)+Z_{k}} \operatorname{amp} / \mathrm{Hz}
$$

This relation, together with (17) and (18), gives

$$
E^{r a d}(f)=j \frac{\zeta_{0}}{2 \pi} \frac{V_{g}(f)}{Z_{o}(f)+Z_{k}} \frac{\epsilon^{-j k r}}{r} k h_{e}(f)(v / m) / H z
$$

The transmission transfer function is, then,

$$
\begin{aligned}
T(f) & \equiv \frac{E^{r a d}(f)}{V_{g}(f)} \text { (volt/meter)/volt } \\
& =\frac{j \zeta_{0} f}{c r} \frac{h_{e}(f)}{Z_{o}(f)+Z_{k}} \epsilon^{-j(2 \pi r / c) E} \\
& =T_{0}(f) \epsilon^{-j(2 \pi r / c) E}(v / m) / v
\end{aligned}
$$

\footnotetext{
* See Sec. 4.7.

$t_{\text {See Sec. 4. } 3 .}$

$\$$ See Sec. 4. 2 .
} 
where the definition of $T_{0}(f)$ is evident, and where we have made the substitution $k \equiv 2 \pi f / c$. The exponential factor $\epsilon^{-j(2 \pi r / c) f}$ has the sole effect in the time domain of providing a time lag of $\mathrm{r} / \mathrm{c}$ seconds. This, of course, is just the time required for the wave to travel the distance $r$ meters.

Figures 32, 33, and 34 show $T_{0}(f)$ as a function of frequency $f$ for $r=1000$ meters, for $Z_{k}=Z_{c}$, and for three values of cone half-angle $\theta_{0} . T_{0}(f)$ depends on $f$ and on the antenna parameters $a$ and $\theta_{0}$. Because of the condition $Z_{k}=Z_{c}$, the dependence on both a and $f$ is solely through the product af, or $k a=2 \pi$ af $/ c$.

\subsection{Time History of the Radiated Field}

We have taken $E^{r a d}(f)$ to be the frequency-domain representation of the time function $e^{\text {rad }}(t)$. The latter is given in terms of the former by

$$
\begin{aligned}
e^{r a d}(t) & =\int_{-\infty}^{\infty} E^{r a d}(f) \epsilon^{j 2 \pi f t} d f \\
& =\int_{-\infty}^{m} V_{g}(f) T(f) \epsilon^{j 2 \pi f t} d f \\
& =\int_{-\infty}^{\infty} V_{g}(f) T_{0}(f) \epsilon^{j 2 \pi(t-r / c) f_{d f} \text { volt } / \text { meter }}
\end{aligned}
$$

which is readily transformed to

$$
e^{r a d}(t+r / c)=\int_{-\infty}^{\infty} V_{g}(f) T_{n}(f) \epsilon^{j 2 \pi f t} d f v / m
$$

$V_{g}(f)$ is the frequency-domain representation of $v_{g}(t)$ and is, therefore,

$$
V_{E}(f)=\int_{-\infty}^{\infty} v_{g}(t) \epsilon^{-j 2 \pi f t} d t v / H z
$$

Hence, if the exciting waveform $v_{g}(t)$ and the transfer function $T_{0}(f)$ are known, the response $e^{r a d}(t+r / c)$ can be determined. We have used numerical integration to find it for the various cases shown in Figures $4,5,6,7$, and 8 . Because of the condition $Z_{k}=Z_{c}$, we are able to reduce the three parameters $a, t_{w}$, and $\theta_{0}$ to the two $a / t_{w}$ and $\theta_{0}$ by plotting $e^{r a d}(t+r / c)$ against $t / t_{w}$ :

\subsection{Transfer Function of Antenna and Load in Reception}

From Figure 2, the relation between load voltage $V_{L}(f)$ and incident field $E^{\text {inc }}(f)$ is

$$
V_{L}(f)=\frac{-h_{e}(f) E^{i n c}(f) Z_{L}(f)}{Z_{0}(f)+Z_{L}(f)} \text { volt } / H z
$$


Hence, the reception transfer function is.

$$
\begin{aligned}
S(f) & =\frac{V_{L}(f)}{E^{\text {inc }}(f)} \\
& =\frac{-h_{e}(f) Z_{L}(f)}{Z_{0}(f)+Z_{L}(f)} \text { (volt)/(volt/meter). }
\end{aligned}
$$

Figures 35,36 , and 37 give $S(f)$ as a function of frequency $f$, for $Z_{L}(f)=Z_{c}$, and for three values of cone half-angle $\theta_{0} . S(f)$ depends on $f$ and on the antenna parameters a and $\theta_{0}$. Because of the condition $Z_{L}(f)=Z_{c}$, the dependence of the ratio $S(f) / a$ on both a and $f$ is solely through the product af, or $\mathrm{ka}=2 \pi \mathrm{af} / \mathrm{c}$.

Also from Figure 2, the short-circuit load current at the terminals of the conical antenna is

$$
I_{\text {Lsc }}(f)=\frac{-h_{e}(f) E^{i n c}(f)}{Z_{0}(f)} \mathrm{amp} / \mathrm{Hz} .
$$

The time history of the short-circuit current can readily be obtained from this relation.

\section{9 Time History of Load Voltage} then,

$V_{L}(f)$ is the frequency-domain representation of the time function $v_{L}(t)$. of load voltage. We have,

$$
\begin{aligned}
v_{L}(t) & =\int_{-\infty}^{\infty} V_{L}(f) \epsilon^{j 2 \pi f t} d f \\
& =\int_{-\infty}^{\infty} E^{i n c}(f) S(f) \epsilon^{j 2 \pi f t} d f \text { volt. }
\end{aligned}
$$

$E^{\text {inc }}$ (f) is determined from $e^{\text {inc }}(t)$ by

$$
E^{i n c}(f)=\int_{-\infty}^{\infty} e^{i n c}(t) \epsilon^{-j 2 \pi f t} d t .
$$

The integral above for $v_{L}(t)$ was integrated numerically to find $v_{L}(t)$ as shown in Figures 9 , 10, and 11. Because of the condition $Z_{l}(f)=Z_{c}$, we are able to reduce the three parameters a, $t_{w}$, and $\theta_{0}$ to the two $a / t_{w}$ and $\theta_{0}$ by plotting $v_{L}(t) / t_{w}$ against $t / t_{w}$.

\subsection{Transmission Transfer Function for Short Antenna With Mat'sed Source}

We take ka $<1$ and $Z_{k}=Z_{c}$. Then, in $T_{0}(f)$ as defined by Equation (33) we substitute $Z_{0}$ (f) as given by Equation (22) and $h_{e}(f)$ as given by Equation (27) and set $Z_{k}=Z_{c}$ to obtain

$$
T_{0}(f)=(j 2 \pi f)^{2} \frac{3 \xi_{o} a^{2} \cos \theta_{0}}{4 \pi Z_{c} r c^{2}}(v / m) / v .
$$


Since multiplication in the frequency domain by the term $j 2 \pi f$ corresponds to time differentiation in the time domain, ${ }^{*}$ we have, besause of the factor $(j 2 \pi f)^{2}$ in Equation (41), that the short transmitting antenna (i. e., where ka $<<1$ ) with the matched source (i. e., where $z_{k}=z_{c}$ ) produces a waveform of radiated field that is, in shape, the second derivative of the source voltage. That is, from Equation (41)

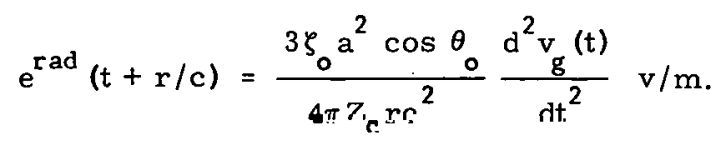

A tendency toward double differentiation can be observed in Figure 5 for the case where $a / t_{w}=0.0883$ $\mathrm{m} / \mathrm{nsec}$. This corresponds approximately to $\mathrm{ka}=1$ for the maximum significant frequency in the pulse; hence, double differentiation can only be expected to be approximate in this case.

\subsection{Transmission Transfer Function for Long Antenna With Matched Source}

Let $\mathrm{ka}$ increase without limit and let $Z_{k}=Z_{c}$. Then, in $T_{0}(f)$ as defined by Equation (33) we substitute $Z_{o}(f)$ as given by Equation (29), $h_{e}(f)$ as given by Equation (30), and set $Z_{k}=Z_{c}$ to obtain

$$
T_{0}(\rho)=\frac{\zeta_{0}}{4 \pi Z_{c}}(v / 11) / v
$$

For this case the transfer function is a constant; hence, the pulse of radiated field is of the same shape as the source pulse. That is,

$$
e^{r a d}(t+r / c)=\frac{\zeta_{0}}{4 \pi r z_{c}} v_{g}(t) v / m
$$

We observe this reproduction of the source pulse in Figure 5 for the case where $a / t_{w}=6.62 \mathrm{~m} / \mathrm{nsec}$ (which corresponds to $\mathrm{ka} \doteq 10$ for the minimum significant frequency in the pulse). $\overline{\mathrm{F}}_{\mathrm{or}} \xi_{\circ}=120 \pi$ ohms, $r=1000$ meters, and $Z_{c}=50 \mathrm{ohms}$, we find $\zeta_{o} / 4 \pi \mathrm{rZ} Z_{c}=0.6 \times 10^{-3} \mathrm{~m}^{-1}$. Since the peak value of $v_{g}(t)$ is 1 volt, the peak of $e^{r a d}(t+r / c)$ is $0.6 \times 10^{-3} . v / m$; we observe this value in Figure 5 .

\subsection{Reception Transfer Function for Short Antenna With Matched Load}

I et. ka $\ll 1$ and $Z_{T}(f)=Z_{r}$. Then, in $S(f)$ as defined by Equation (38), we substitute $Z_{0}$ (f) as given by Equation (22) and $h_{e}(f)$ as given by Equation (27), and set $Z_{L}(f)=Z_{c}$ to obtain

$$
S(f)=-j 2 \pi f \frac{3 a^{2} \cos \theta_{0}}{2 c} v /(v / m) \text {. }
$$

The term $j 2 \pi f$ corresponds to differentiation in the time domain. Thus,

$$
v_{L}(t)=-\frac{3 a^{2} \cos \theta_{0}}{2 c} \frac{d e^{i n c}(t)}{d t} \text { volt. }
$$

In Figure 10 for the case $a / t_{w}=0.0883 \mathrm{~m} / \mathrm{nsec}$, we observe a pulse shape that approximates the derivative of the incident pulse.

\footnotetext{
${ }^{*}$ See Sec. 4.3
} 


\subsection{Reception Transfer Function for Long Antenna With Matched Load}

Let $k a \rightarrow \infty$ and let $Z_{L}(f)=Z_{c}$. Then, in $S(f)$ as given by Equation (38), we substitute $Z_{0}(f)$ as given by Equation (29), $h_{e}(f)$ as given by Equation (30), and set $z_{L}(f)=Z_{c}$ to obtain

$$
S(f)=-\frac{1}{j 2 \pi f} \cdot \frac{c}{2} v /(v / m) \text {. }
$$

The term $1 / j 2 \pi f$ corresponds to integration in the time domain. ${ }^{*}$ Hence

$$
v_{L}(t)=-\frac{c}{2} \int_{-\infty}^{t} e^{i n c}(\tau) d \tau \text { volt. }
$$

Since, for Gaussian excitation, the area of the incident pulse multiplied by $c / 2$ is $0.16 t_{w}$ gigavolt, $-v_{L} / t_{w}$ should rise to and remain at 0.16 gigavolt $/ \mathrm{sec}$ or $160 \mathrm{mv} / \mathrm{nsec}$. To a first approximation this is true for the case $a / t_{w}=6.62 \mathrm{~m} / \mathrm{nsec}$ shown in Figure 10.

\subsection{Reception Characteristic of Long Antenna With Differentiating-Circuit Load}

Since the open-circuit voltage of a long antenna is proportional to the time integral of the time function of incident field, it is of interest to consider the effect of a differentiating-circuit load, i. e., the case where

$$
Z_{L}(f)=R_{1}+1 / j 2 \pi f C_{1}
$$

Since the impedance of the long conical antenna is. $Z_{c}$, the load current is, from Figure 2 and Equations (29) and (30).

$$
\begin{aligned}
I_{L}(f) & =\frac{(c / j 2 \pi f) E^{i n c}(f)}{R_{1}+Z_{c}+1 / j 2 \pi f C_{1}} \\
& =\frac{c C_{1} E^{i n c}(f)}{1+j 2 \pi f\left(R_{1}+Z_{c}\right) C_{1}} \\
& \doteq c C_{1} E^{i n c}(f) \operatorname{amp} / H z
\end{aligned}
$$

where the last step follows provided

$$
2 \pi f\left(R_{1}+z_{c}\right) C_{1} \ll 1
$$

for all frequencies $f$ where $E^{\text {inc }}(f)$ has significant components. We can say, then, that

$$
i_{L}(t)=c C_{1} e^{i n c}(t) a m p
$$

when the reactance of the capacitor $C_{1}$ is very much greater than the combined resistance $R_{1}+Z_{c}$ of load and antenna $\left(z_{c}\right.$ being real, of course) for all significant frequencies of the incident field $e^{i n c}(t)$ volt/meter.

\footnotetext{
* See Sec. 4. 3.
} 


\subsection{Reflected Voltage on the Line}

The time function of reflected voltage on a line driving an antenna (or other load) can be determined by the Fourier transform from the characteristic impedance of the line and the driving-point impedance of the antenna, provided these functions are known for the range of frequencies over which the spectrum of the excitation has significant components.

Let $e^{+}(t)$ be the forward-traveling voltage wave on the line as it appears at the load end of the line (i.e., across the antenna terminals). Let $e^{\prime}(t)$ be the reverse-traveling wave at the same place, and let $E^{+}(f)$ and $E^{-}(f)$ be their frequency-domain representations. These frequency-domain representations are related by

$$
E^{-}(f)=\left[\frac{Z_{0}(f)-Z_{k}}{Z_{0}(f)+Z_{k}}\right] E^{+}(f)
$$

where $Z_{0}(f)$ is the antenna impedance and $Z_{k}$ is the characteristic impedance of the line, since the expression in brackets is the voltage reflection coefficient at the load. The time function that describes the reflected wave is obtained by taking the inverse Fourier transform of $\mathrm{E}^{-}(\mathrm{f})$ :

$$
\begin{aligned}
e^{-}(t) & =\int_{-\infty}^{\infty} E^{-}(f) \epsilon^{j 2 \pi f t} d f \\
& =\int_{-\infty}^{\infty} \frac{Z_{0}(f)-Z_{k}}{Z_{0}(f)+Z_{k}} E^{+}(f) \epsilon^{j 2 \pi t} d f .
\end{aligned}
$$

Figures 12 through 21 show that when $k a=(2 \pi a / c) f \geq 4, Z_{0}(f)$ is approximately equal to $Z_{c}$. Hence, if the characteristic impedance $Z_{k}$ of the line is set equal to $Z_{c}$, and if the forward-traveling pulse $e^{+}(t)$ volts on the line does not have significant components at frequencies below $2 c / \pi a \mathrm{~Hz}$, then there will be no significant reflected pulse. (These remarks do not apply to a cylindrical antenna since the antenna impedance does not reach a constant asymptotic value such as $\left.Z_{c}.\right)^{10}$

\subsection{Remarks}

Tai Wu has suggested ${ }^{*}$ that pulse radiation occurs from the conical antenna as the pulse of charge passes from the transmission line onto the antenna, again as the charge turns the corner from the cone onto the spherical cap, and again when the charge turns the corner back to the cone after passing over the spherical cap. Thus, if the pulse center leaves the transmission line at $t=0$, the radiation pulse at distance $r$ from the antenna should have its center at $t=r / c$. This is verified in Figure 4 for the case where $a / t_{w}=2.21$. (The plots in the figures have been translated to the left $\mathrm{r} / \mathrm{c} \mathrm{sec}$ so that verification consists of finding the radiation-pulse center at $t=0$.)

Let $\Delta t_{1}$ be the time that the center of the radiation pulse due to the turn of the charge onto the cap lags the center of the initial radiation pulse. (We consider the radiation in the equatorial plane where

\footnotetext{
* Conversation with C. W. Harrison, Jr. in Cambridge, Massachusetts.
} 
$\theta=\pi / 2$ and suppose that the charge travels along the antenna at velocity c.) The charge reaches the turn at a/c sec; at the turn, it has moved in the horizontal direction a sin $\theta_{0}$ meters. Hence,

$$
\Delta t_{1}=\left(a-a \sin \theta_{0}\right) / c \sec
$$

or

$$
\Delta t_{1} / t_{w}=\left(a / t_{w}\right)\left(1-\sin \theta_{0}\right) / c
$$

If we take $a / t_{w}=2.21$ and $\theta_{0}=30^{\circ}$, we calculate

$$
\Delta t_{1} / t_{w}=3.69
$$

Tn Figire $4 \mathrm{fnr} a / t_{w}=2.2 .1$, we nhaperve that. $\wedge t_{1} / t_{w}=4, n$ :

Let $\Delta t_{2}$ be the lag of the radiation pulse due to the turn from the cap back to the cone behind the pulse due to the turn onto the cap. Between these two turns the charge travels the distance $2 \theta_{0}{ }^{a}$ meters. Hence,

$$
\Delta t_{2}=2 \theta_{0} a / c \sec
$$

or

$$
\Delta t_{2} / t_{w}=2 \theta_{0}\left(a / t_{w}\right) / c
$$

If we again take $a / t_{w}=2.21$ and $\theta_{0}=30^{\circ}$, we find

$$
\Delta t_{2} / t_{w}=7.71
$$

In Figure 4 we observe that $\Delta t_{2} / t_{w}=7.0$ for $a / t_{w}=2.21$.

The remarks above are pertinent to the length a of antenna needed to make the radiated pulse a faithful reproduction of the input-current pulse. Simply stated, the pulse due to the turn from cone to cap must not overlap the initial pulse, i. e.

$$
\Delta t_{1} \geq t_{b}
$$

where we define the base width $t_{b}$ sec as that width over which the pulse has "significant" amplitude. (In the case of the Gaussian pulse, we arbitrarily choose significant amplitude so that $t_{b}=2 t_{w}$; for $a$ rectangular pulse it is clear that $t_{b}=t_{w}$.) Substituting for $\Delta t_{1}$ in the inequality above and solving for a. gives

$$
a \geq c t_{b} /\left(1-\sin \theta_{0}\right) \text { meter }
$$

or

$$
a / t_{w} \geq c\left(t_{b} / t_{w}\right) /\left(1-\sin \theta_{0}\right) \cdot \operatorname{meter} / \text { sec. }
$$


We note that the length a must increase as the cone half-angle $\theta_{0}$ increases. For the Gaussian pulse the requirements for faithful transmission are

$$
\begin{aligned}
a / t_{w} & \geq 1.2 \mathrm{~m} / \mathrm{nsec} \text { for } \theta_{0}=30^{\circ}, \\
& \geq 2.2 \mathrm{~m} / \mathrm{nsec} \text { for } \theta_{0}=47^{\circ}, \\
& \geq 0.0 \mathrm{mir} / \mathrm{nsec} \text { fن } \iota^{\circ} 0_{0}-70^{\prime \prime} .
\end{aligned}
$$

(See Figures 4, 5, and 6 for verification of the numbers just computed.) For the rectangular pulse, they are

$$
\begin{aligned}
a / t_{w} & \geq 0.60 \mathrm{~m} / \mathrm{nsec} \text { for } \theta_{0}=30^{\circ}, \\
& >1.1 \mathrm{~m} / \mathrm{nsec} \text { for } \theta_{0} \cong 4^{\prime} !^{\circ} . \\
& \geq 4.9 \mathrm{~m} / \mathrm{nsec} \text { for } \theta_{\sigma}=70^{\circ} .
\end{aligned}
$$

(See Figures 7 and 8.)

When the charge moves along a vertical cylindrical monopole, it has no component of motion in the horizontal direction. The second pulse of radiation is due to the reversal of the direction of charge flow at the end. Hence,

$$
\Delta t_{1} / t_{w}=\left(h / t_{w}\right) / c
$$

where $h$ meter is the monopole length. Hence, the monopole length needed for faithful transmission is

$$
h / t_{w} \geq c\left(t_{b} / t_{w}\right) \text { meter/sec. }
$$

Thus, the length $\mathrm{h}$ of a monopole antenna is less than the length a of a conical antenna needed for faithrui transmission.

We remark that the computations of this section are of an approximate nature. Complete waveform calculations are needed to determine the degree of fidelity of transmission.

\section{Appendix}

\section{1 Frequency-Domain Representation of a Time Function}

We use this expression to mean the Fourier transform $V(f)$ of the time function $v(t)$, which as an example we take to represent a voltage, defined as follows:

$$
V(f)=\int_{-\infty}^{\infty} v(t) \epsilon^{-j 2 \pi f t} d t \operatorname{volt} / \mathrm{Hz} .
$$


We determine the time function from its frequency-domain representation by

$$
v(t)=\int_{-\infty}^{\infty} V(f) \epsilon^{j 2 \pi f t} d f \text { volt. }
$$

(We assume that all conditions required for the representation above to be proper do indeed exist.)

\section{2 Transfer Function of a Linear System}

If the input time function $i_{1}(t)$ of a linear system is

$$
i_{1}(t)=\operatorname{Re}\left\{\epsilon^{j 2 \pi f t}\right\}
$$

the resultant output time function $e_{1}(t)$ has the form

$$
e_{1}(t)=\operatorname{Re}\left\{z(f) \epsilon^{j 2 \pi f \tau}\right\}
$$

The complex function $Z(f)$ which depends only on the frequency $f$ is the transfer function of the system. (Since $\operatorname{Re}\left\{\epsilon^{j 2 \pi f t}\right\}=\operatorname{Re}\left\{\epsilon^{-j 2 \pi f t}\right\}$, it follows that $\operatorname{Re}\{Z(f)\}$ is an even funstion of $f$ while $\operatorname{Im}\{Z(f)\}$ is an odd function.) For example, if the system is an inductor of inductance $L$ henry with the current as the input and the voltage across it as the output, we have

$$
\begin{aligned}
e_{1}(t) & =L \frac{d i_{1}(t)}{d t} \\
& =\operatorname{Re}\left\{j 2 \pi f L \epsilon^{j 2 \pi f t}\right\}
\end{aligned}
$$

so that the transfer function is $Z(f)=j 2 \pi f L$ ohm which is, of course, the impedance of the inductor.

Let us suppose that we have a more general input time function of the form

$$
i(t)=\operatorname{Re} \int_{-\infty}^{\infty} I(f) \epsilon^{j 2 \pi f t} d f
$$

which is a sum of inputs of the form of $i_{1}(t)$ with amplitudes $I(f) d f$. By the definition of a linear system, the output due to $i(t)$ is

$$
e(t)=\operatorname{Re} \int_{-\infty}^{\infty} I(f) Z(f) \epsilon^{j 2 \pi f t} d f
$$

That is, the output due to an input $\operatorname{Re}\left\{I(f) \epsilon^{j 2 \pi f t} d f\right\}$ is $\operatorname{Re}\left\{I(f) Z(f) \epsilon^{j 2 \pi f t}\right.$ df $\}$, and the total output is the sum of such individual outputs.

If we place the condition that $\operatorname{Re}\{(f)\}$ be an even function while $\operatorname{Im}\{(f(f)\}$ be odd, it follows that we can drop the Re before the integral for $i(t)$ and write

$$
i(t)=\int_{-\infty}^{\infty} I(f) \epsilon^{j 2 \pi f t} d f .
$$


Thus, $i(t)$ can be any real time function that has a Fourier representation. Since the real and imaginary parts of $Z(f)$ are even and odd, respectively, we can also drop the Re before the integral for $e(t)$ and write

$$
e(t)=\int_{-\infty}^{\infty} I(f) Z(f) \epsilon^{j 2 \pi f t} d f
$$

Thus, the frequency-domain representations of $i(t)$ and $e(t)$ are, respectively, $I(f)$ and $I(f) Z(f)=E(f)$. Generally, then, the transfer function of a linear system is the ratio

$$
Z(f)=\frac{E(f)}{I(f)}
$$

where $E^{\prime}(f)$ is the frequency-domain representation of the output $e(t)$ caused by any inpul i( $(l)$ thal has a frequency-domain representation $I(f)$.

\section{3 Time Differentiation and Integration}

Let $v(t)$ have the frequency-domain representation $v(t)$; then

$$
v(t)=\int_{-\infty}^{m} V(f) \epsilon^{j 3 x f t} d f
$$

We differentiate with respect to time:

$$
\frac{d v(t)}{d t}=\int_{-\infty}^{\infty}[j 2 \pi f V(f)] \epsilon^{j 2 \pi f t} d t
$$

Hence, the frequency-domain representation of $\mathrm{dv} / \mathrm{dt}$ is $j 2 \pi \mathrm{fV}(\mathbf{f})$; $i$. e., differentiation in the time domain corresponds to multiplication by $j 2 \pi f$ in the frequency domain. Therefore, a device with the transfer function $(j 2 \pi f)^{k}$ has the effect of differentiating the time history of input $k$ times. Now

$$
v(t)=\int_{-\infty}^{t} \frac{d v(\tau)}{d \tau} d \tau
$$

Since the frequency-domain representation of $\mathrm{dv} / \mathrm{dt}$ has been shown to be $j 2 \pi f V(f)$ and we defined $V(f)$ as the frequency-domain representation of $v(t)$, it follows that time integration as shown above corresponds to multiplication by $1 / \mathrm{j} 2 \pi \mathrm{f}$ in the frequency domain. Therefore, a device with the transfer function $1 /(j 2 \pi f)^{k}$ and input $i(t)$ has the output

$$
e(t)=\int_{-\infty}^{t} \int_{-\infty}^{t_{k}} \cdots \int_{-\infty}^{t_{3}} \int_{-\infty}^{t_{2}} i\left(t_{1}\right) d t_{1} d t_{2} \ldots d t_{k-1} d t_{k} .
$$

We assume, of course, that the frequency-domain representations exist and will give the time functions by transformation. 


\section{4 Reduction of the Number of Parameters}

The transmission transfer function is

$$
T(f)=T_{0}(f) \epsilon^{-j(2 \pi r / c) E}(v / m) / v
$$

where

$$
T_{0}(f)=\frac{j \zeta_{0} f}{c r} \frac{h_{e}(f)}{Z_{0}(f)+z_{k}}(v / m) / v
$$

as given by Equation (33). The exponential factor $\epsilon^{-j(2 \pi \mathrm{r} / \mathrm{c}) \mathrm{f}}$ provides a lag of $\mathrm{r} / \mathrm{c}$ seconds in the time domain; this is the delay caused by a travel of $r$ meters. We will, then, consider only $T_{0}(f)$. Now, $h_{e}(f)$ and $Z_{o}(f)$ depend on a and $f$ only. in the product af as shown by E(quations (8), (9), and (18). (Note that $k \equiv 2 \pi / \lambda=2 \pi f / c_{+}$) Thus, for the case where we take $Z_{k}=Z_{c}$, where $Z_{c}$ does not depend on either $a$ or $f$, we have that $T_{0}(f)$ also depends on a and $f$ only in the product af. We can write, then,

$$
T_{\text {on }}(a f) \equiv T_{0}(f)
$$

The exciting pulses of either type depend on $t$ and $t_{w}$ only in the ratio $t / t_{w}$. Hence, we can define

$$
v_{g n}\left(t / t_{w}\right) \equiv v_{g}(t)
$$

Then

$$
\begin{aligned}
V_{g}(f) & =\int_{-\infty}^{\infty} v_{g}(t) t^{-j 2 \pi f t} d t \\
& =\int_{-\infty}^{\infty} v_{g n}\left(t / t_{w}\right) \epsilon^{-j 2 \pi f t} d t \\
& =t_{w} \int_{-\infty}^{\infty} v_{g n}(\tau) \epsilon^{-j 2 \pi t_{w} f \tau} d \tau \\
& =t_{w} V_{g n}\left(t_{w} f\right)
\end{aligned}
$$

where

$$
V_{\mathbf{g n}}(\nu)=\int_{-\infty}^{\infty} v_{\mathbf{g n}}(\tau) \epsilon^{-j 2 \pi \nu \pi} \mathrm{d} \tau
$$




$$
\begin{aligned}
e^{r a d}(t+r / c) & =\int_{-\infty}^{\infty} V_{g}(f) T_{o}(f) \epsilon^{j 2 \pi f t .} d f \\
& =\int_{-\infty}^{\infty} t_{w} V_{g n}\left(t_{w} f\right) T_{o n}(a f) \epsilon^{j 2 \pi f t} d f \\
& =\int_{-\infty}^{\infty} V_{g n}(\nu) T_{o n}\left(\frac{a}{t_{w}} \nu\right) e^{j 2 \pi \nu t / t_{w}} d \nu .
\end{aligned}
$$

The last integral above is a function only of $t / t_{w}$ and of $a / t_{w}$. Hence, $e^{r a d}(t+r / c)$ can be plotted against the time variable $l / t_{w}$ with thu $r a l i v a / t_{w}$ no a parameter. This eliminates the need for carrying $t_{w}$ as a separate parameter.

The rcoeption transfer function is

$$
S(f)=\frac{-H_{c}(f) Z_{L}(f)}{Z_{0}(f)+Z_{L}(f)} v /(v / m)
$$

which we rewrite as

$$
\frac{S(f)}{a}=\frac{-f e^{(f) Z_{c}}}{a f\left[Z_{o}(f)+7_{c}\right]} \frac{v /(v / m)}{I I}
$$

where we have taken $Z_{L}(f)=Z_{c}$. Clearly, for the reasons given above, $(1 / a) S(f)$ depends on a and $f$ only in the product af. We will call this the "reception transfer funcliun vei unit of antonna length.". We now define

$$
S_{n}(a f)=(1 / a) S(f)
$$

As in the transmissinn rase, we can write

$$
e_{n}^{i n c}\left(t / t_{w}\right) \equiv e^{l n c}(t)
$$

Then

$$
\begin{aligned}
E^{i n c}(f) & =\int_{--}^{\infty} e^{i n c}(t) \epsilon^{-j 2 \pi f t} d t \\
& =t_{w} E_{n}^{i n c}\left(t_{w} f\right)
\end{aligned}
$$

where

$$
E_{n}^{i n c}(\nu)=\int_{-\infty}^{\infty} e_{n}^{i n c}(\tau) \epsilon^{-j 2 \pi \nu \tau} d \tau
$$


Now

$$
\begin{aligned}
v_{L}(t) & =\int_{-\infty}^{\infty} E^{i n c}(f) S(f) \epsilon^{j 2 \pi f t} d f \\
& =\int_{-\infty}^{\infty} t_{w} E_{n}^{i n c}\left(t_{w} f\right) a S_{n}(a f) \epsilon^{j 2 \pi f t} d f \\
& =a \int_{-\infty}^{\infty} E_{n}^{i n c}(\nu) S_{n}\left(\frac{a}{t_{w}} \nu\right) \epsilon^{j 2 \pi \nu t / t_{w}} d \nu
\end{aligned}
$$

so that

$$
\frac{v_{L}(t)}{t_{w}}-\frac{a}{t_{w}} \int_{-\infty}^{\infty} E_{n}^{i n r}(\nu) s_{n}\left(\frac{a}{t_{w}} \nu\right) \epsilon^{. j 2 \pi \nu t / t_{w}} d \nu .
$$

Hence, we can plot $\left(1 / t_{w}\right) v_{L}(t)$ against the time variable $t / t_{w}$ with the ratio a/t ${ }_{w}$ as a parameter. This case differs from the transmission case in that the output itself is divided by the pulse width $t_{w}$.

We note that in both cases the elimination of the parameter $t_{w}$ requires that $Z_{k}$ or $Z_{\mathcal{L}}(f)$ not be dependent on $f$. Wherever $f$ appears, we must have it in the product af if we wish to eliminate $t_{w}$ as a separate parameter.

\section{5 Excitation Pulses}

The excitation pulses used in transmission are:

Gaussian--

$$
v_{g}(t)=\epsilon^{-t^{2} / 2 t_{1}^{2}} \text { volt }
$$

where

$$
t_{1}=0.4246 t_{w} \mathrm{sec}
$$

which has the frequency-domain representation

$$
V_{g}(f)=\frac{1}{\sqrt{2 \pi} f_{1}} \epsilon^{-f^{2} / 2 f_{1}^{2}} \text { volt } / H z
$$

where

$$
f_{1}=1 / 2 \pi t_{1} \mathrm{~Hz}
$$


almost-rectangular--

$$
v_{g}(t)=\int_{-20 / \pi t_{w}}^{20 / \pi t_{w}} \frac{\sin \pi t_{w} f}{\pi f} \epsilon^{j 2 \pi f t} d f \text { volt }
$$

which has the frequency-domain representation

$$
\begin{array}{rlrl}
V_{g}(f) & =\frac{\sin \pi t_{w} f}{\pi f} v / H z, & f \mid<20 / \pi t_{w} H z \\
& =0 & & |f|>20 / \pi t_{w} H z .
\end{array}
$$

The expression ( $\left.\sin \pi \mathrm{t}_{w} f\right) \pi f$ is the frequency-domain representation of a reclangulid. pulse of unit amplitude and width $t_{w}$ sec. Hence, $v_{g}(t)$ results from truncating the spectrum of inis reclanyular. pulse at the frequency $20 / \pi t_{w} \mathrm{~Hz}$. Only the Gaussian pulse is used in reception, i.e.,

$$
\varphi^{i n c}(t)=e^{-t^{2} / 2 t_{1}^{2}} \text { volt } / \text { meter }
$$

where

$$
t_{1}=0.4246 t_{w} \sec
$$

which has the frequency-domain representation

$$
E^{i n c}(f)=\frac{1}{\sqrt{2 \pi} f_{1}} \epsilon^{-f^{2} / 2 f_{1}^{2}}(v / m) / H z
$$

where

$$
f_{1}=1 / 2 \pi t_{1} \mathrm{~Hz} \text {. }
$$

\section{6 Limiting Value of $\beta / \alpha$}

We will show below that

$$
\sum_{n=1,3,5, \ldots}^{\infty} \frac{2 n+1}{n(n+1)}\left[P_{n}\left(\cos \theta_{0}\right)\right]^{2}=2 n \cot \left(\theta_{0} / 2\right)
$$

and that

$$
\lim _{k a \rightarrow \infty} \zeta_{n}(k a)=j
$$


These two results give

$$
\begin{aligned}
\lim _{k a \rightarrow \infty}\left\{\frac{j \zeta_{0}}{2 \pi Z_{c}} \sum_{n=1,3,5, \ldots}^{\infty} \frac{2 n+1}{n(n+1)}\left[P_{n}\left(\cos \theta_{0}\right)\right]^{2} \zeta_{n}(k a)\right. & =\frac{-\zeta_{0}}{2 \pi Z_{c}} \ell n \cot \left(\theta_{0} / 2\right) \\
& =-1
\end{aligned}
$$

where the last step follows from the definition of $Z_{c}$ given by Equation (7). We substitute the latter result into Equation (9) to obtain

$$
\lim _{\mathbf{k a \rightarrow \infty}}(\beta / \alpha)=0
$$

We now show the first result above. We have the inentity ${ }^{21}$

$$
\sum_{n=1}^{\infty} \frac{2 n+1}{n(n+1)} P_{n}(x) P_{n}(y)=2 \text { in } 2-1-i n[(1-x)(1+y)], \quad-1<x \leq y<1 .
$$

We replace $x$ by $-x$ in the series above. With $P_{n}(-x)=(-1)^{n} P_{n}(x)$, we now have

$$
\sum_{n=1}^{\infty} \frac{2 n+1}{n(n+1)}(-1)^{n} P_{n}(x) P_{n}(y)=2 \ln 2-1-\ell n[(1+x)(1+y)], \quad-1<-x \leq y<1
$$

We subtract the second identity froin the first to obtain

$$
2 \sum_{n=1,3,5, \ldots}^{\infty} \frac{2 n+1}{n(n+1)} P_{n}(x) P_{n}(y)=\ell n\left[\frac{1+x}{1-x}\right], \quad 0 \leq|x| \leq y<1
$$

where the condition on $x$ and $y$ results from combining the two conditions above. Let us take $\mathrm{x}=\mathrm{y}=\cos \theta_{0}$, where $0<\theta_{0}<\pi / 2$. We then have the desired result

$$
2 \sum_{n=1,3,5, \ldots}^{\infty} \frac{2 n+1}{n(n+1)}\left[P_{n}\left(\cos \theta_{0}\right)\right]^{2}=2 \cdot h n \cot \left(\theta_{0} / 2\right)
$$

where we have used the identity

$$
\left[\frac{1-\cos \theta_{0}}{1+\cos \theta_{0}}\right]^{\frac{1}{2}}=\tan \left(\theta_{0} / 2\right) .
$$

We now show that $\zeta_{n}(k a) \rightarrow j$ as $k a \rightarrow \infty$. We have ${ }^{12}$

$$
h_{n}^{(2)}(z)=\sqrt{\pi / 2 z} H_{n+\frac{1}{2}}^{(2)}(z) .
$$


But, when $|z|>>1,|z| \gg|\nu|^{2}$, and $-1 / 2 \leq$ phase $z \leq \pi / 2,{ }^{13}$

$$
\mathrm{H}_{\nu}^{(2)}(\mathrm{z}) \doteq \sqrt{2 / \pi \mathrm{z}} \epsilon^{-j(z-\pi / 4-\nu \pi / 2)}
$$

Hence,

$$
\begin{aligned}
h_{n}^{(2)}(z) & =(1 / z) \epsilon^{-j(z-\pi / 2-n \pi / 2)} \\
& =\left(j^{n+1} / z\right) \epsilon^{-j z} .
\end{aligned}
$$

Thus, from Equation (10)

$$
\zeta_{n}(z)=\frac{-1}{j+n / z} \text {. }
$$

Then,

$$
\lim _{z \rightarrow \infty} \zeta_{n}(z)=j
$$

\subsection{Effective Length of Long Antenina}

In Section 4.6 we developed the identity

$$
2 \sum_{n=1,3,5, \ldots}^{\infty} \frac{2 n+1}{n(n+1)} P_{n}(x) P_{n}(y)=\ell n\left[\frac{1+x}{1-x}\right], \quad 0 \leq|x| \leq y<1 .
$$

We differentiate with respect to $\mathrm{x}$ and divide by 2 to obtain

$$
\sum_{n=1,3,5, \ldots}^{\infty} \frac{2 n+1}{n(n+1)} P_{n}^{\prime}(x) P_{n}(y)=\frac{1}{1-x^{2}}, \quad 0 \leq|x| \leq y<1 .
$$

Now, from the approximation for $h_{n}^{(2)}\left(y^{\prime}\right)$ tor lur'gu a given in the latter part of Section 4.6, we have

$$
\begin{aligned}
h_{n-1}^{(2)}(z)-(n / z) h_{n}^{(2)}(z) & \doteq\left(j^{n} / z\right) \epsilon^{-j z}-(n / z)\left(j^{n+1} / z\right) \epsilon^{-j z} \\
& =\left(j^{n} / z\right) \epsilon^{-j z}(1-j n / z) \\
& =\left(j^{n} / z\right) \epsilon^{-j z} .
\end{aligned}
$$


We now take $\mathrm{z}=\mathrm{ka}$ and $\beta / \alpha=0$ and substitute into Equation (18) to obtain

$$
\begin{aligned}
\lim _{k a \rightarrow \infty} k h_{e}(f) & =\frac{1}{k a}\left(-\epsilon^{-j k a}\right) \sum_{n=1,3,5, \ldots}^{\infty} \frac{2 n+1}{n(n+1)} P_{n}\left(\cos \theta_{0}\right) P_{n}^{1}(0) \frac{j^{n+1}}{\left(j^{n} / k a\right) \epsilon^{-j k a}} \\
& =-j \sum_{n=1,3,5, \ldots}^{\frac{2 n+1}{n(n+1)} P_{n}\left(\cos \theta_{o}\right) P_{n}^{1}(0)} \\
& =-j
\end{aligned}
$$

where we have used the identity developed in the first part of this section with $x=0$ and $y=\cos \theta_{0}$, and have also used

$$
P_{n}^{\prime}(0)=\bar{P}_{n}^{1}(\overline{0})
$$

which follows from Equation (13). 


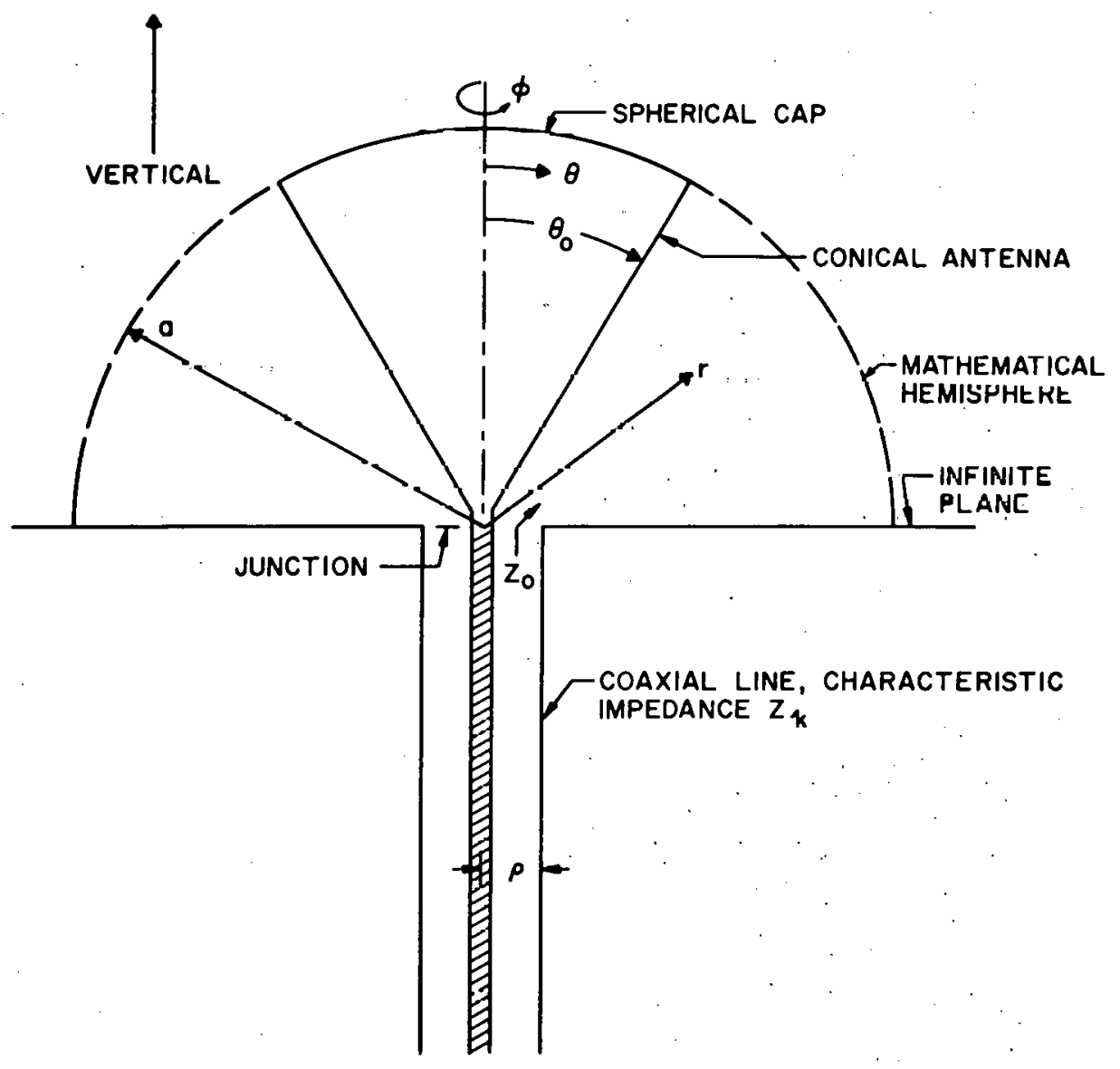

Fig. 1. Spherically-Capped, Conical Antenna Fed by Coaxial Feed Line 


\section{TRANSMISSION}

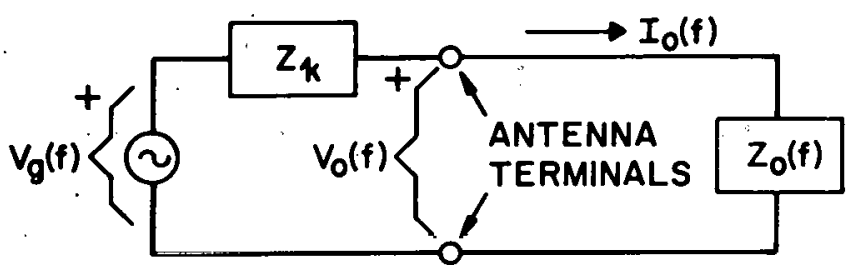

$$
Z_{k}=Z_{c} \equiv \lim _{a f \rightarrow \infty} Z_{o}(f)
$$

$V_{g}(f), V_{0}(f)$ AND $I_{0}(f)$ ARE FREQUENC' $r$-DOMAIN REPRESENTATIONS OF $v_{g}(t), v_{0}(t)$ AND $i_{0}(t)$

\section{RECEPTION}

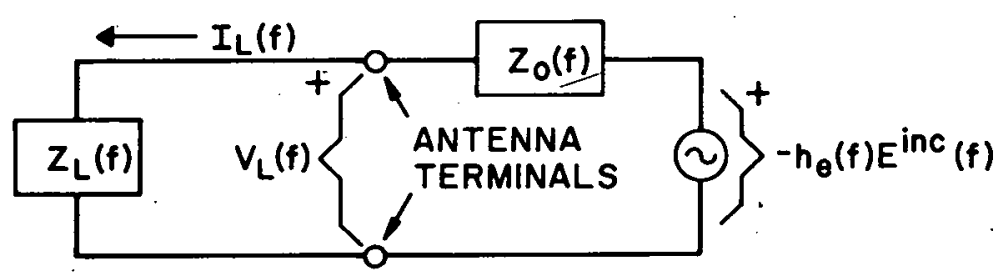

$E^{\text {inc }}(f), I_{L}(f)$ AND $V_{L}(f)$ ARE FREQUENCY-DOMAIN REPRESENTATIONS OF $e^{i n c}(t), i_{L}(t)$ AND $v_{L}(t)$.

Fig. 2. Equivalent Circuits for Transmission and Reception 


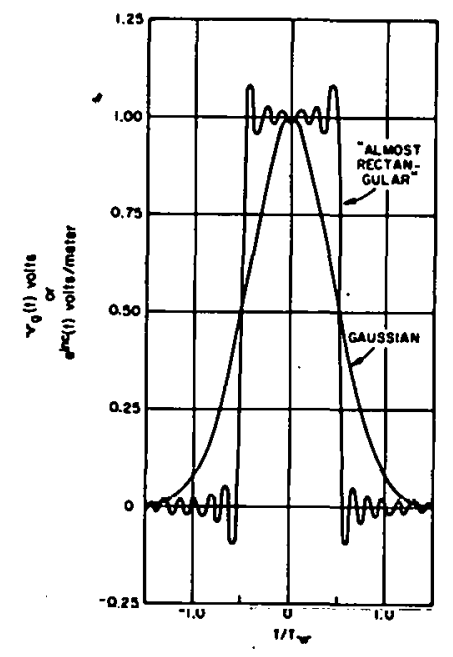

Fig. 3. The Two Excitation-Pulse Shapes

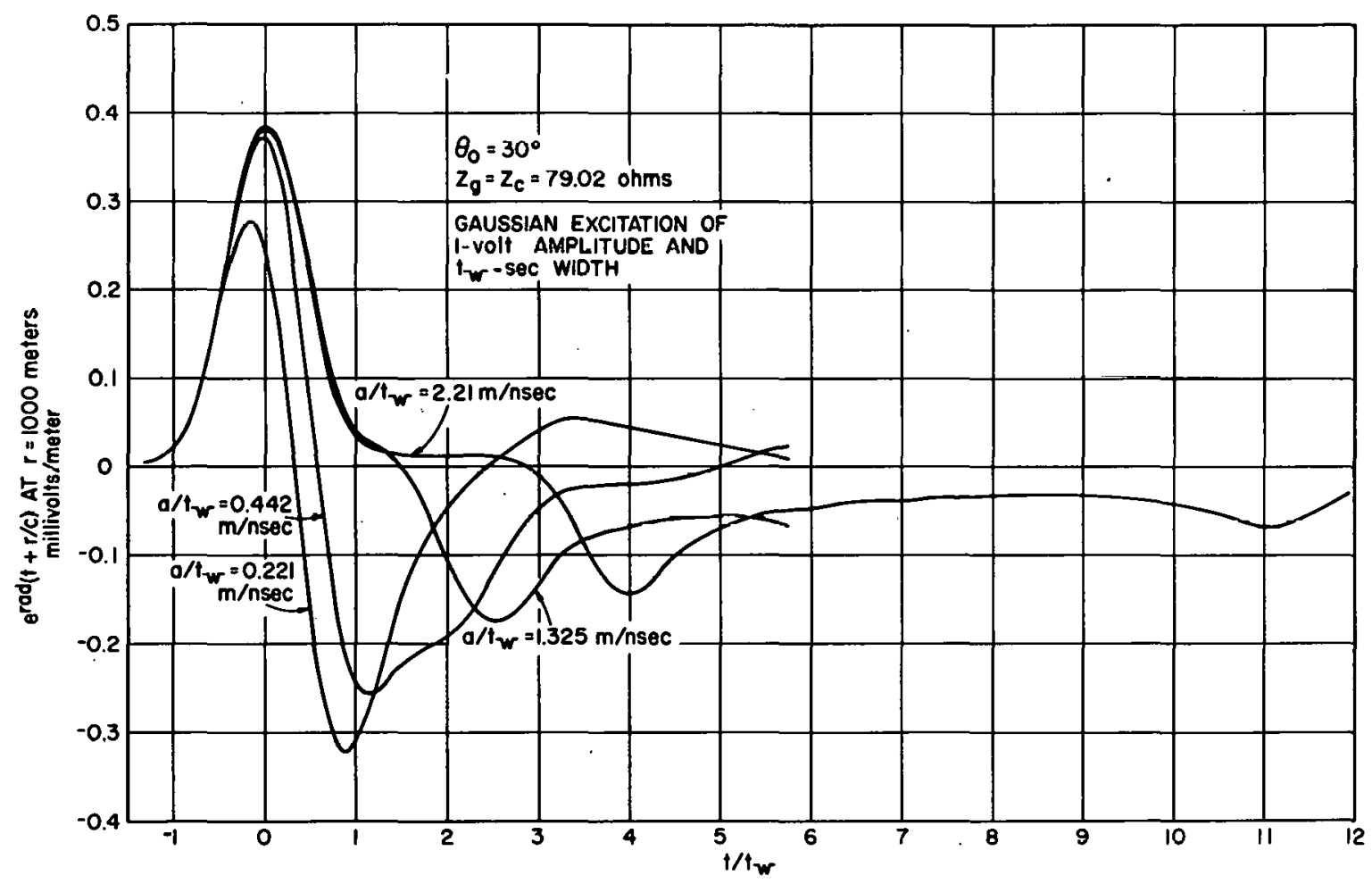

Fig. 4. The Time History of the Radiated Field in the Equatorial Plane at 1000 Meters From the Antenna, Translated $r / c=10^{-5} / 3 \mathrm{sec}$ to Ieft to Remove Delay due to 1000 Meters of Travel 


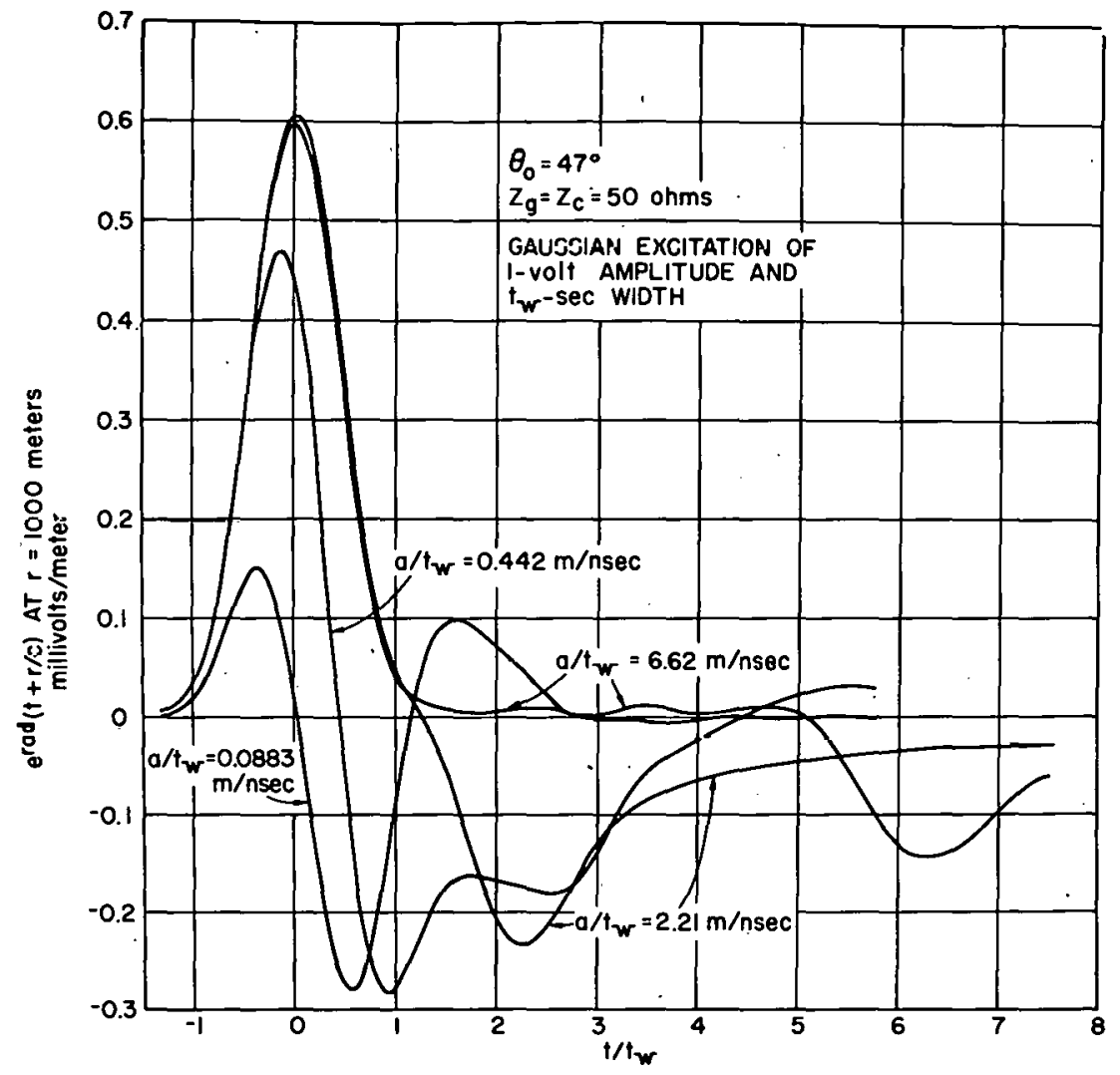

Fig. 5. The Time History of the Radiated Field in the Equatorial Plane at 1000 Meters From the Antenna, Translated $r / c=10^{-5} / 3 \mathrm{sec}$ to Left to Remove Delay due to 1000 Meters of Travel 


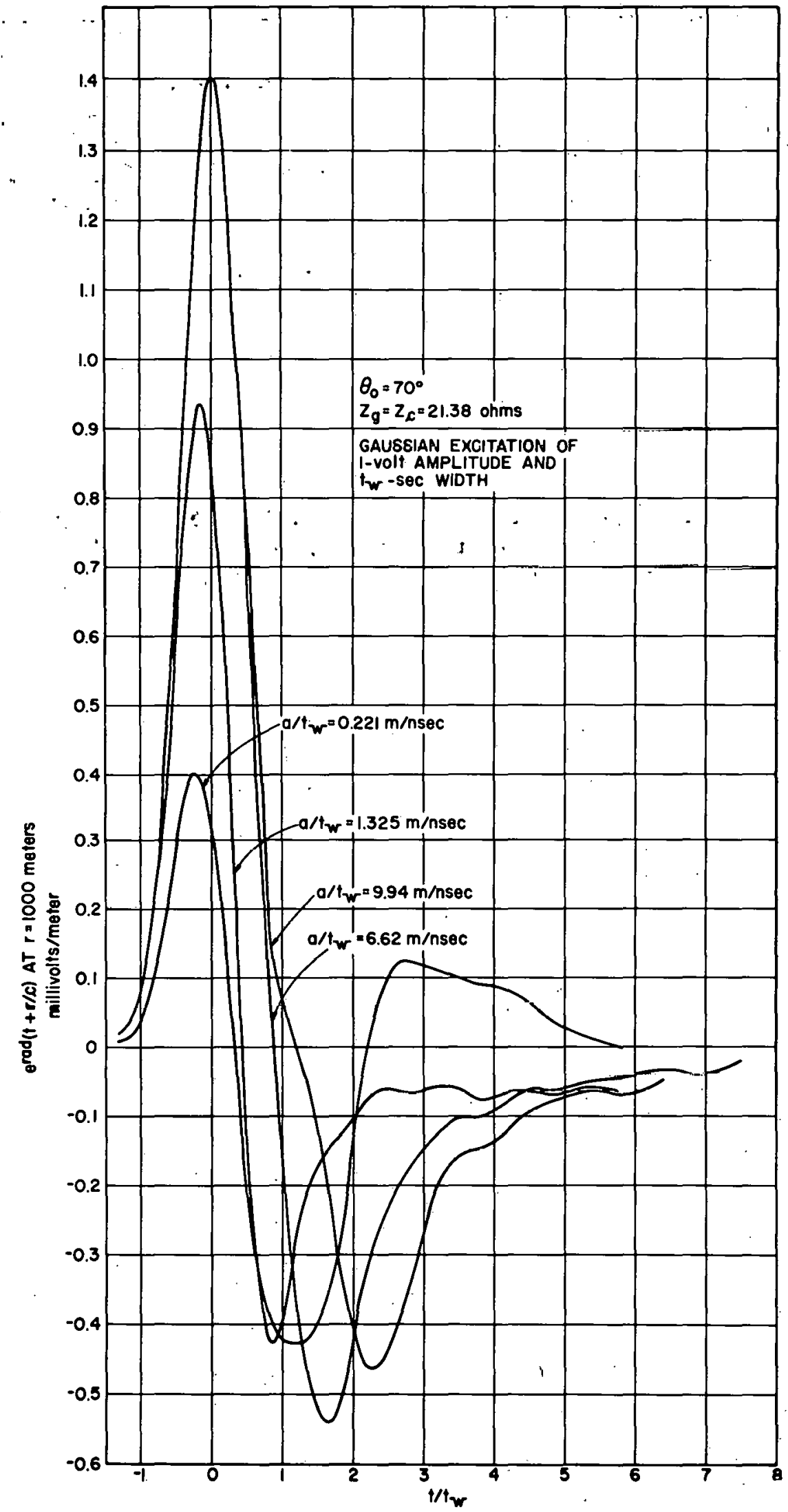

Fig.6. The Time History of the Radiated Field in the Equatorial Plane at 1000 Meters From the Antenna, Translated $r / c=10^{-5} / 3 \mathrm{sec}$ to Left to. Remove Delay due to 1000 Meters of Travel 
(7)
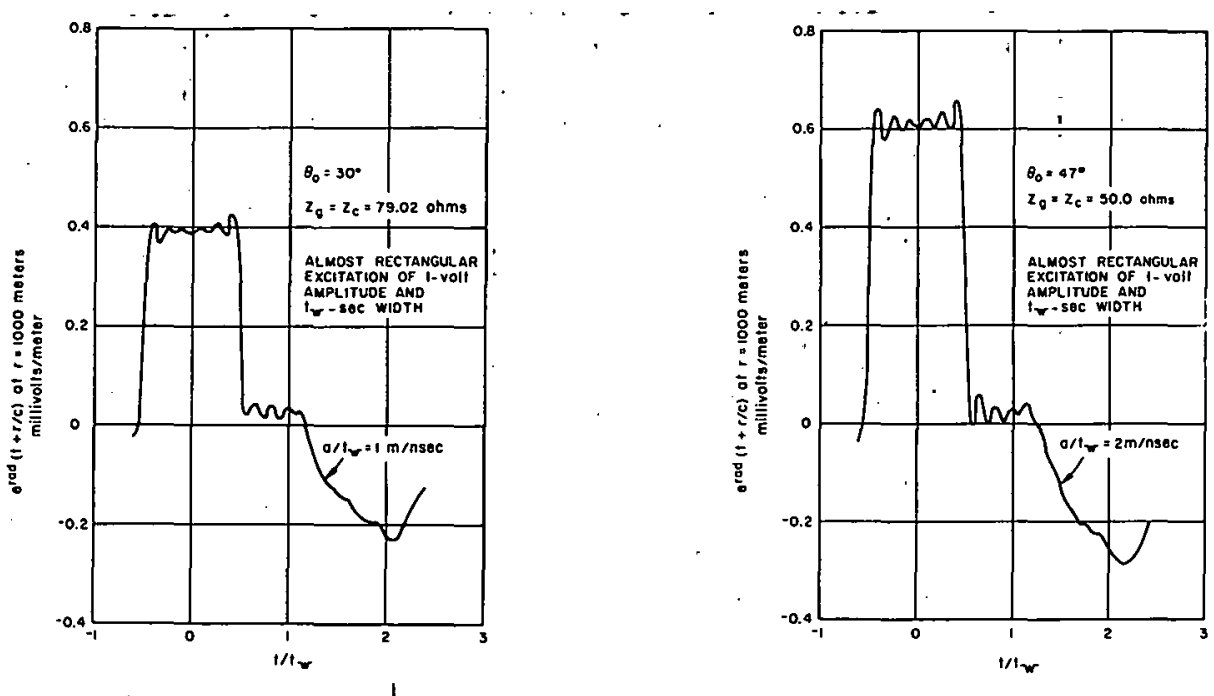

(8)

Fig. 7. The Time History of the Radiated Field in the Equatorial Plane at and 1000 Meters From the Antenna, Translated $r / c=10^{-5} / 3 \mathrm{sec}$ to Left Fig. 8. to Remove Delay due to 1000 Meters of Travel

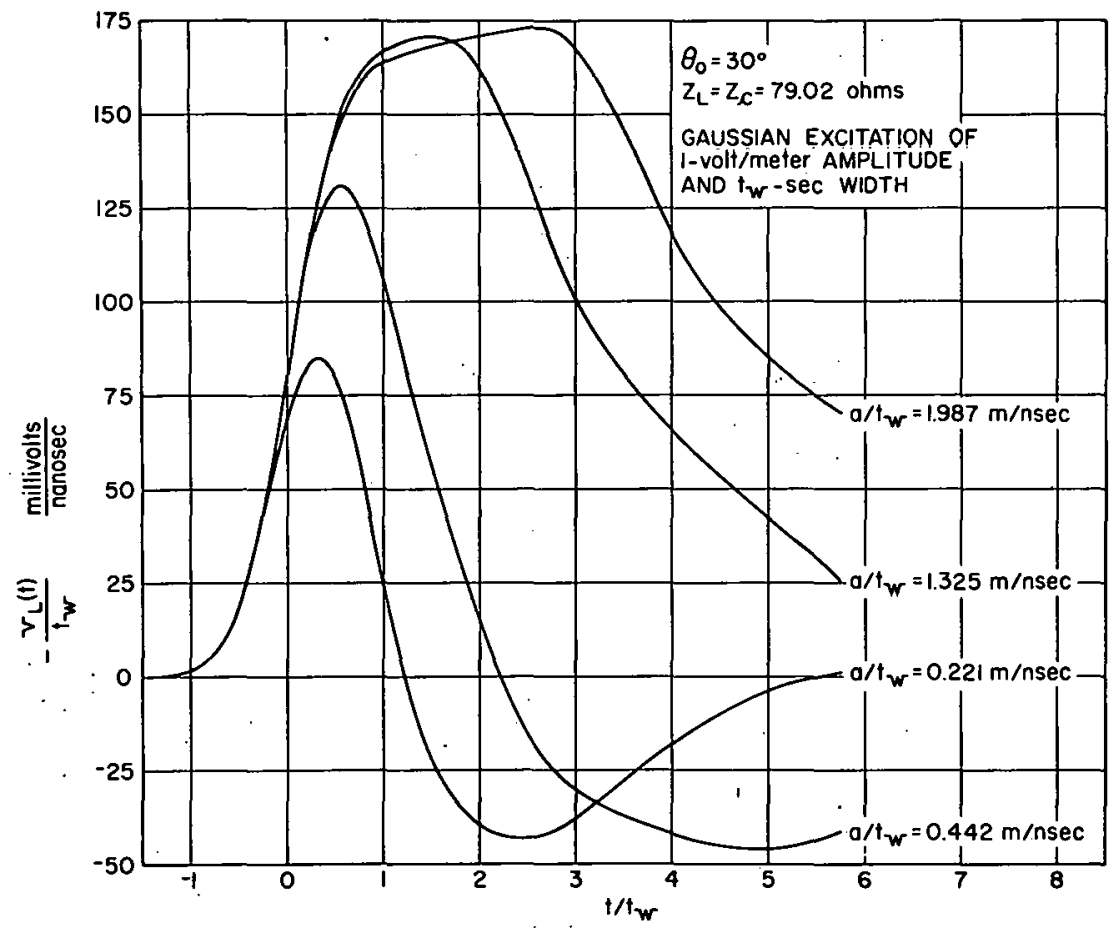

Fig. 9. The Time History of Voltage at Receiving Antenna Terminals. (Ordinate is Negative of Load Voltage Divided by Pulse Width.) 
(10)

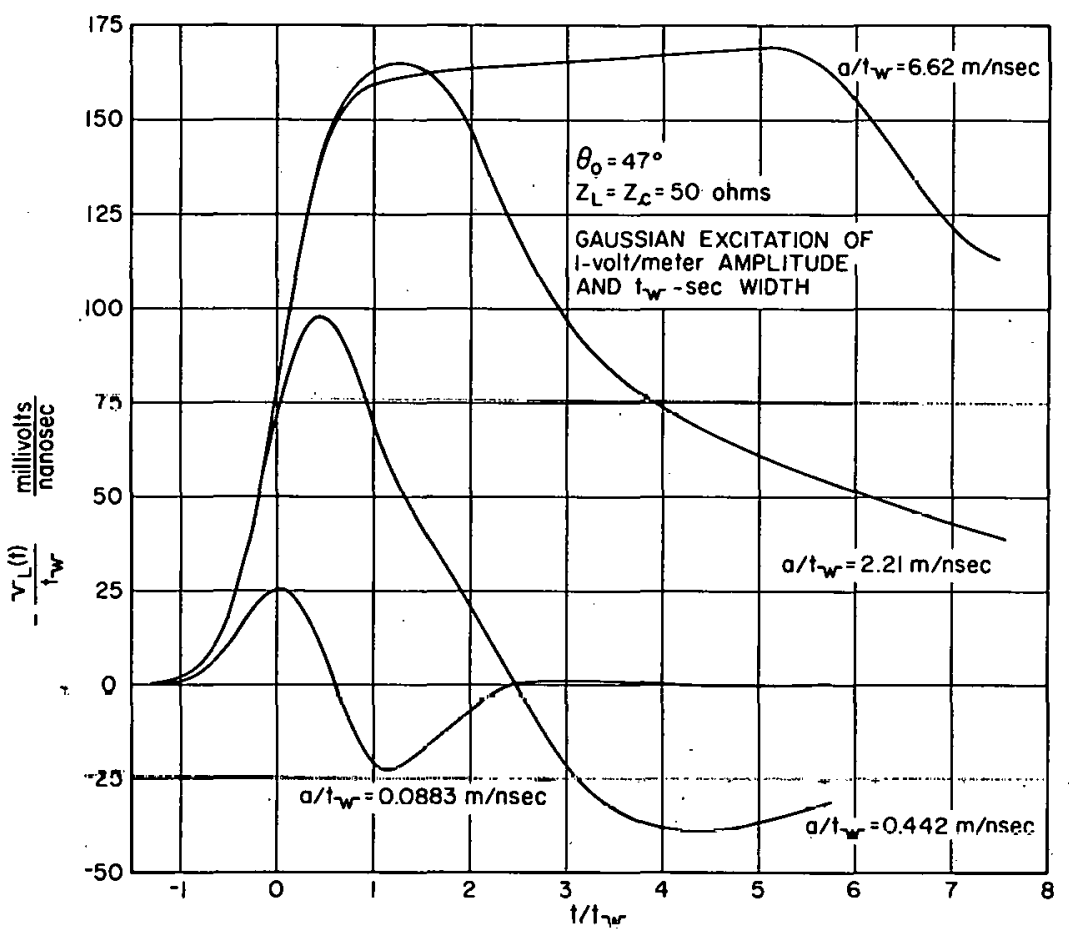

(11)

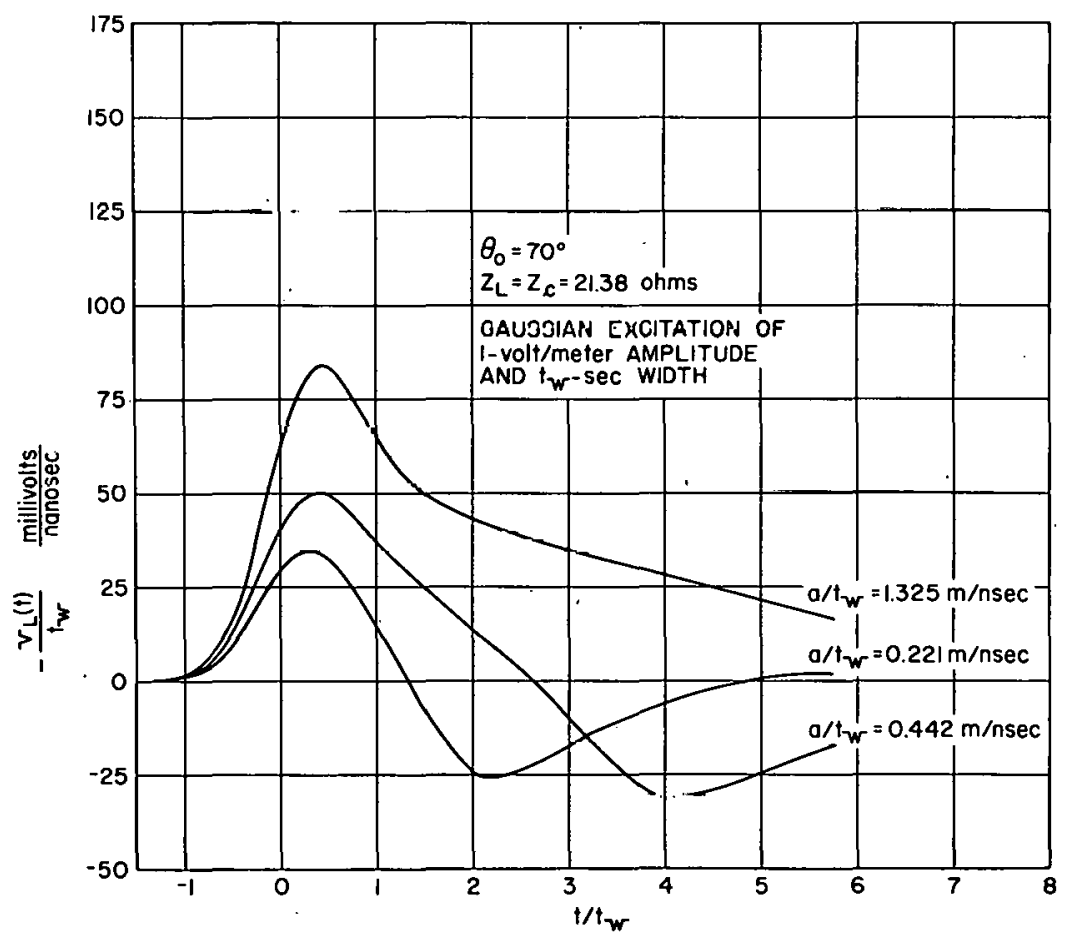

Fig. 10. The Time History of Voltage at Recelving Antenna Terminals. and

Fig. 1l. (Ordinate is Negative of Load Voltage Divided by Pulse Width.) 


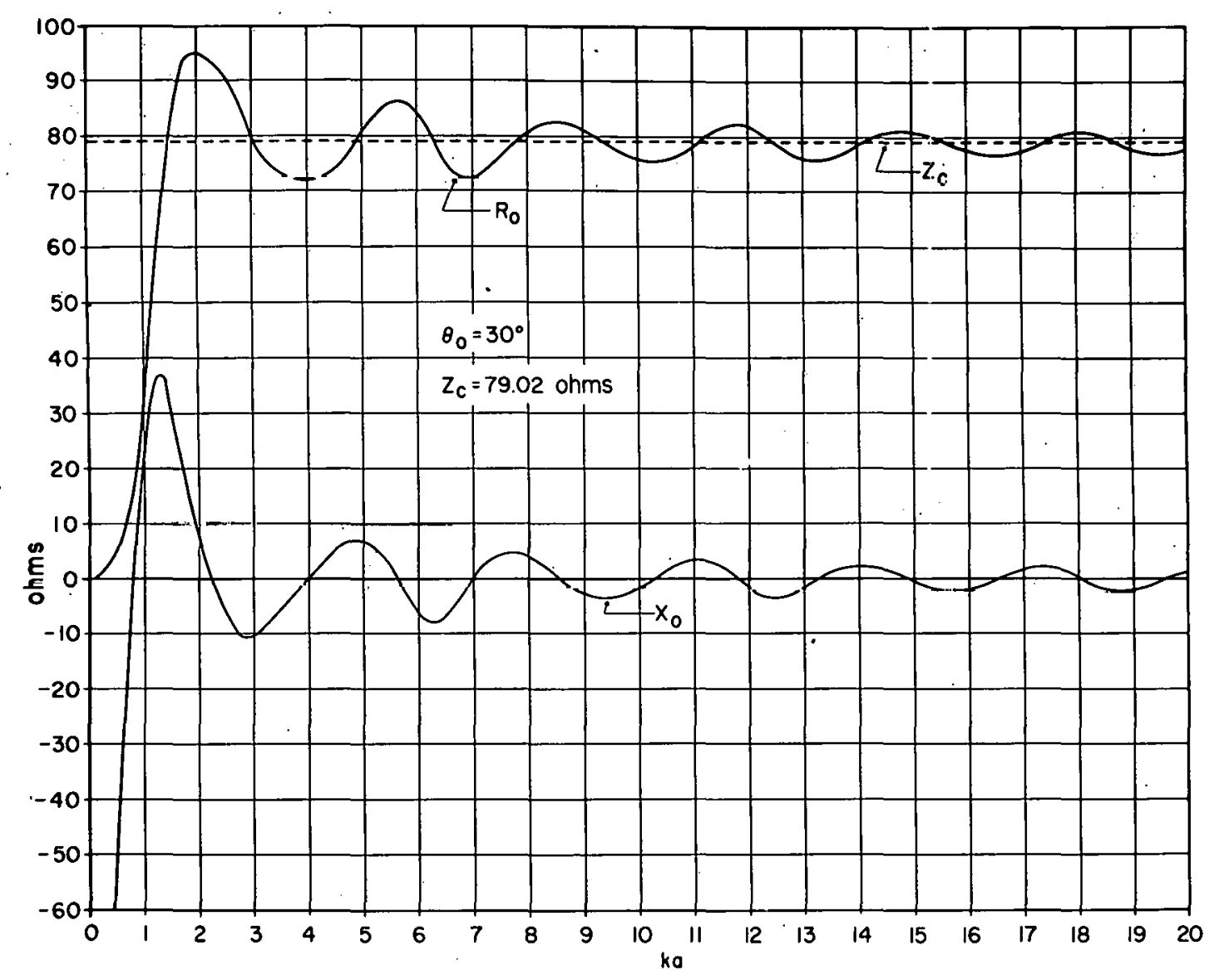

Fig. 12. Input Impedance $z_{\circ}=R_{\circ}+j x_{\circ}$ of Wide-Angle Conical Antennas 


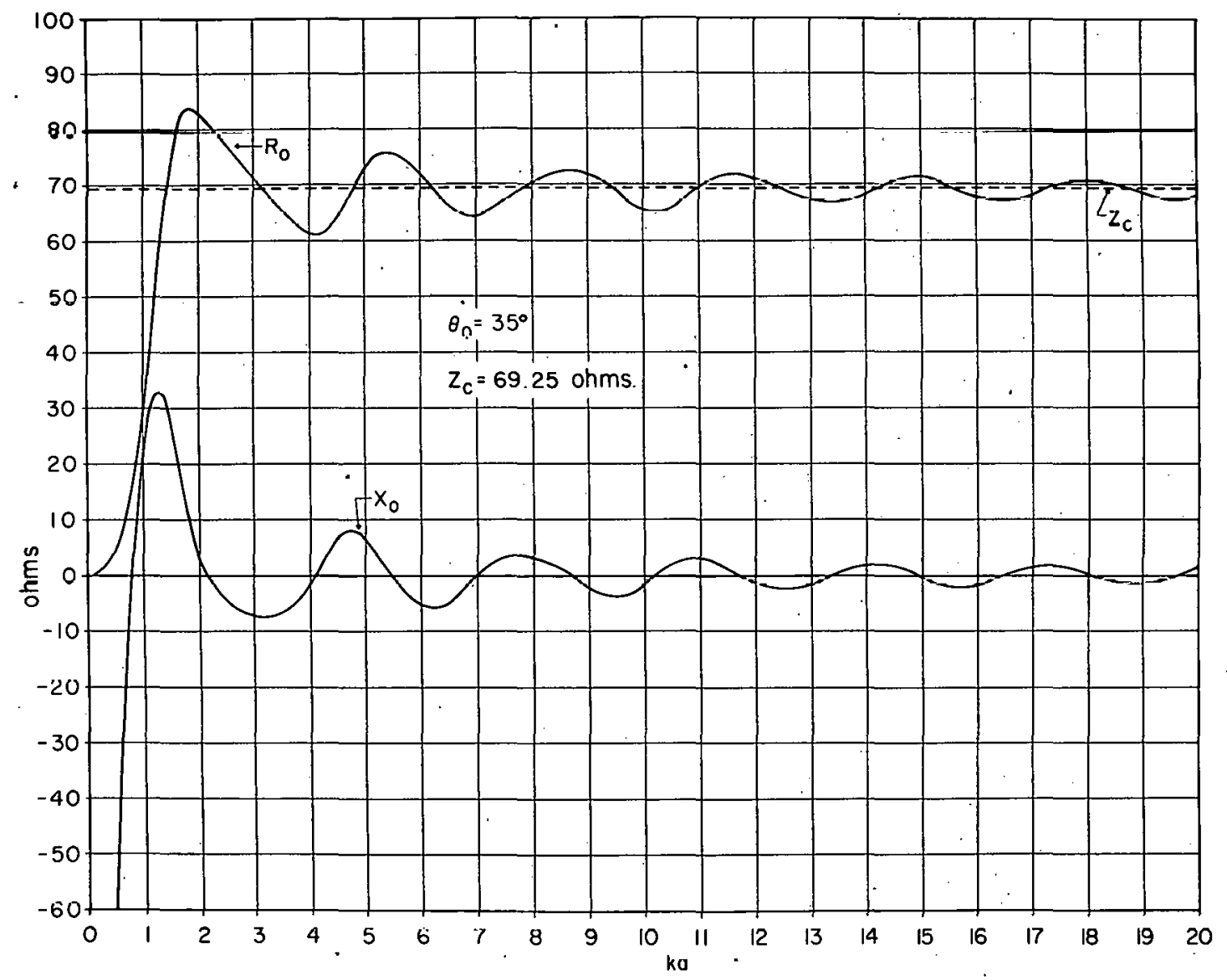

Fig. 13. Input Impedance $Z_{o}=R_{0}+j X_{0}$ of Wide-Angle Conical Antennas 


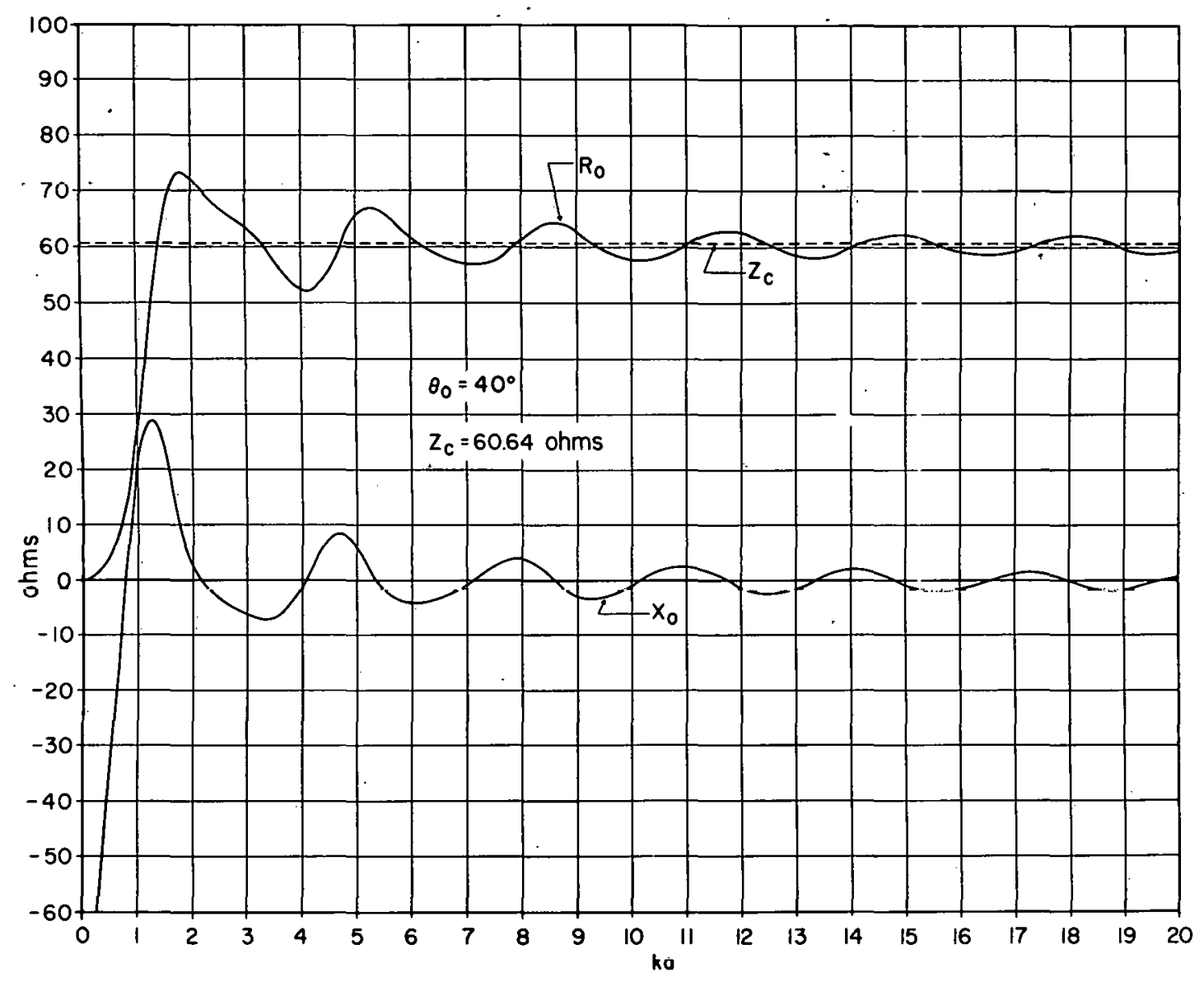

Fig. 14. Input Impedance $Z_{0}=R_{0}+j X_{0}$ of Wide-Angle Conical Antennas 


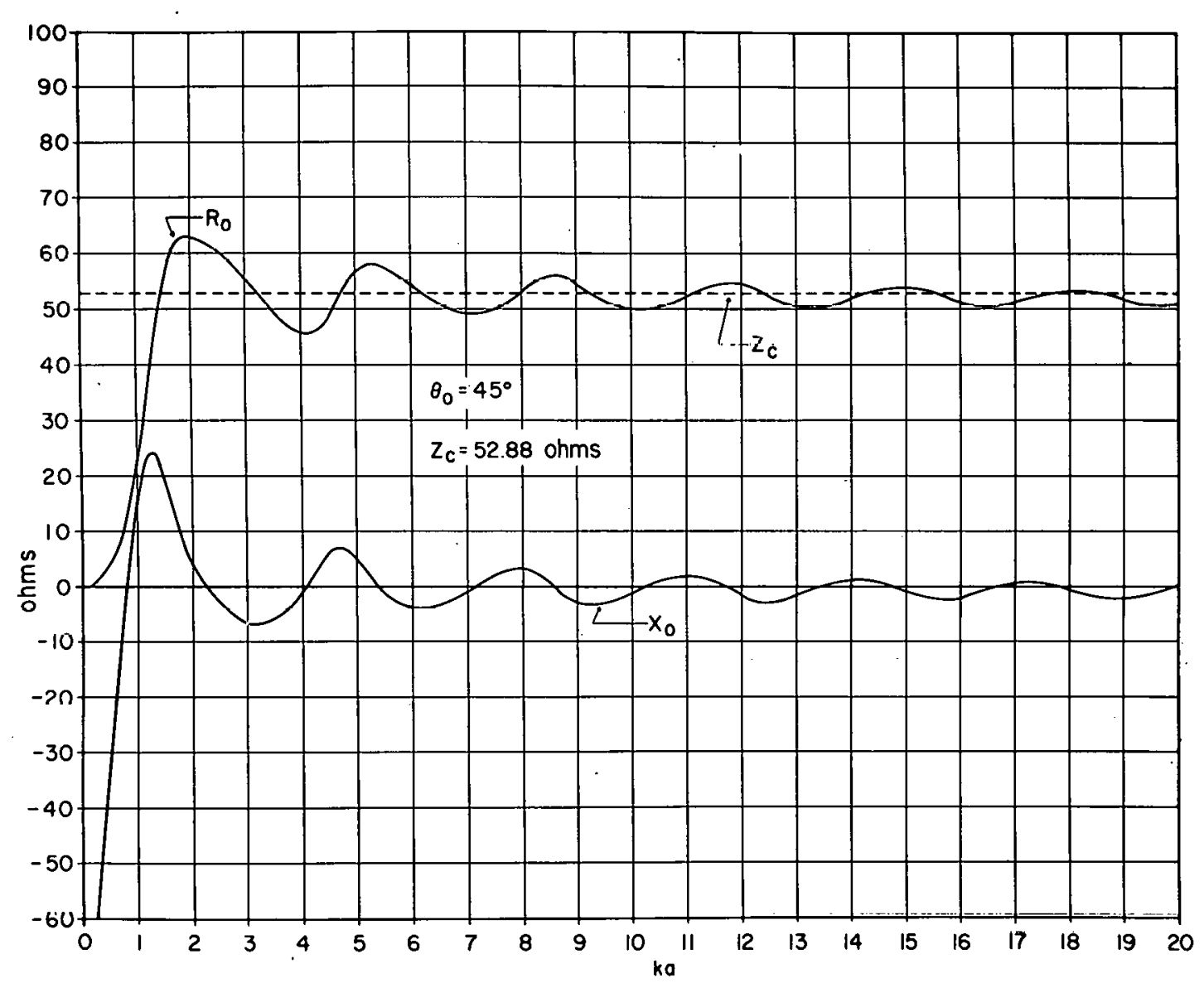

Fig. 15. Input Impedance $z_{0}=R_{0}+j x_{0}$ of Wide-Angle Conical Antennas 


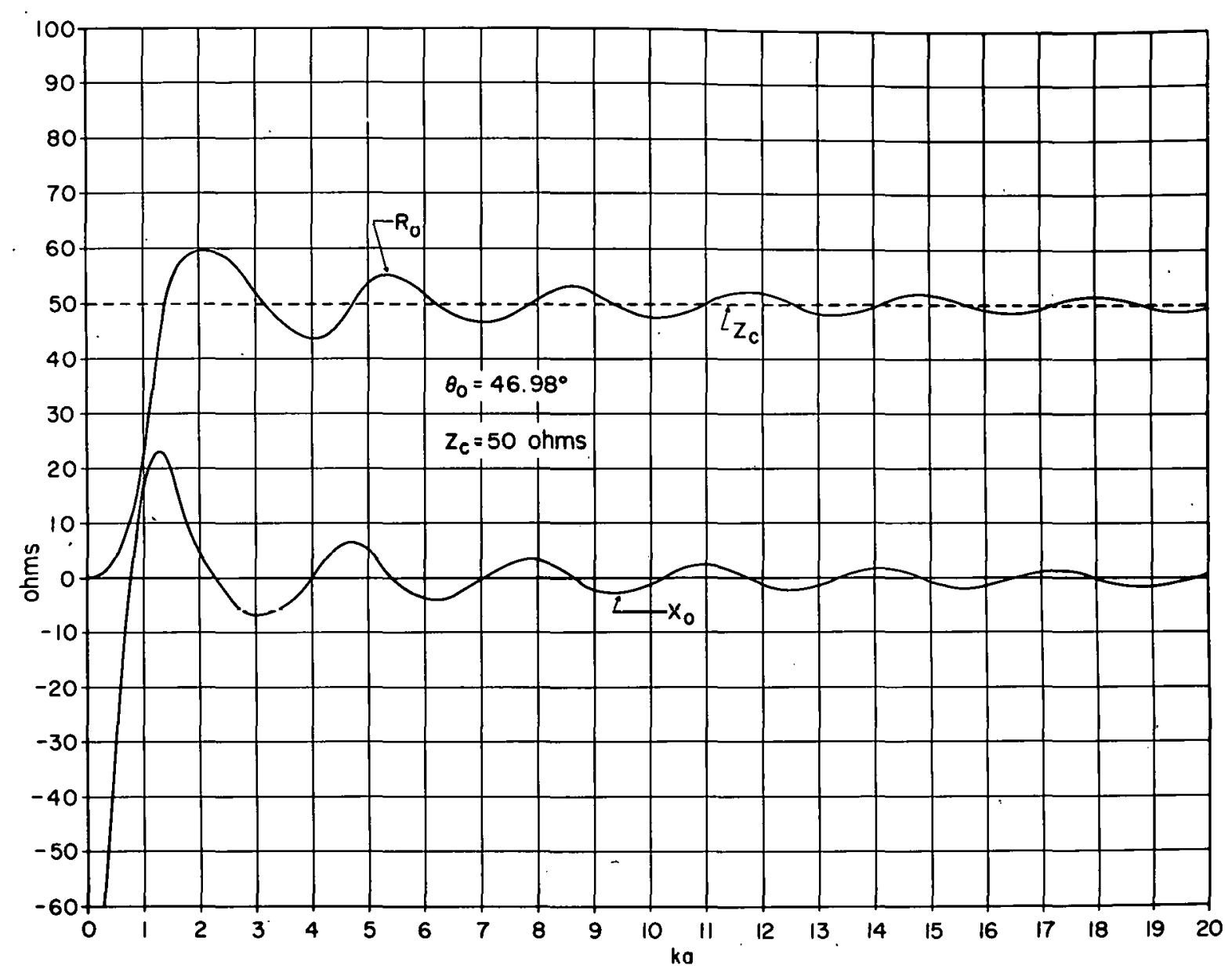

Fig. 16. Input Impedance $Z_{0}=R_{0}+j x_{0}$ of Wide-Angle Conical Antennas 


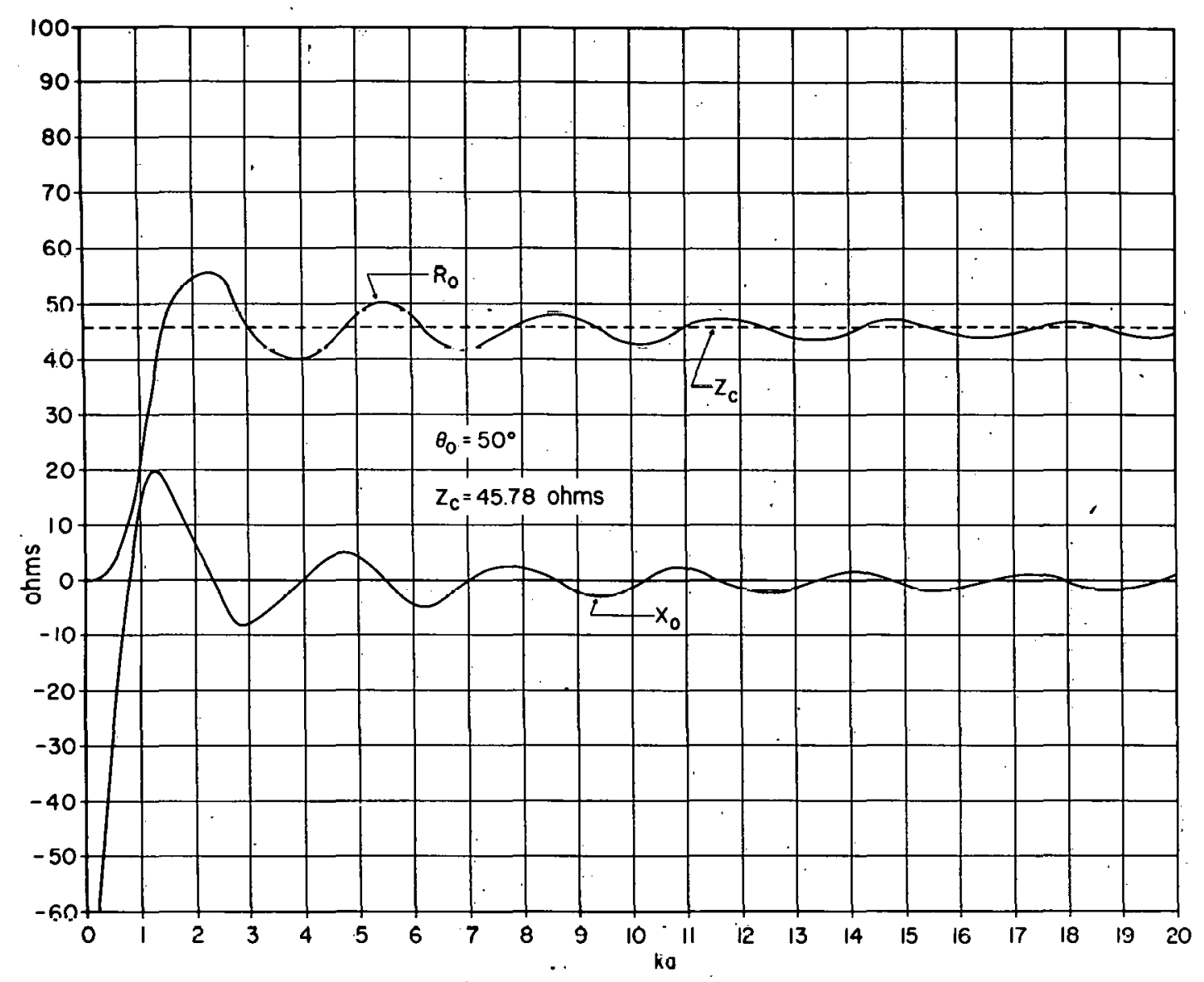

Fig. 17. Input Impedance $Z_{o}=R_{0}+j X_{0}$ of Wide-Angle Conical Antennas 


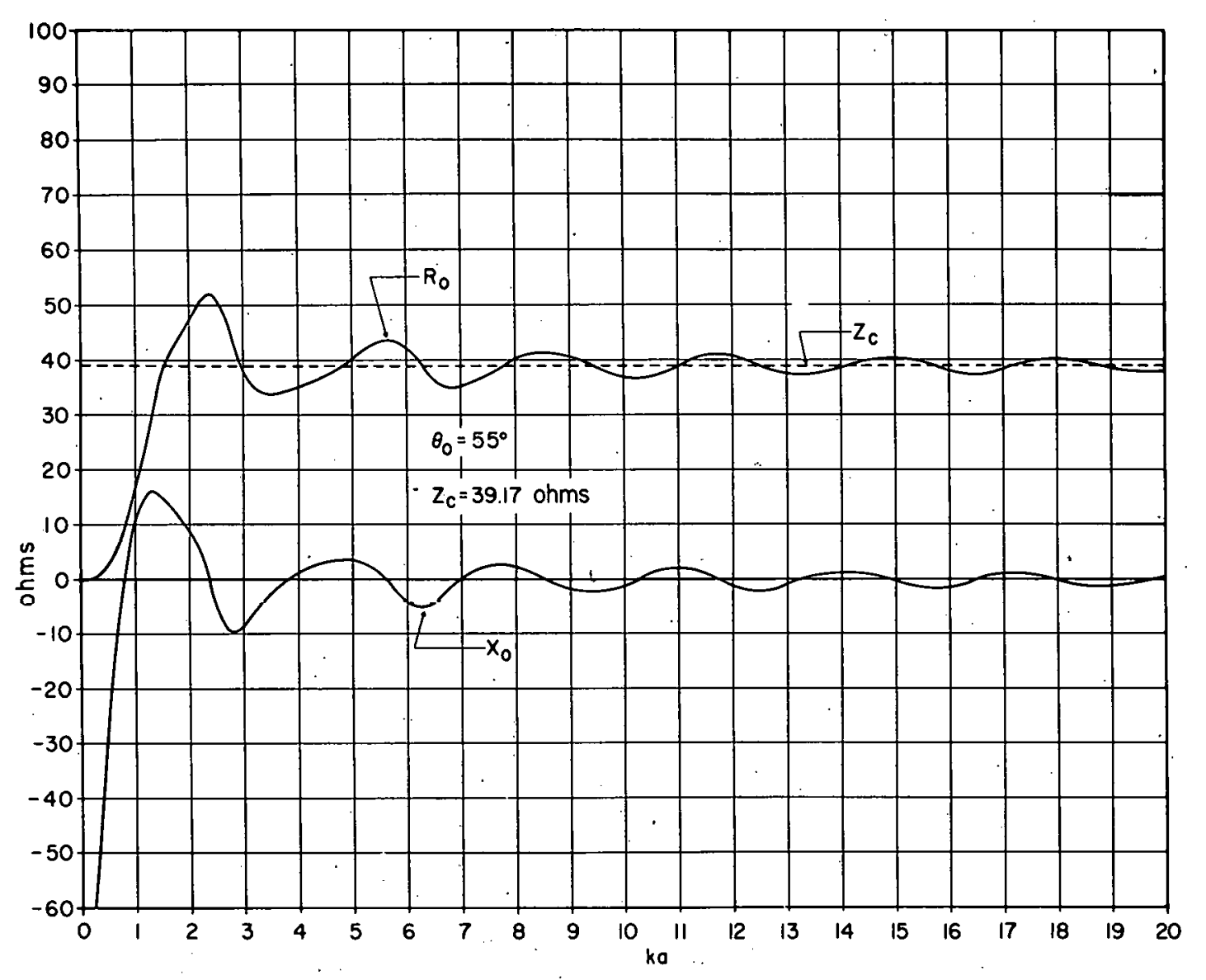

Fig. 18. Input Impedance $Z_{0}=R_{0}+j x_{0}$ of Wide-Angle Conical Antennas 


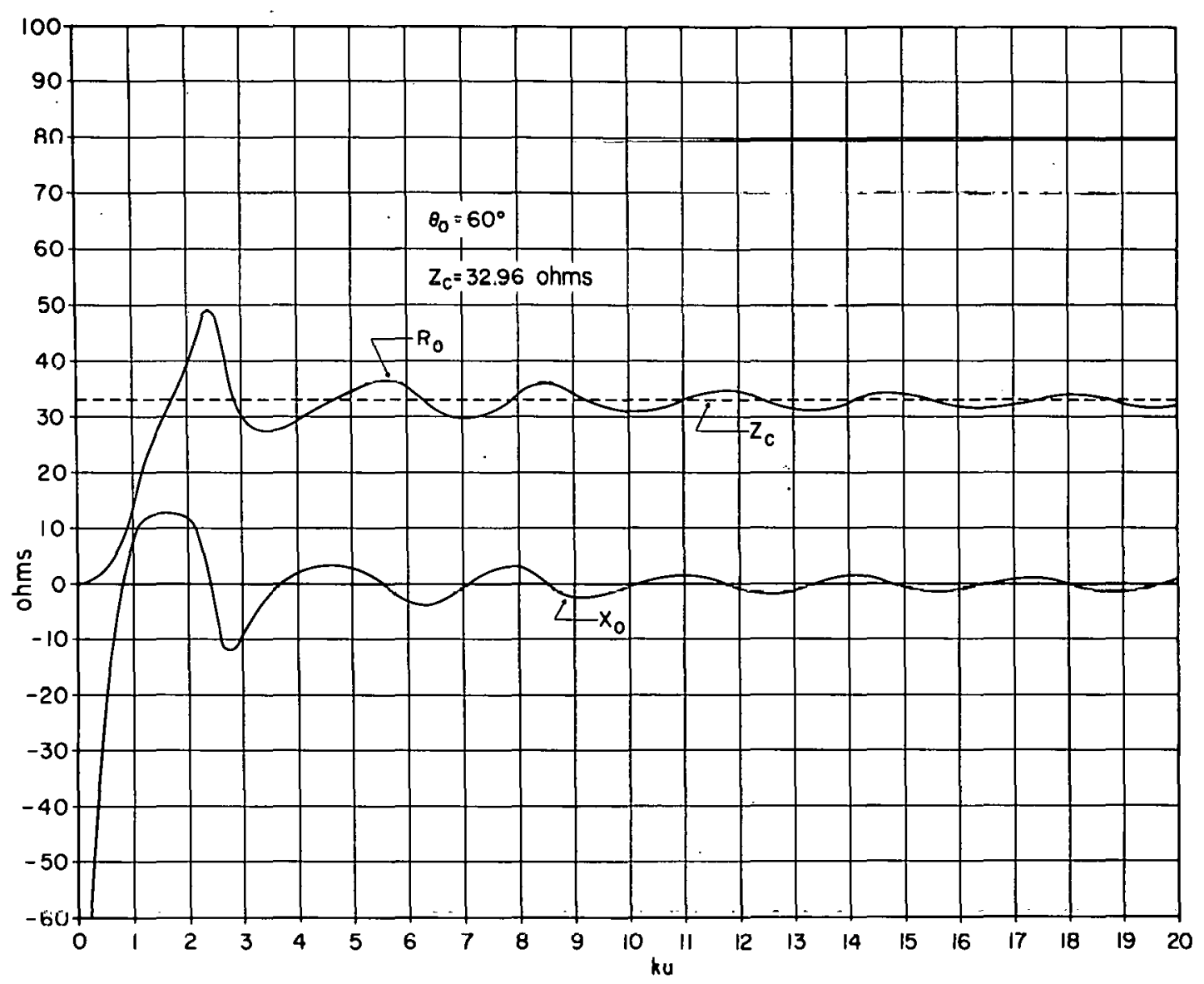

Fig. 19. Input Impedance $Z_{0}=R_{0}+j X_{0}$ of Wide-Angle Conical Antennas 


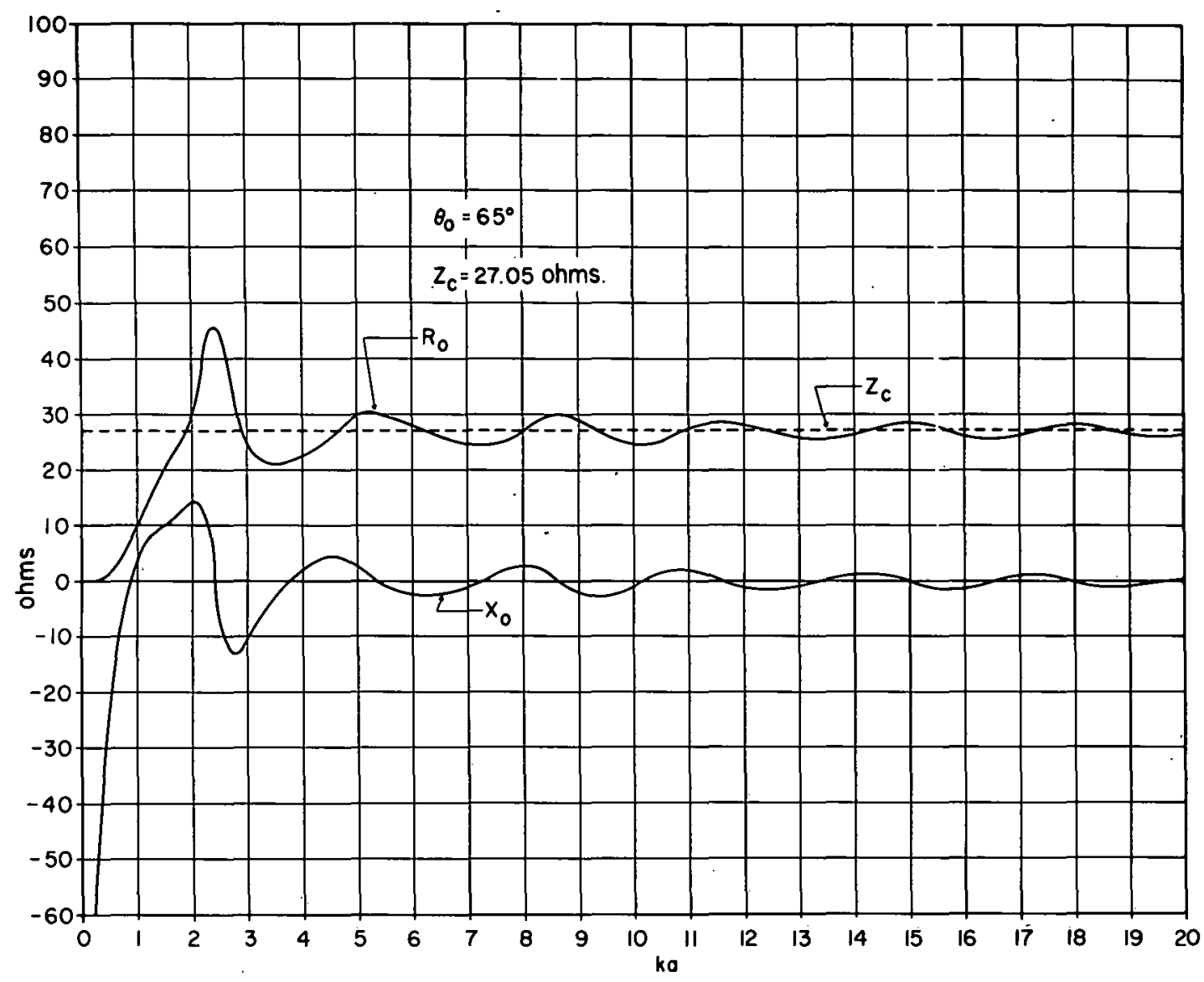

Fig. 20. Input Impedance $Z_{0}=R_{0}+j X_{0}$ of Wide-Angle Conical Antennas 


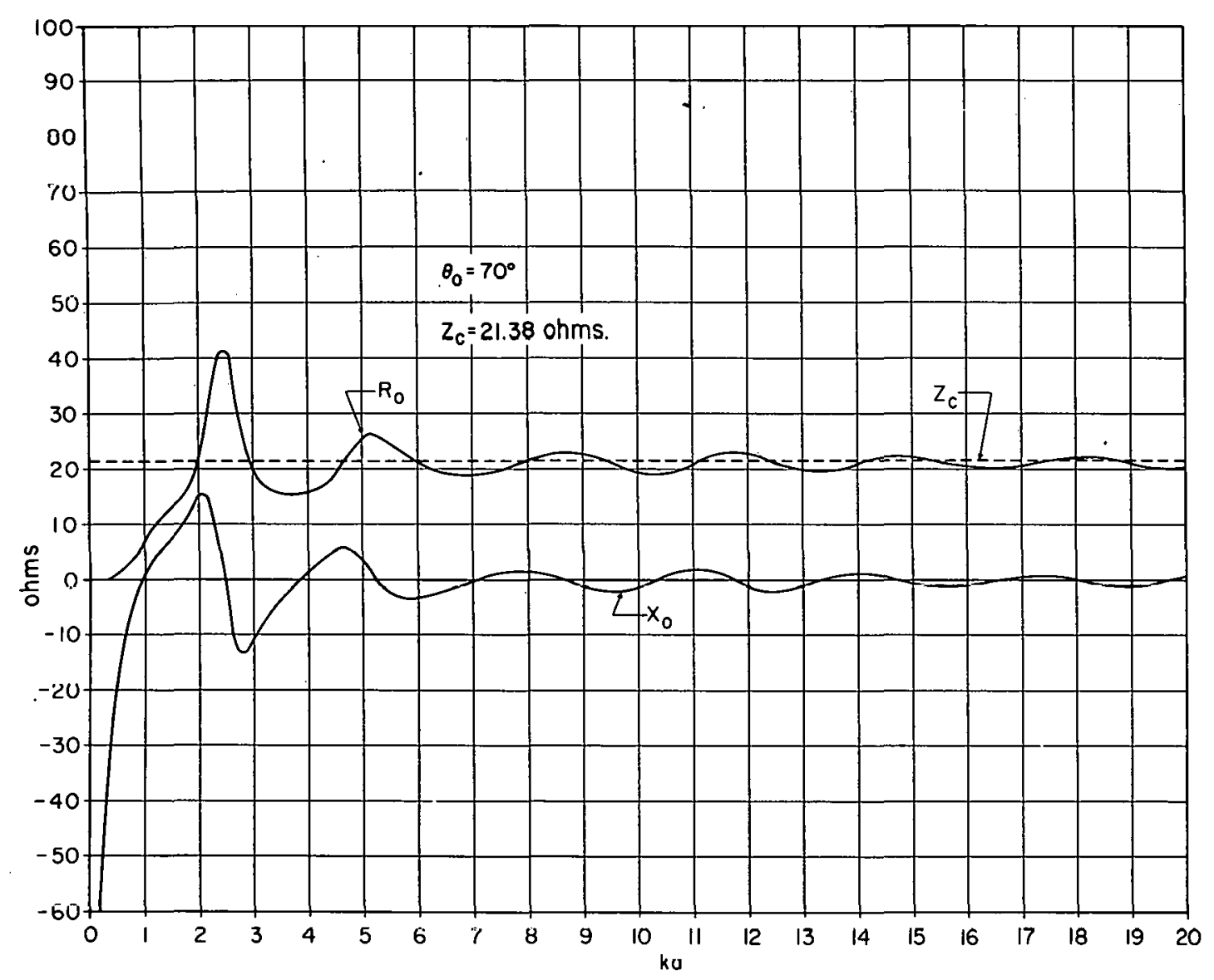

Fig. 21. Input Impedance $z_{0}=R_{0}+j x_{0}$ of Wide-Angle Conical Antennas 


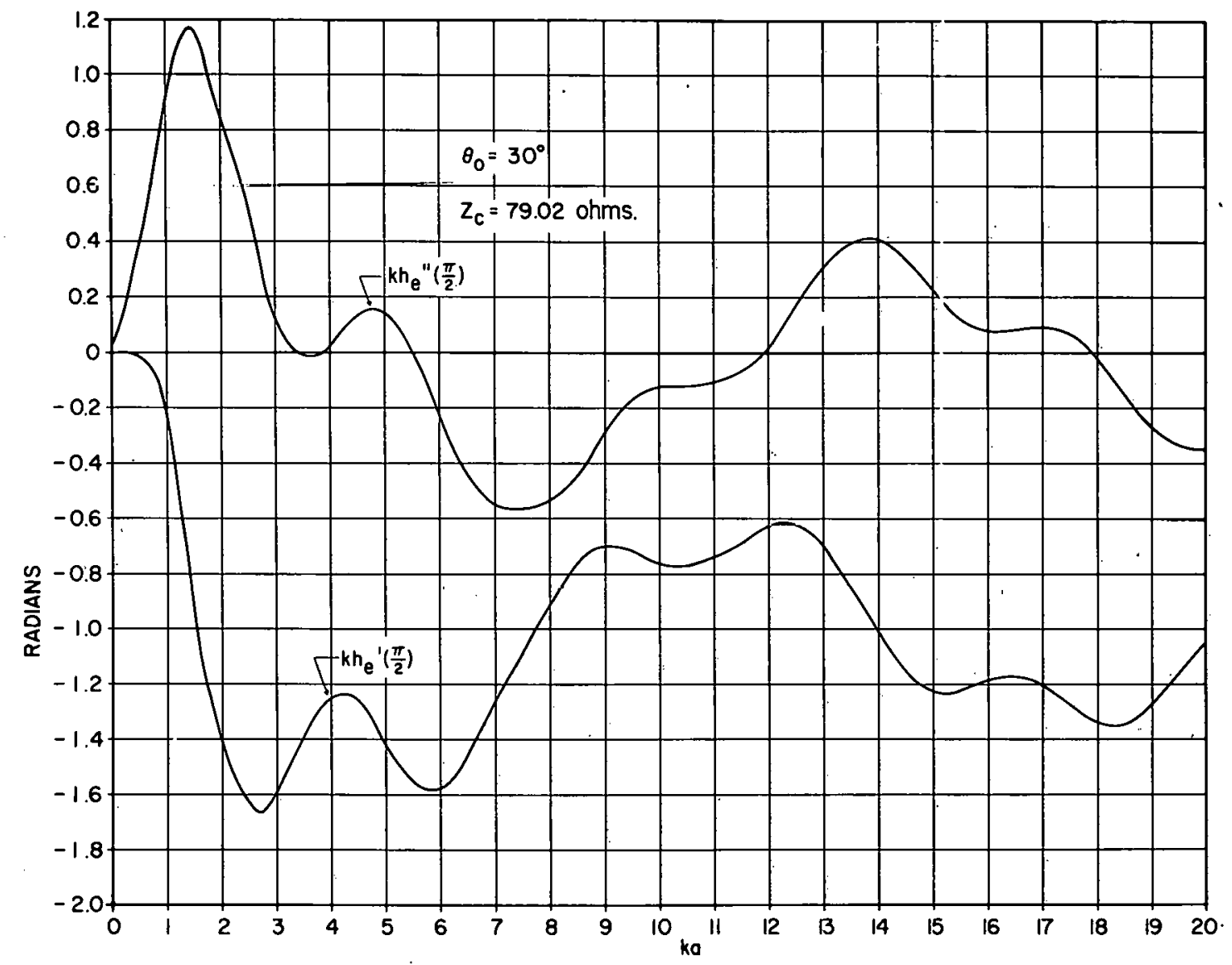

Fig. 22. Radian Effective Length $k h_{e}=k\left[h_{e}^{\prime \prime}+j h_{e}^{\prime}\right]$ of a Wide-Angle Conical Antenna 


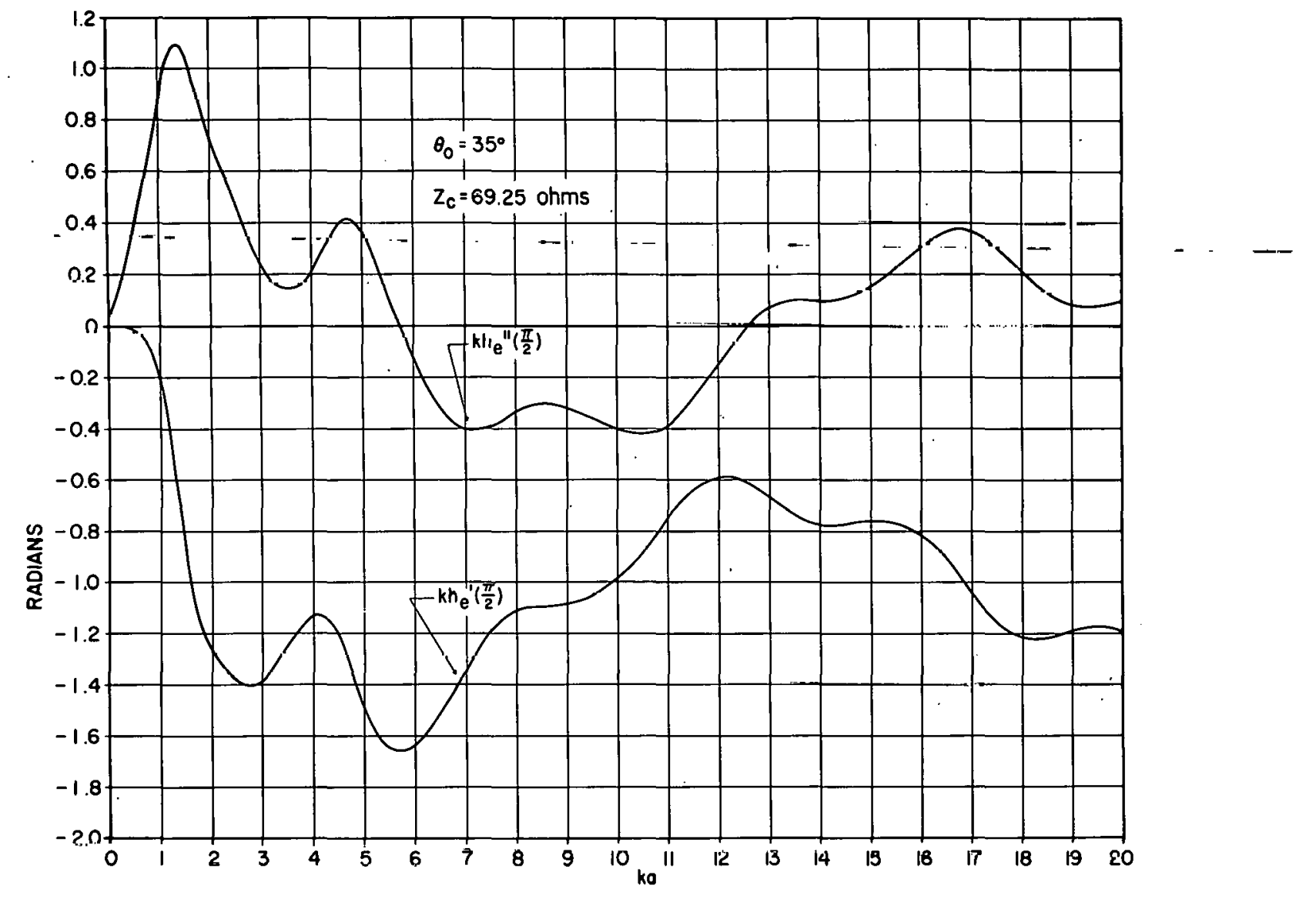

Fig. 23. Radian Effective Length $k h_{e}=k\left[h_{e}^{\prime \prime}+j h_{e}^{\prime}\right]$ of a Wide-Angle Conical Antenna 


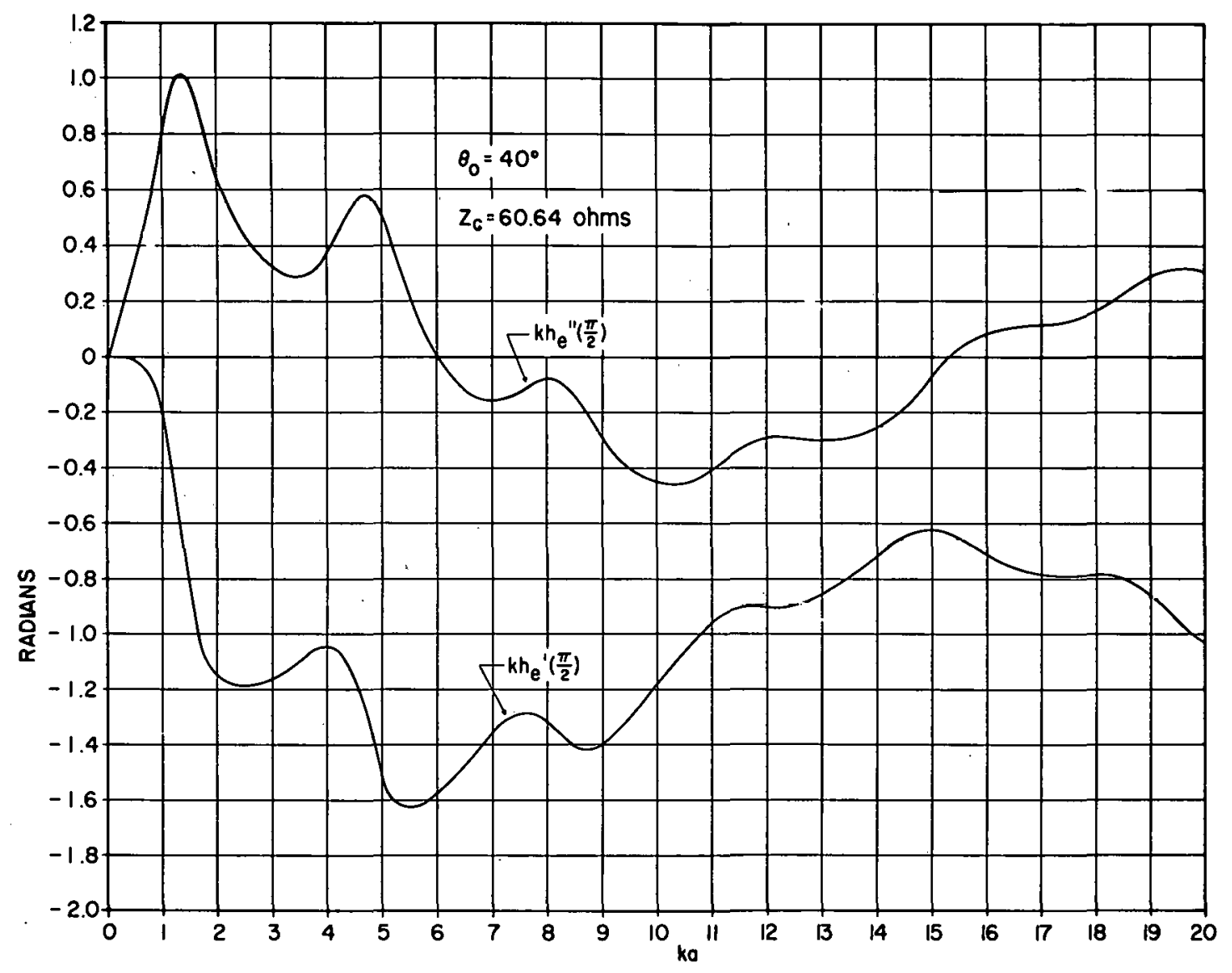

Fig. 24. Radian Effective Length $k h_{e}=k\left[h_{e}^{\prime \prime}+j h_{e}^{\prime}\right]$ of a Wide-Angle Conical Antenna 


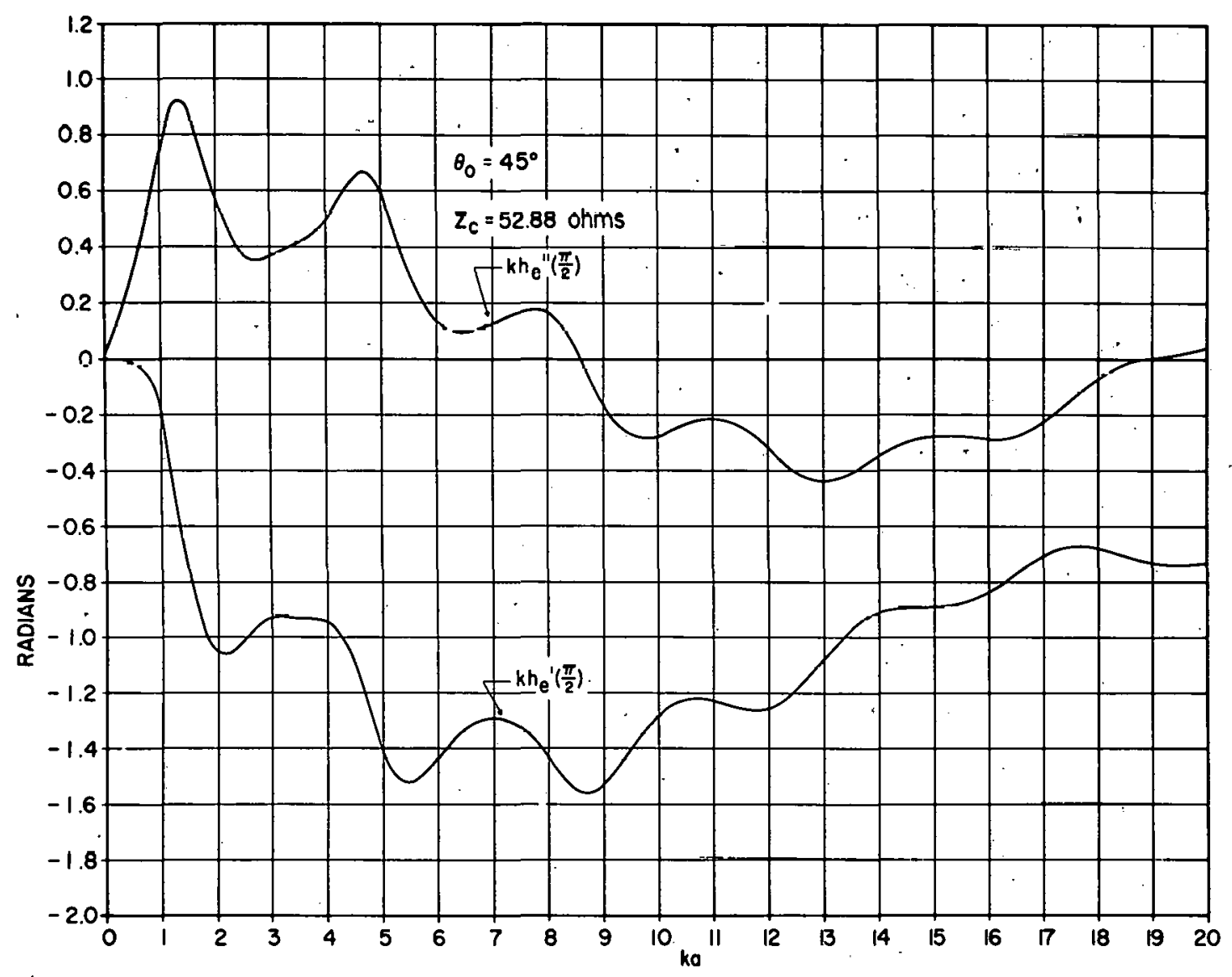

Fig. 25. Radian Effective Length $k h_{e}=k\left[h_{e}^{\prime \prime}+j h_{e}^{\prime}\right]$ of a Wide-Angle Conical Antenna 


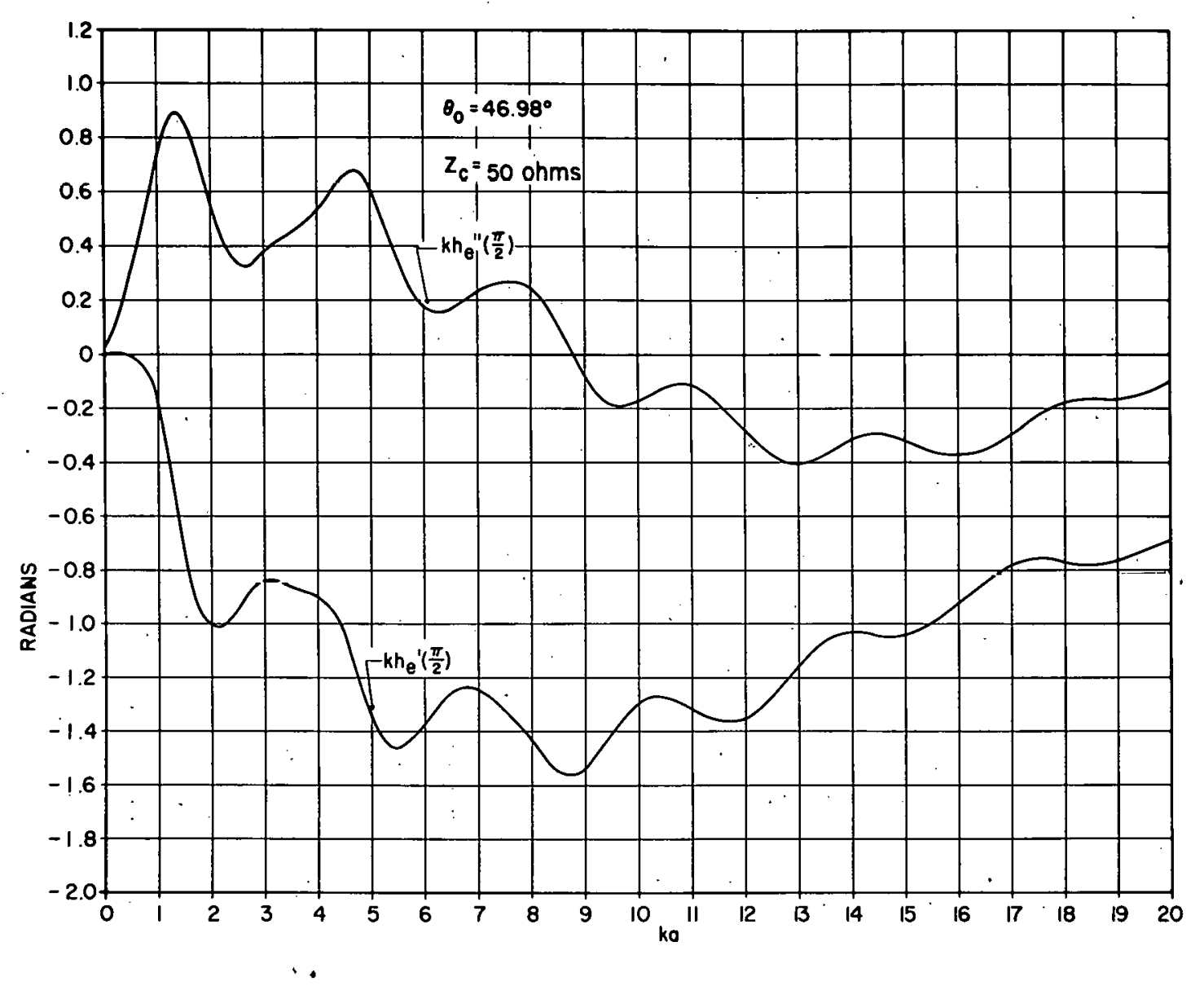

Fig. 26. Radian Effective Length $k_{e}=k\left[h_{e}^{\prime \prime}+j h_{e}^{\prime}\right]$ of a Wide-Angle Conical Antenna 


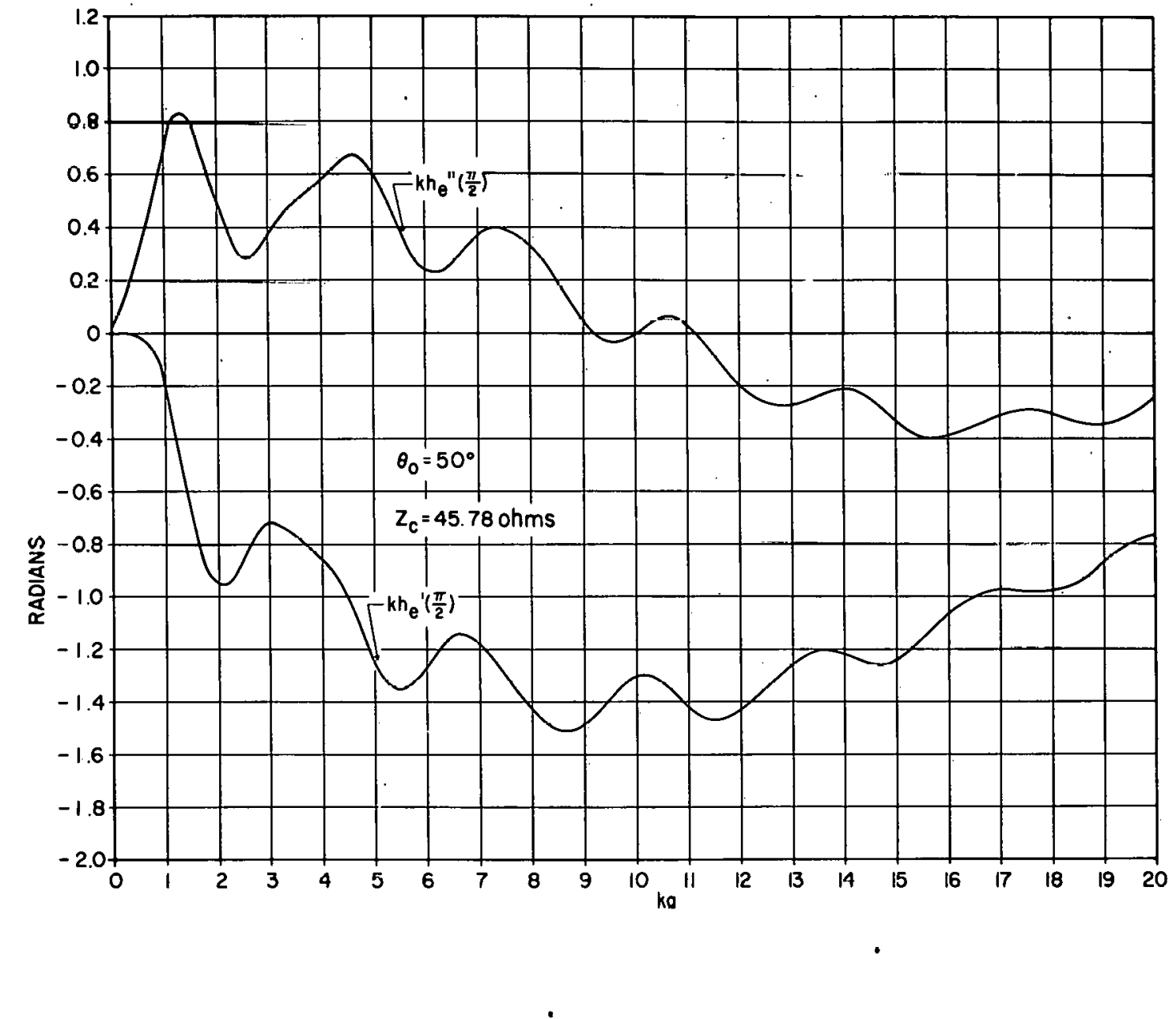

Fig. 27. Radian Effective Length $k h_{e}=k\left[h_{e}^{\prime \prime}+j h_{e}^{\prime}\right]$ of a Wide-Angle Conical Antenna 


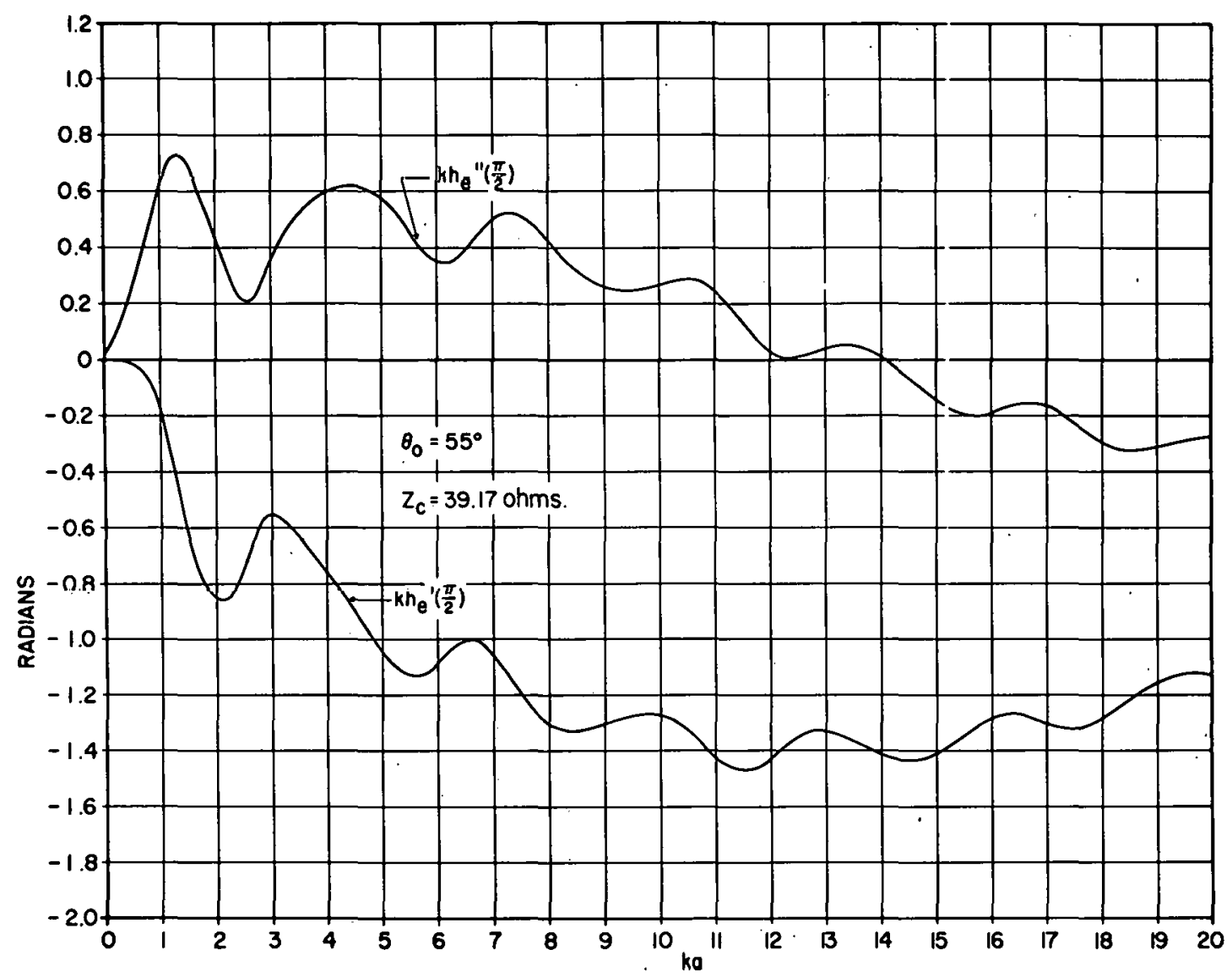

Fig. 28. Radian Effective Length $k h_{e}=k\left[h_{e}^{\prime \prime}+j h_{e}^{\prime}\right]$ of a Wide-Angle Conical Antenna 


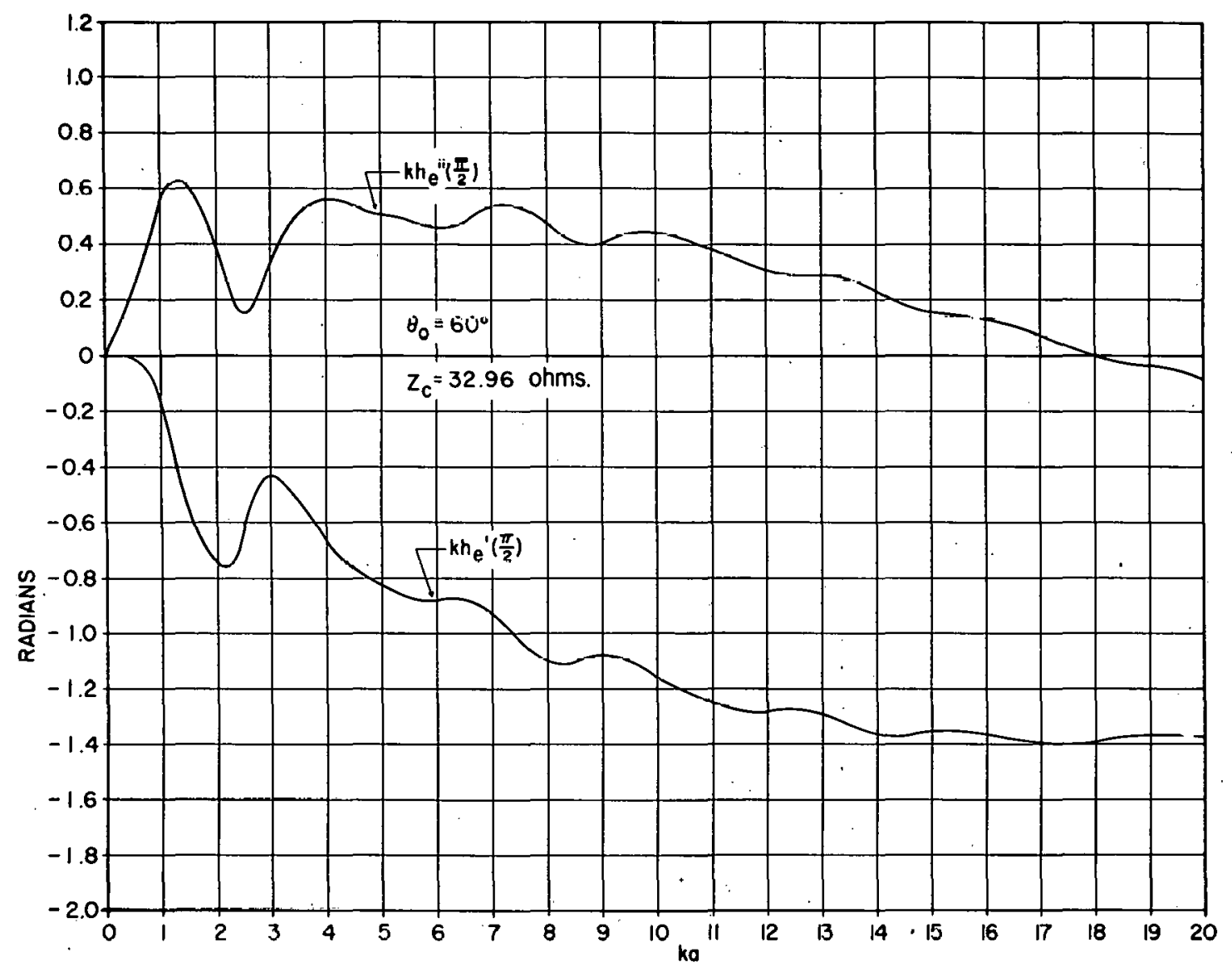

Fig. 29. Radian Effective Length $k h_{e}=k\left[h_{e}^{\prime \prime}+j h_{e}^{\prime}\right]$ of a Wide-Angle Conical Antenna 


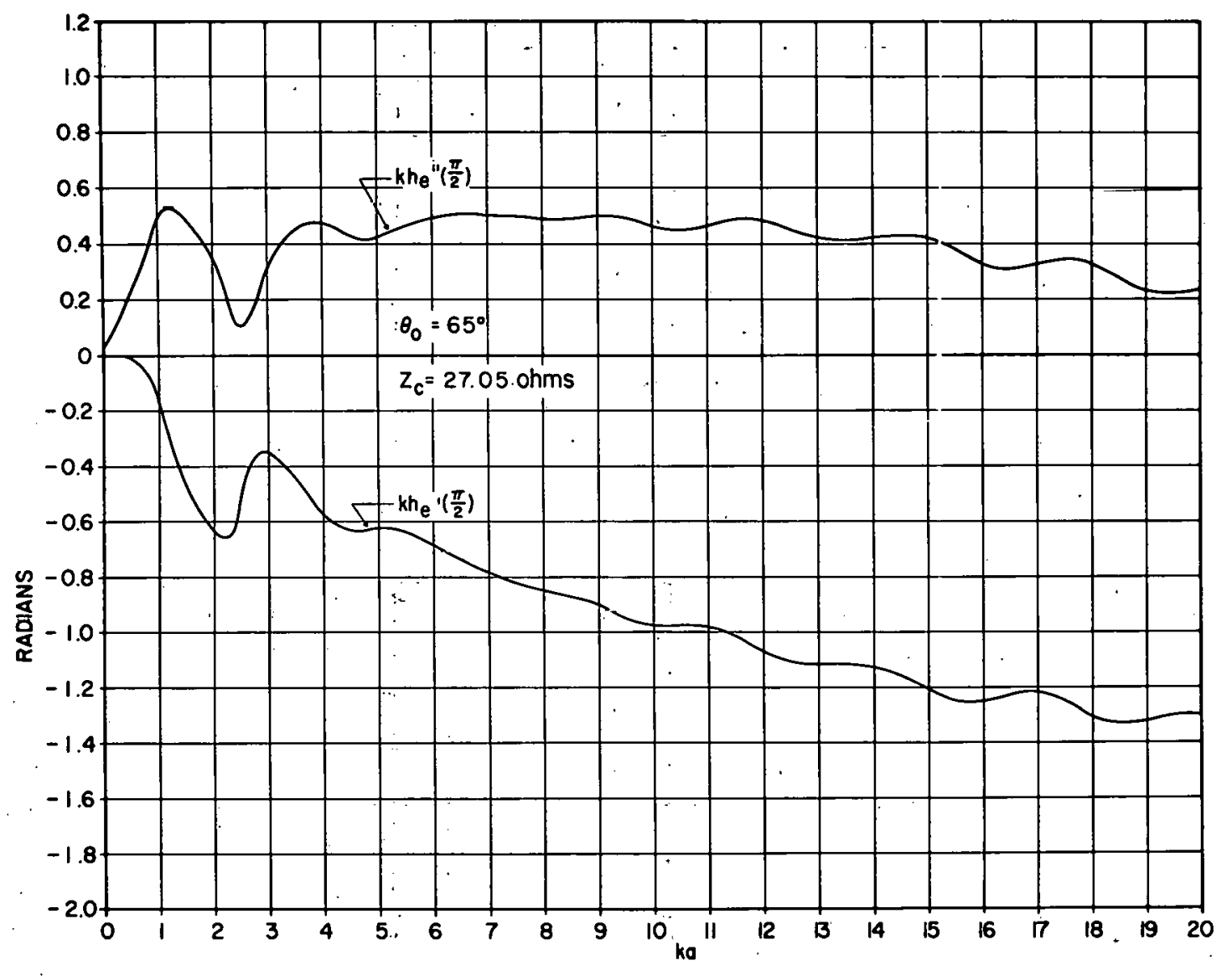

Fig. 30. Radian Effective Length $k h_{e}=k\left[h_{e}^{\prime \prime}+j h_{e}^{\prime}\right]$ of a Wide-Angle Conical Antenna 


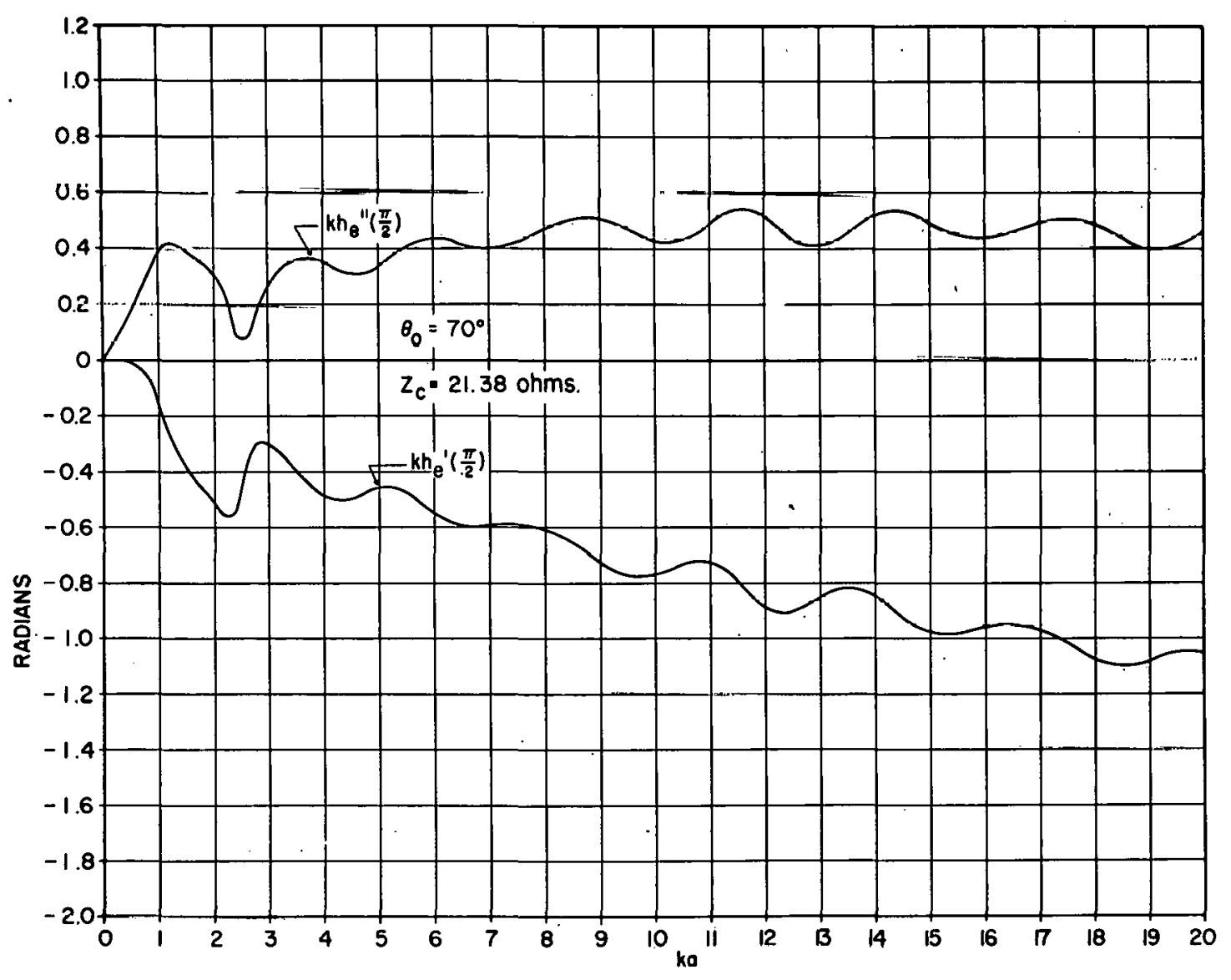

Fig. 31. Radian Effective Length $k h_{e}=k\left[h_{e}^{\prime \prime}+j h_{e}^{\prime}\right]$ of a Wide-Angle Conical Antenna 
(32a)
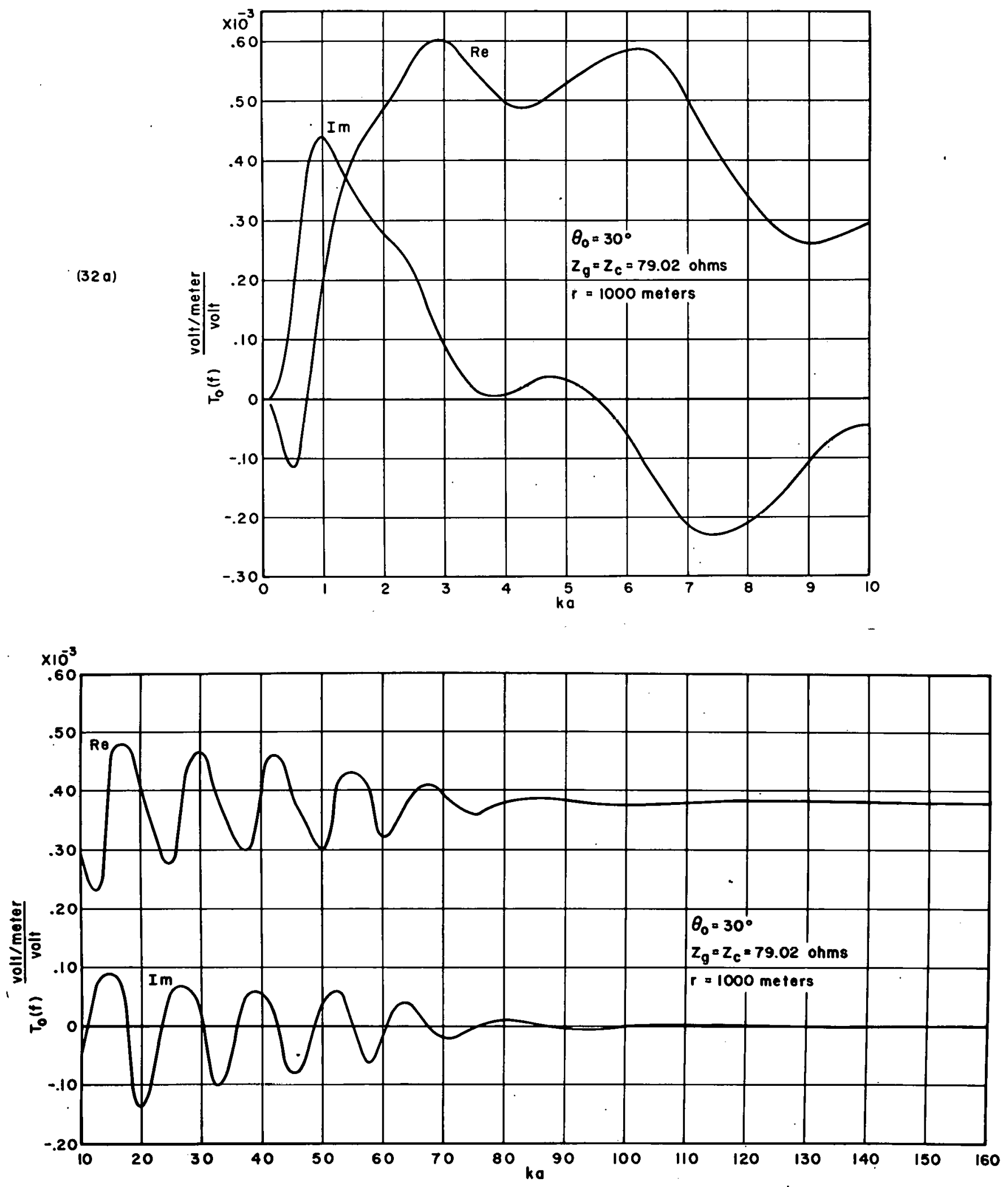

(32b)

Fig. 32a and Fig. 32b. Transmission Transfer Function of

Conical Antenna With Matched Source.

$\mathrm{E}^{\mathrm{rad}} / \mathrm{V}_{\mathrm{g}}=\mathrm{T}_{\mathrm{o}} \epsilon^{-j \mathrm{kr}}$ 

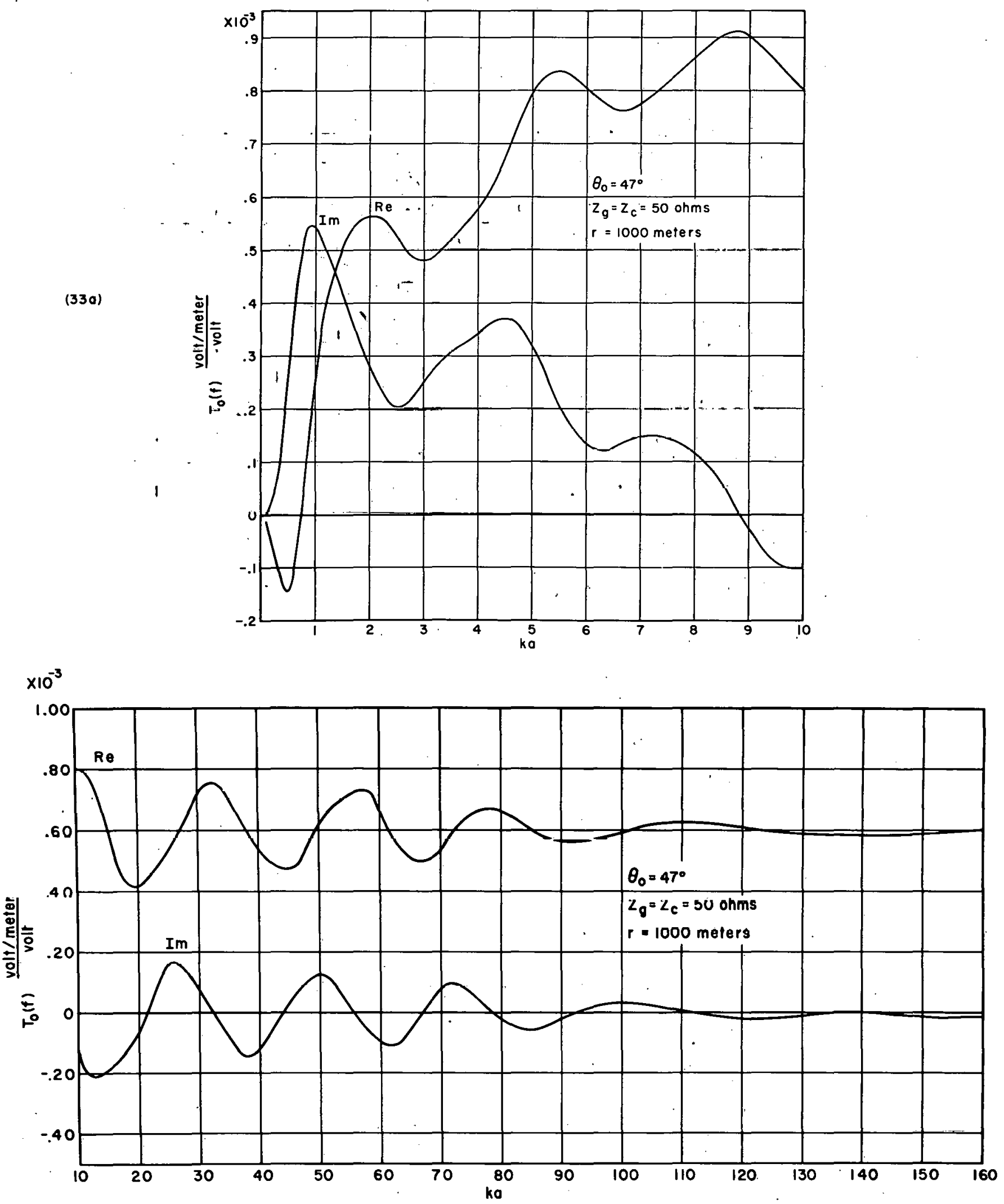

(33b)

Fig. 33a and Fig. 33b. Transmission Transfer Function of

Conical Antenna With Matched Source.

$\mathrm{E}^{\mathrm{rad}} / \mathrm{V}_{\mathrm{g}}=\mathrm{T}_{0} \epsilon^{-j k r}$ 


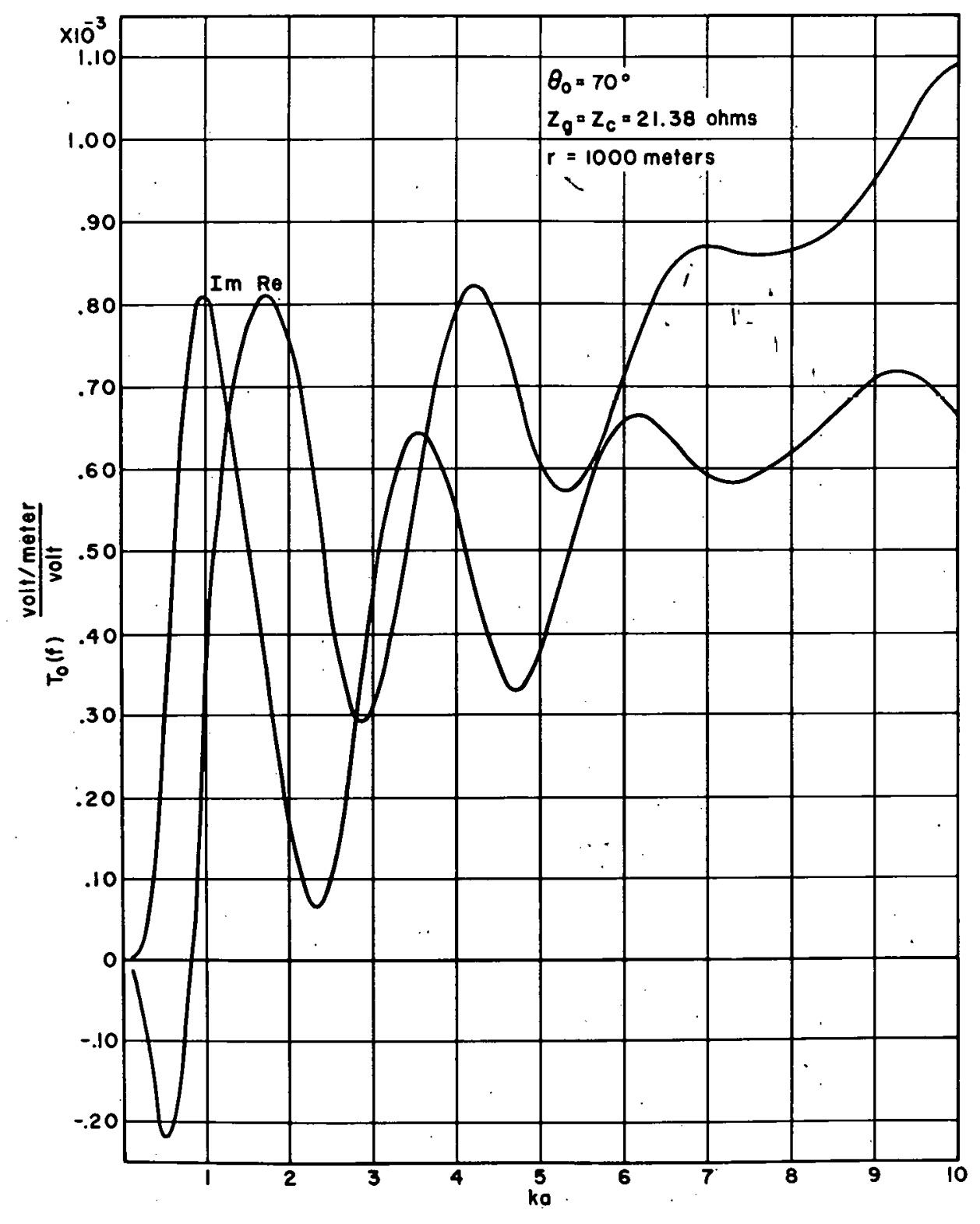

Fig. 34a. Transmission Transfer Function of Conical

Antenna With Matched Source. -

$\mathrm{E}^{-\dot{\mathrm{rad}}} / \mathrm{V}_{\mathrm{g}}=\mathrm{T}_{\mathrm{o}} \epsilon^{-j \mathrm{kr}}$ 


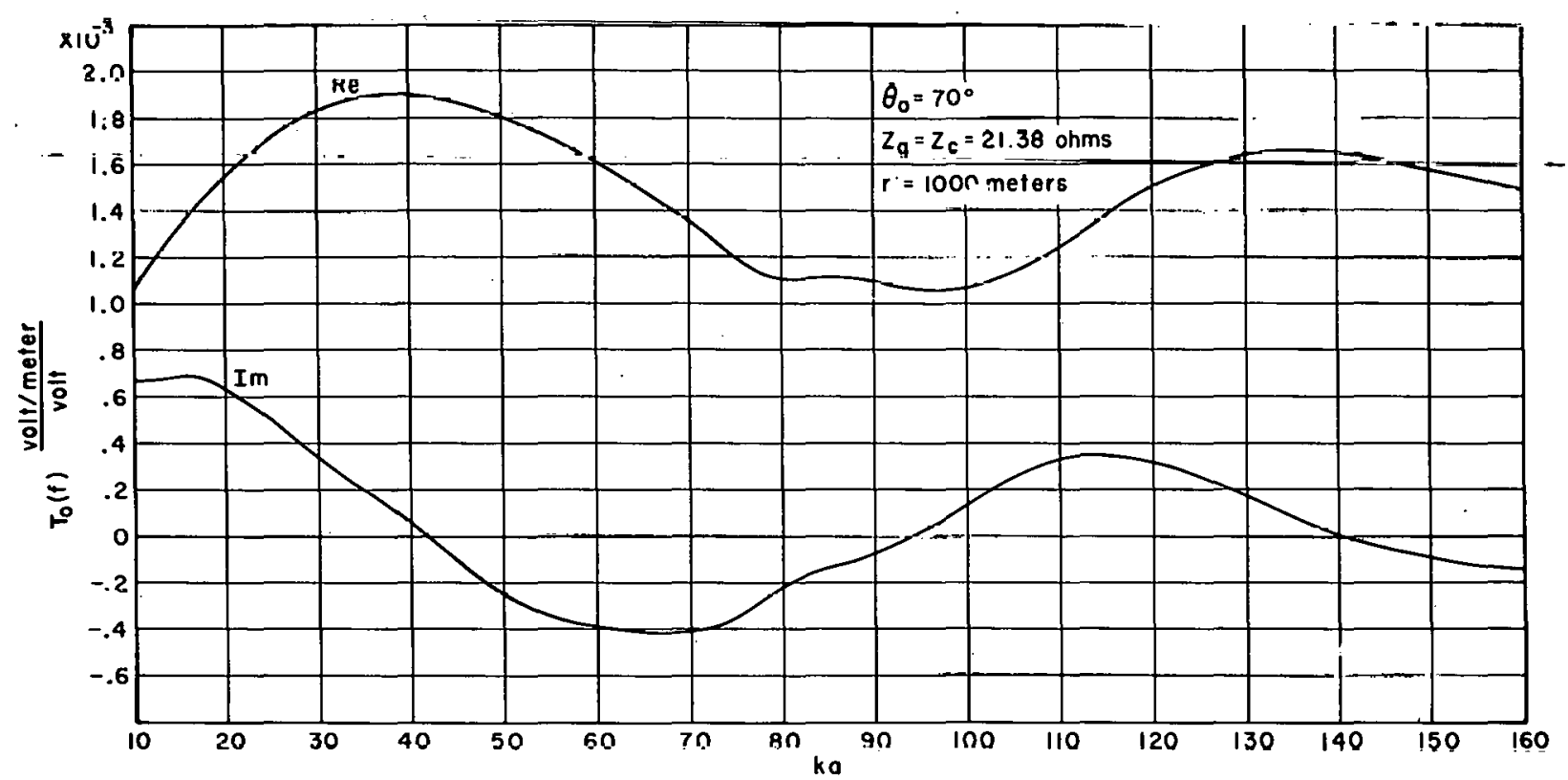

Fig. 34b. 'I'ransmission 'I'ransfer Finction of Conlcal Anterna With Matched Source.

$\mathrm{E}^{\mathrm{rad}} / \mathrm{V}_{\mathrm{g}}=\mathrm{T}_{\mathrm{o}} \epsilon^{-\mathrm{jkr}}$ 
(35a)

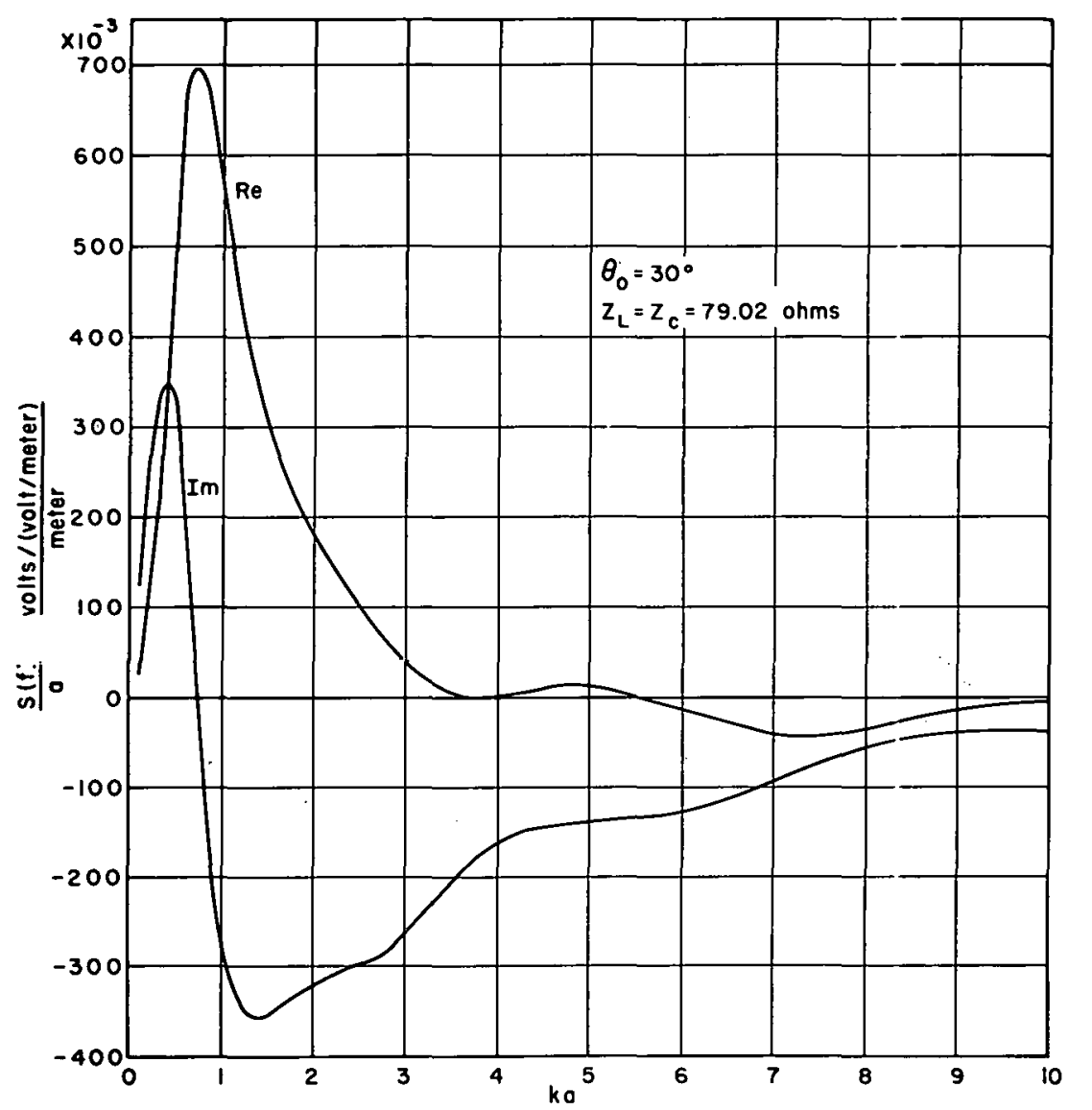

(35b)

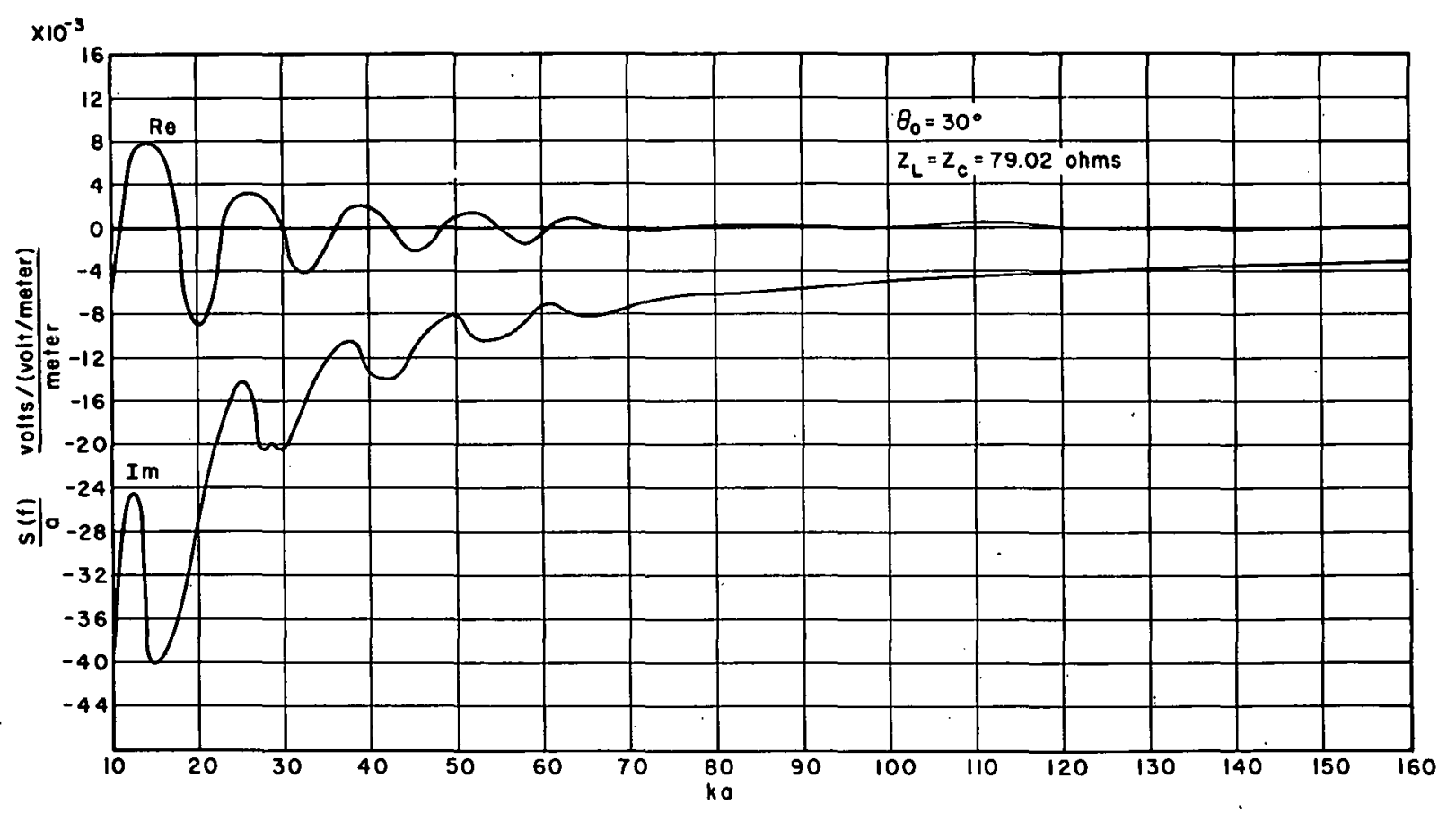

Fig. 35a and Fig. 35b. Reception Transfer Function per Unit of Antenna Length of Conical Antenna With Matched Load. $V_{L} / E^{i n c}=S$ 
(36 a)
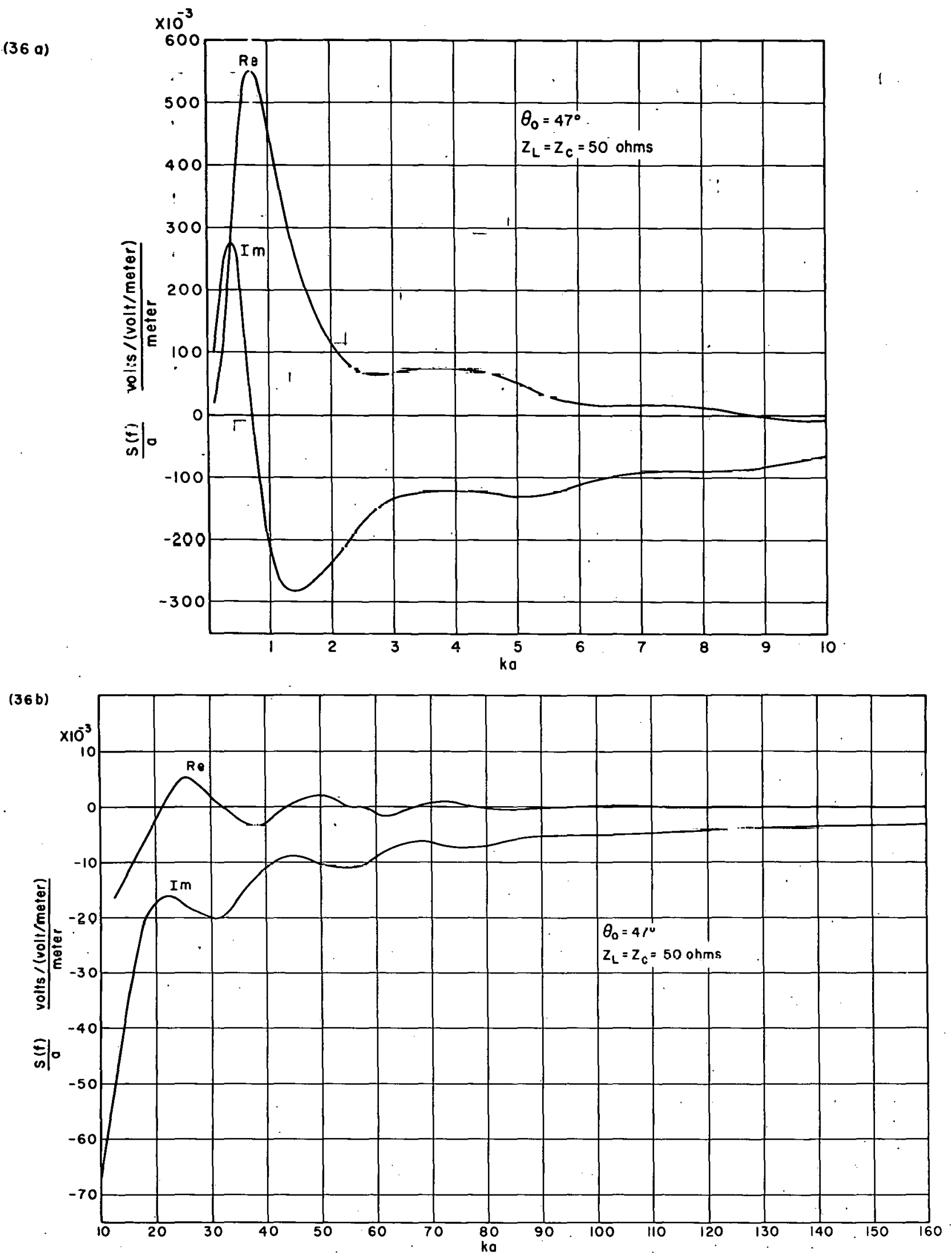

Fig. 36a and Fig. 36b. Reception Transfer Function per Unit of Antenna Length of . Conical Antenna With Matched Load. $V_{L} / E^{i n c}=* S$ 
(37a)

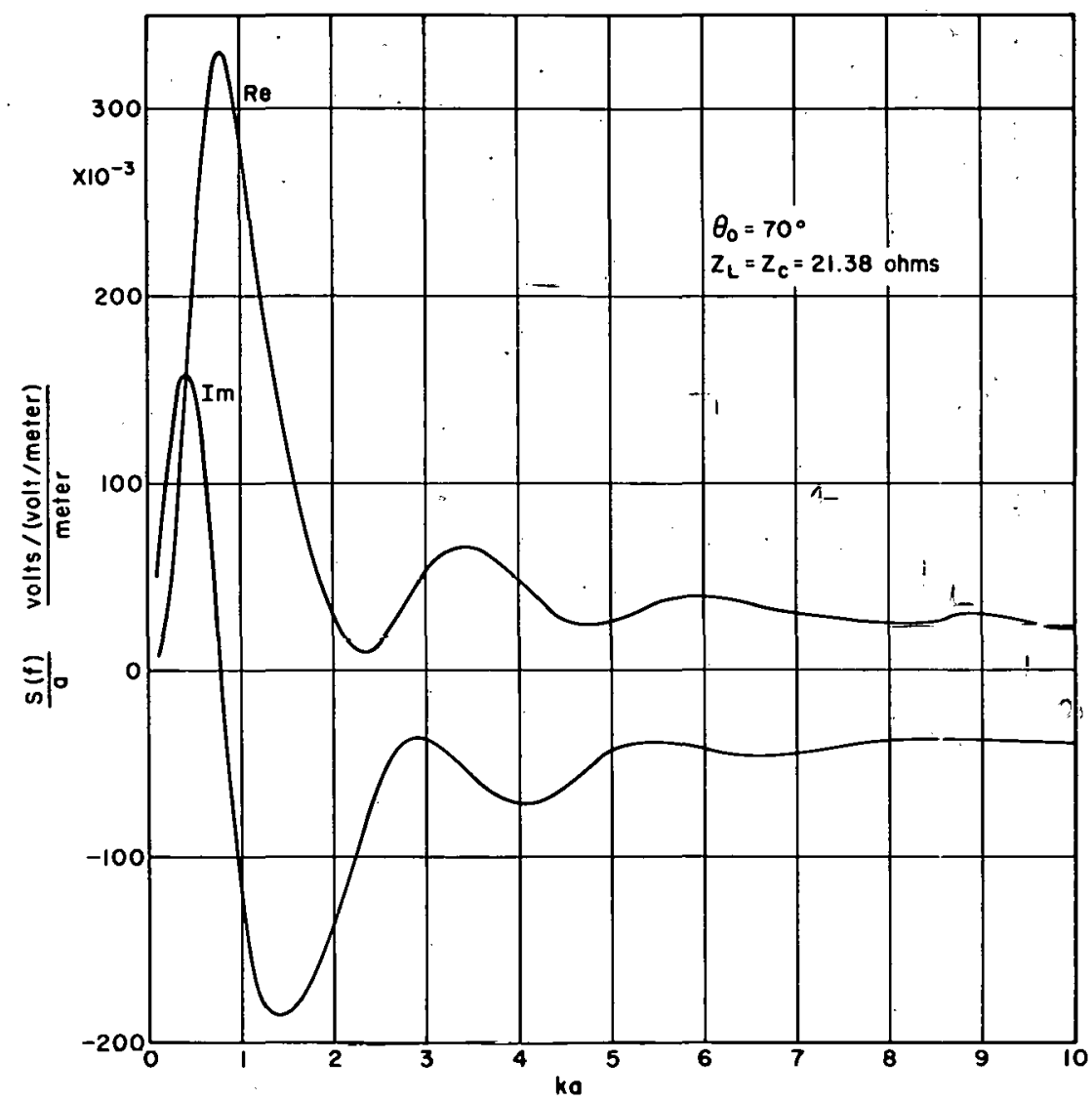

$(376)$

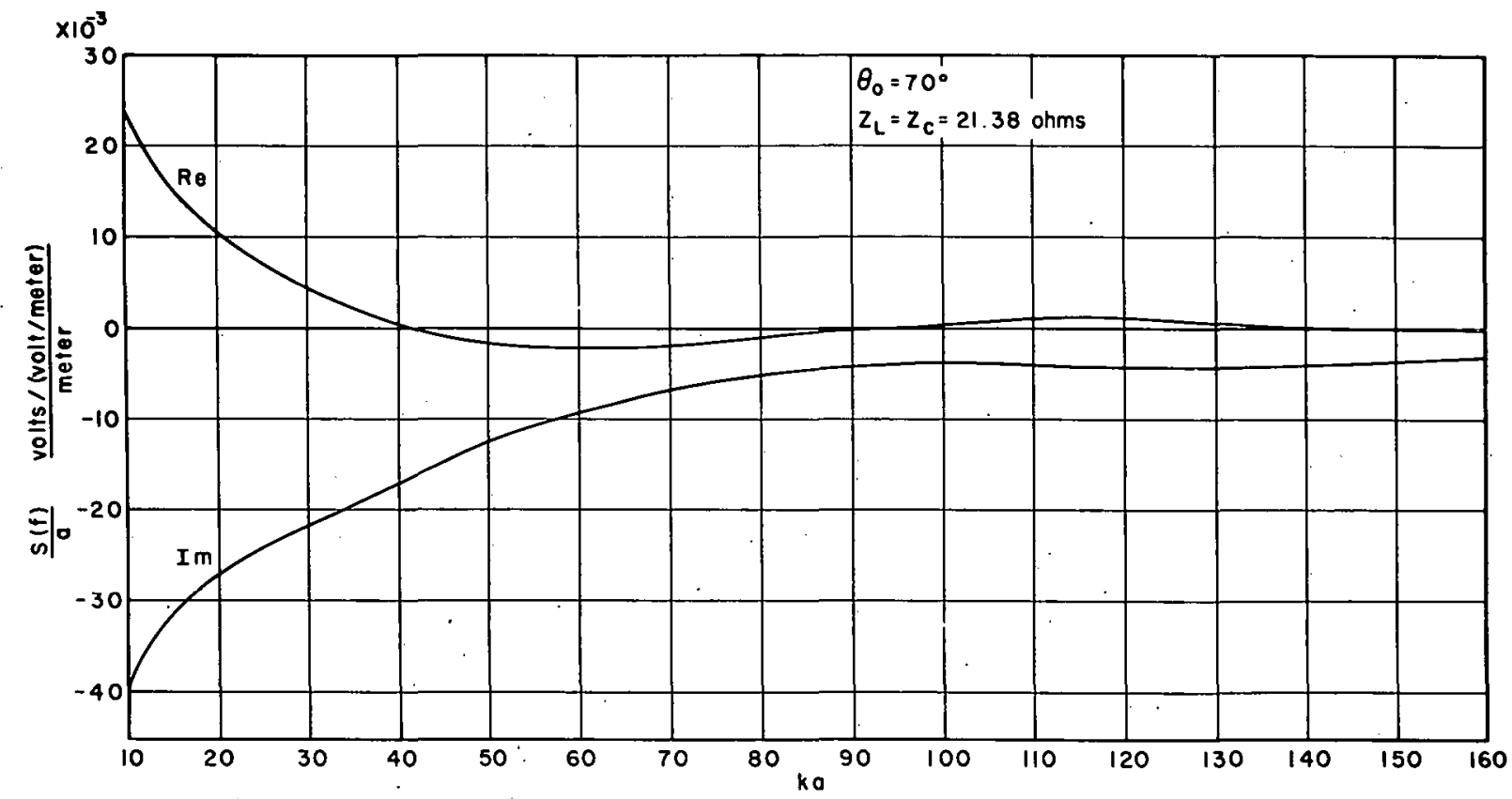

Fig. 37a and Fig. 37b. Reception Transfer Function per Unit of Antenna Length of. Conical Antenna With Matched Load. $V_{L} / E^{\text {inc }}=S$ 
1. Papas, C. H. and King, R. W. P., "Input Impedance of Wide-Angle Conical Antennas Fed by a Coaxial Line," Proc. IRE, 37, pp 1269-1271, Noverruer 1949.

2. Papas, C. H. and King, R. W. P., "Radiation From Wide-Angle Conical Antennas Fed by a Coaxial Line," Proc. IRE, 39 , pp.49-51, January 1951.

3. Harrison, C. W., Ir, , Tables of Impedance and Radian Effective Half-Length of Electrically Long Cylindrical Antennas of Fixed Length to Diameter Ratio, Sundia Corporation SCR-576, November 1962. A copy of this report may be obtälned 1rom the Tecluical Infurination De partment of Sonnia Corporation.

4. Harrison, C. W., Jr., "The Effective Half-Length of Cylindrical Antennas Less Than 1.3 Wavelengths Long," IFFE Trans. on Antennas and Propagation, AP-11, No. 6, pp 657-660, Púvenber 1063

5. Harrison, C. W. , Jr., Tables of Impedance and Radian Effective Length of Wide-Angle Cunical Antennas, Sandia Corporation SCR-662, June 1963. A copy of this report may be obtained from the Technical Information Department of Sandia Corporation.

6. Levine, H. and Papas, C. H., "Theory of the Circular Diffraction Antenna," J. of Appl. Phys. , 22 , No. 1, pp 29-43, January 1951.

7. King, R. W. P., Theory of Linear Antennas, Ch. 5, p. 570, Eq. (21), Harvard University Press, 1956.

8. Ibid, Ch. 2, p. 184, Eq. (6a).

9. Ibid, Ch. 4, p. 487, Eq. (21).

10. Williams, C.S., Jr., "The Reflected Vullage of a Line With an Antenna Load for Pulse Excltation," IEEE Transactions on Antennas and Propagation, AP-11, No. 3, pp 380-382, May 1963.

11. Erdélyi, A., et al. , Higher Transcendental.Functions, 2, McGraw-Hill Book Co., Inc., 1953, p. $183, \mathrm{Eq},(53)$.

12. Stratton, J. A., Electromagnetic Theory, McGraw-Hill Book Co., Inc., 1941, p. 404, Eq. (23).

13. McLaughlan, N. W., Bessel Functions for Engineers, 2nd Edition, Oxford Press, 1955, Formula 131, p. 198. 